\title{
LOW REGULARITY BLOWUP SOLUTIONS FOR THE MASS-CRITICAL NLS IN HIGHER DIMENSIONS
}

\author{
CHENMIN SUN AND JIQIANG ZHENG
}

\begin{abstract}
In this paper, we study the $H^{s}$-stability of the log-log blowup regime (which has been completely described in a series of recent works by Merle and Raphaël) for the focusing mass-critical nonlinear Schrödinger equations $i \partial_{t} u+\Delta u+|u|^{\frac{4}{d}} u=0$ in $\mathbb{R}^{d}$ with $d \geq 3$. We aim to extend the result in [Colliander and Raphael, Rough blowup solutions to the $L^{2}$ critical NLS, Math. Anna., 345(2009), 307-366.] for dimension two to the higher dimensions cases $d \geq 3$, where we use the bootstrap argument in the above paper and the commutator estimates in [M. Visan and X. Zhang, On the blowup for the $L^{2}$-critical focusing nonlinear Schrödinger equation in higher dimensions below the energy class. SIAM J. Math. Anal., 39(2007), 34-56. ].
\end{abstract}

Key Words: nonlinear Schrödinger equation; blow up, low regularity. AMS Classification: 35P25, 35Q55, 47J35.

\section{INTRODUCTION}

We study the initial-value problem for focusing nonlinear Schrödinger equations of the form

$$
\left\{\begin{array}{l}
\left(i \partial_{t}+\Delta\right) u=-|u|^{\frac{4}{d}} u, \quad(t, x) \in \mathbb{R} \times \mathbb{R}^{d}, \\
u(0, x)=u_{0}(x),
\end{array}\right.
$$

where $u: \mathbb{R}_{t} \times \mathbb{R}_{x}^{d} \rightarrow \mathbb{C}$.

Equation (1.1) admits a number of symmetries in $H^{1}\left(\mathbb{R}^{d}\right)$, explicitly:

- Space-time translation invariance: if $u(t, x)$ solves (1.1), then so does $u\left(t+t_{0}, x+x_{0}\right),\left(t_{0}, x_{0}\right) \in \mathbb{R} \times \mathbb{R}^{d} ;$

- Phase invariance: if $u(t, x)$ solves (1.1), then so does $e^{i \gamma} u(t, x), \gamma \in \mathbb{R}$;

- Galilean invariance: if $u(t, x)$ solves (1.1), then for $\beta \in \mathbb{R}^{d}$, so does $e^{i \frac{\beta}{2} \cdot\left(x-\frac{\beta}{2} t\right)} u(t, x-$ $\beta t)$;

- Scaling invariance: if $u(t, x)$ solves (1.1), then so does $u_{\lambda}(t, x)$ defined by

$$
u_{\lambda}(t, x)=\lambda^{\frac{d}{2}} u\left(\lambda^{2} t, \lambda x\right), \quad \lambda>0 .
$$

This scaling defines a notion of criticality for (1.1). In particular, one can check that the only homogeneous $L_{x}^{2}$-based Sobolev space that is left invariant under (1.2) is $L_{x}^{2}\left(\mathbb{R}^{d}\right)$, and we call problem (1.1) as mass-critical problem.

In above, we know that equation (1.1) admits a number of symmetries in the energy space $H^{1}$ : if $u(t, x)$ solves (1.1), then for any $\left(\lambda_{0}, t_{0}, x_{0}, \beta_{0}, \gamma_{0}\right) \in \mathbb{R}^{+} \times \mathbb{R} \times$ $\mathbb{R}^{d} \times \mathbb{R}^{d} \times \mathbb{R}$, so does

$$
v(t, x)=\lambda_{0}^{\frac{d}{2}} e^{i \gamma_{0}} e^{i \frac{\beta_{0}}{2}\left(x-\frac{\beta_{0}}{2} t\right)} u\left(\lambda_{0}^{2} t+t_{0}, \lambda_{0} x+x_{0}-\beta_{0} t\right) .
$$


From the Ehrenfest law or direct computation, these symmetries induce invariances in the energy space, namely

Mass: $M(u)=\int_{\mathbb{R}^{d}}|u(t, x)|^{2} d x=M\left(u_{0}\right) ;$
Energy: $E(u)=\int_{\mathbb{R}^{d}}\left(\frac{1}{2}|\nabla u(t, x)|^{2}-\frac{d}{2(d+2)}|u(t, x)|^{\frac{2(d+2)}{d}}\right) d x=E\left(u_{0}\right) ;$

Momentum: $P(u)=\operatorname{Im} \int_{\mathbb{R}^{d}} \nabla u \bar{u} d x=P\left(u_{0}\right)$.

In the focusing nonlinear Schrödinger equation, the special solutions play an important role. They are the so-called solitary waves and are of the form $u(t, x)=$ $e^{i w t} Q_{w}(x)$ (which is a global solution but not scatters), where $Q_{w}$ solves

$$
\left\{\begin{array}{l}
\Delta Q_{w}+Q_{w}\left|Q_{w}\right|^{\frac{4}{d}}=w Q_{w} \\
Q_{w} \in H^{1}\left(\mathbb{R}^{d}\right) \backslash\{0\}
\end{array}\right.
$$

Equation (1.7) is a standard nonlinear elliptic equation. It is known that if $w \leq 0$, then (1.7) does not have any solution. Therefore, we assume that $w>0$.

In dimension $d=1$, there exists a unique solution in $H^{1}$ up to translation to (1.7) and infinitely many with growing $L^{2}$-norm for $d \geq 2$. Nevertheless, from [1, 8, 14, there is a unique positive solution up to translation $Q_{w}(x) . Q_{w}$ is in addition radially symmetric. Letting $Q=Q_{w=1}$, then $Q_{w}(x)=w^{\frac{1}{p-1}} Q\left(w^{1 / 2} x\right)$ from scaling property, i.e. $Q$ solves

$$
\Delta Q+Q|Q|^{p-1}=Q .
$$

Using the Strichartz estimate and a standard fixed point argument, see Ginibre and Velo 9 and Cazenave and Weissler 2, we derive that (1.1) is locally wellposedness in $H^{s}$ for $0 \leq s \leq 1$ and the Cauchy problem is subcritical in $H^{s}$ for $s>0$ : for $u_{0} \in H^{s}, s>0$, there exists $0<T \leq+\infty$ such that $u \in C\left([0, T), H^{s}\right)$ and either $T=+\infty$ and we say the solution is global, or $T<+\infty$ and then

$$
\limsup _{t \rightarrow T}\|u(t)\|_{\dot{H}^{s}}=+\infty
$$

and we say the solution blows up in finite time.

In the case $\left\|u_{0}\right\|_{L^{2}}<\|Q\|_{L^{2}}$, from classical variational arguments, one can obtain the global well-posedness in $H^{1}\left(\mathbb{R}^{d}\right)$. Indeed, this follows from the conservation of the energy, the mass and Gagliardo-Nirenberg inequality as exhibited by Weinstein in [28]:

$$
\forall u \in H^{1}: E(u) \geq \frac{1}{2}\left(\int|\nabla u|^{2}\right)\left(1-\left(\frac{\int|u|^{2}}{\int Q^{2}}\right)\right) .
$$

Moreover, the scattering result in $L^{2}\left(\mathbb{R}^{d}\right)$ is obtained by Killip, Tao, Visan and Zhang [12, 13] for radial initial data and Dodson [6] for nonradial initial data.

In the case $\left\|u_{0}\right\|_{L^{2}}=\|Q\|_{L^{2}}$, the pseudo-conformal transformation applied to the stationary solution $e^{i t} Q$ yields an explicit solution

$$
S(t, x)=\frac{1}{|t|^{d / 2}} Q\left(\frac{x}{t}\right) e^{-i \frac{|x|^{2}}{4 t}+\frac{i}{t}},\|S(t)\|_{L^{2}}=\|Q\|_{L^{2}}
$$

which scatters as $t \rightarrow-\infty$ in the sense that there exists $\psi_{-} \in L^{2}\left(\mathbb{R}^{d}\right)$ such that

$$
\lim _{t \rightarrow-\infty}\left\|S(t, x)-e^{i t \Delta} \psi_{-}\right\|_{L^{2}\left(\mathbb{R}^{d}\right)}=0 .
$$


And $S(t, x)$ blows up at $T=0$ at the speed

$$
\|\nabla S(t)\|_{L^{2}} \sim \frac{1}{|t|} .
$$

An essential feature of (1.10) is compact up to the symmetries of the flow, meaning that all the mass goes into the singularity formation

$$
|S(t)|^{2} \rightarrow\|Q\|_{L^{2}}^{2} \delta_{x=0} \text { as } t \rightarrow 0 .
$$

It turns out that $S(t)$ is the unique minimal mass blow-up solution in $H^{1}$ in the following sense: let $u(-1) \in H^{1}$ with $\|u(-1)\|_{L^{2}}=\|Q\|_{L^{2}}$, and assume that $u(t)$ blows up at $T=0$, then $u(t)=S(t)$ up to the symmetries of the equation, see Merle [15] (radial) and [16] (general case). Note that from direct computation

$$
E(S(t, x))>0, \text { and }\|\nabla S(t)\|_{L^{2}}=\frac{C}{|t|} .
$$

The general intuition is that such a behavior is exceptional in the sense that such minimal elements can be classified.

The situation $\left\|u_{0}\right\|_{L^{2}}>\|Q\|_{L^{2}}$ has been clarified by Merle and Raphäl in the series of papers $17,18,19,20,21,25$. Let us define the differential operator

$$
\Lambda:=\frac{d}{2}+y \cdot \nabla
$$

which will be of constant use. Then we introduce the following property:

Spectral property. Let $d \geq 1$. Consider the two real Schrödinger operators

$$
\mathcal{L}_{1}=-\Delta+\frac{2}{d}\left(\frac{4}{d}+1\right) Q^{\frac{4}{d}-1} y \cdot \nabla Q, \mathcal{L}_{2}=-\Delta+\frac{2}{d} Q^{\frac{4}{d}-1} y \cdot \nabla Q,
$$

and the real quadratic form for $\varepsilon=\varepsilon_{1}+i \varepsilon_{2} \in H^{1}$ :

$$
H(\varepsilon, \varepsilon)=\left(\mathcal{L}_{1} \varepsilon_{1}, \varepsilon_{1}\right)+\left(\mathcal{L}_{2} \varepsilon_{2}, \varepsilon_{2}\right) .
$$

Then there exists a universal constant $\tilde{\delta}_{1}>0$ such that for all $\varepsilon \in H^{1}$, if

$$
\left(\varepsilon_{1}, Q\right)=\left(\varepsilon_{1}, Q_{1}\right)=\left(\varepsilon_{1}, y Q\right)=\left(\varepsilon_{2}, Q_{1}\right)=\left(\varepsilon_{2}, Q_{2}\right)=\left(\varepsilon_{2}, \nabla Q\right)=0,
$$

then

$$
H(\varepsilon, \varepsilon) \geq \tilde{\delta}_{1} \int\left(|\nabla \varepsilon|^{2}+|\varepsilon|^{2} e^{-(2-)|y|}\right)
$$

where $Q_{1}=\Lambda Q$ and $Q_{2}=\Lambda Q_{1}$, and $2_{-}=2-\epsilon$ with $0<\epsilon \ll 1$.

Remark 1.1. We remark that this spectral property has been proved rigorously in 17] for dimension $d=1$, since the ground state $Q$ is explicit in dimension one and the spectral property could be deduced from some known properties of the secondorder differential operators [26]. For the dimensions $d \in\{2,3,4\}$, Fibich, Merle and Raphael[7] gave a numerically-assisted proof of the above spectral property by using the numerical representation of the ground state $Q$. Rectently, Yang, Roudenko and Zhao 30 showed the spectral property in dimensions $5 \leq d \leq 10$ (general case) and $d \in\{11,12\}$ (radial).

Based on the works 17, 18, 19, 20, 21, 25, we now have: 
Theorem 1.2 (Dynamics of NLS). Let $d \geq 1$ and assume the spectral property holds true. Then there exists $\alpha^{*}>0$ and a universal constant $C^{*}>0$ such that the following is true. Let $u_{0} \in \mathcal{B}_{\alpha^{*}}$ with

$$
\mathcal{B}_{\alpha^{*}}:=\left\{u_{0} \in H^{1}\left(\mathbb{R}^{d}\right): \int Q^{2} \leq \int\left|u_{0}\right|^{2}<\int Q^{2}+\alpha^{*}\right\},
$$

let $u(t)$ be the corresponding solution to (1.1) with $[0, T)$ its maximum time interval with existence on the right in $H^{1}$.

(i) Estimates on the blow-up speed: assume $u(t)$ blows up in finite time i.e., $0<T<+\infty$, for $t$ close enough to $T$, we have either

$$
\lim _{t \rightarrow T} \frac{\|\nabla u\|_{L_{x}^{2}}}{\|Q\|_{L_{x}^{2}}}\left(\frac{T-t}{\ln |\ln (T-t)|}\right)^{\frac{1}{2}}=\frac{1}{\sqrt{2 \pi}}
$$

or

$$
\|\nabla u(t)\|_{L^{2}} \geq \frac{C^{*}}{(T-t) \sqrt{E^{G}\left(u_{0}\right)}}
$$

with

$$
E^{G}(u):=E(u)-\frac{1}{2} \frac{P(u)^{2}}{\|u\|_{L_{x}^{2}}^{2}} .
$$

(ii) Description of the singularity: assume $u(t)$ blows up in finite time, then there exist parameters $(\lambda(t), x(t), \gamma(t)) \in \mathbb{R}^{+} \times \mathbb{R}^{d} \times \mathbb{R}$ and an asymptotic profile $u^{*} \in L^{2}\left(\mathbb{R}^{d}\right)$ such that

$$
u(t)-\frac{1}{\lambda(t)^{d / 2}} Q\left(\frac{x-x(t)}{\lambda(t)}\right) e^{i \gamma(t)} \rightarrow u^{*} \quad \text { in } \quad L^{2}\left(\mathbb{R}^{d}\right) \quad \text { as } \quad t \rightarrow T .
$$

Moreover, the blow up point is finite in the sense that

$$
x(t) \rightarrow x(T) \in \mathbb{R}^{d}, \quad \text { as } t \rightarrow T .
$$

Moreover, assume $u(t)$ satisfies (1.13), $x(T)$ be its blow up point. Set

$$
\lambda_{0}(t)=\sqrt{2 \pi} \sqrt{\frac{T-t}{\ln |\ln (T-t)|}}
$$

then there exists a phase parameter $\gamma_{0}(t) \in \mathbb{R}$ such that:

$$
u(t)-\frac{1}{\lambda_{0}(t)^{d / 2}} Q\left(\frac{x-x_{0}(T)}{\lambda(t)}\right) e^{i \gamma_{0}(t)} \rightarrow u^{*} \quad \text { in } \quad L^{2}\left(\mathbb{R}^{d}\right) \quad \text { as } t \rightarrow T .
$$

(iii) Universality of blow up profile in $\dot{H}^{1}$ : if we assume that $u(t)$ blows up in finite time with (1.13), then there exist parameters $\lambda_{0}(t)=\frac{\|\nabla Q\|_{L_{x}^{2}}}{\|\nabla u(t)\|_{L_{x}^{2}}}, x_{0}(t) \in \mathbb{R}^{d}$ and $\gamma_{0}(t) \in \mathbb{R}$ such that

$$
e^{i \gamma_{0}(t)} \lambda_{0}(t)^{\frac{d}{2}} u\left(t, \lambda_{0}(t) x+x_{0}(t)\right) \rightarrow Q \quad \text { in } \quad \dot{H}^{1}, \quad \text { as } \quad t \rightarrow T .
$$

If $u(t)$ satisfies (1.14), then asymptotic stability (1.18) holds on a sequence $t_{n} \rightarrow T$.

(iv) Sufficient condition for log-log blow-up: if

$$
E^{G}(u):=E(u)-\frac{1}{2} \frac{P(u)^{2}}{\|u\|_{L_{x}^{2}}^{2}}<0,
$$

then $u(t)$ blows up in finite time with the log-log speed (1.13). More generally, the set of initial data $u_{0} \in \mathcal{B}_{\alpha^{*}}$ such that the corresponding solution $u(t)$ to (1.1) blows 
up in finite time $0<T<+\infty$ with the log-log speed (1.13) is open in $H^{1}\left(H^{1}\right.$ stability of the log-log regime).

$(v)$ Asymptotic of $u^{*}$ on the singularity: assume $T<+\infty$; if $u(t)$ satisfies (1.13), then for $R>0$ small,

$$
\frac{1}{C^{*}(\ln |\ln (R)|)^{2}} \leq \int_{|x-x(T)|<R}\left|u^{*}(x)\right|^{2} d x \leq \frac{C^{*}}{(\ln |\ln (R)|)^{2}}
$$

which implies $u^{*} \notin H^{1}$ and $u^{*} \notin L^{p}$ with $p>2$. If $u(t)$ satisfies (1.14), then

$$
\int_{|x-x(T)| \leq R}\left|u^{*}(x)\right|^{2} d x \leq C^{*} E_{0} R^{2} \text {, and } u^{*} \in H^{1} .
$$

Remark 1.3. The above theorem asserts the existence and the stability of a log$\log$ blowup regime, and gives sufficient conditions to ensure its occurrence. It also assets that the log-log blowup regime is open in $H^{1}$. In [5], Colliander and Raphael proved that the log-log blowwup dynamics described by Theorem 1.2 are stable under small $H^{s}$ perturbations with $0<s \leq 1$ in dimension $d=2$. In this paper, we extend their result to higher dimensional cases $d \geq 3$.

Now, we state our main result.

Theorem 1.4 ( $H^{s}$-stability of the $\log$-log regime). Let $d \geq 3$ and $\frac{1}{1+\min \left\{1, \frac{4}{d}\right\}}<s \leq$ 1 , and assume the spectral property holds true. Then, the log-log blowup dynamics described by Theorem 1.2 are stable under small $H^{s}$ perturbations. In other words, let $u_{0} \in H^{1}$ evolve into a log-log blowup solution given by Theorem 1.2. Then, there exists $\varepsilon=\varepsilon\left(s, u_{0}\right)$ such that for any $v_{0} \in H^{s}$ with

$$
\left\|v_{0}-u_{0}\right\|_{H^{s}\left(\mathbb{R}^{d}\right)}<\varepsilon
$$

then, the corresponding solution $v(t)$ to (1.1) blows up in finite time $0<T<+\infty$ with the following blowup dynamics: there exist geometrical parameters $(\lambda(t), x(t), \gamma(t)) \in$ $(0,+\infty) \times \mathbb{R}^{d} \times \mathbb{R}$ and an asymptotic residual profile $v^{*} \in L^{2}$, with $v^{*} \notin L^{p}(p>2)$, such that as $t \rightarrow T$

$$
\begin{array}{r}
v(t)-\frac{1}{\lambda(t)^{\frac{d}{2}}} Q\left(\frac{x-x(t)}{\lambda(t)}\right) e^{i \gamma(t)} \rightarrow v^{*} \quad \text { in } L^{2}, \\
x(t) \rightarrow x(T) \in \mathbb{R}^{d}, \\
\lambda(t) \sqrt{\frac{\log |\log (T-t)|}{T-t}} \rightarrow \sqrt{2 \pi} .
\end{array}
$$

Remark 1.5. The restriction $\frac{1}{1+\min \left\{1, \frac{4}{d}\right\}}<s$ follows from the commutator estimation in Lemma 2.5.

1.1. Outline of proof of Theorem[1.4. First, we recall from [18, 19] the existence of a one parameter family of localized self-similar profiles close to the ground state solution $Q$.

Proposition 1.6 (Localized self-similar profiles, [18, 19]). There exist universal constants $C>0, \eta^{*}>0$ such that the following holds true. For all $0<\eta<\eta^{*}$, there exist constants $\varepsilon^{*}(\eta)>0, b^{*}(\eta)>0$ going to zero as $\eta \rightarrow 0$ such that for all 
$|b|<b^{*}(\eta)$, there exists a unique radial solution $\tilde{Q}_{b}$ to

$$
\left\{\begin{array}{l}
\Delta \tilde{Q}_{b}-\tilde{Q}_{b}+i b \Lambda \tilde{Q}_{b}+\tilde{Q}_{b}\left|\tilde{Q}_{b}\right|^{\frac{4}{d}}=0 \\
P_{b}=\tilde{Q}_{b} e^{i \frac{b|y|^{2}}{4}}>0 \text { in } B_{R_{b}} \\
\left|\tilde{Q}_{b}-Q(0)\right|<\varepsilon^{*}(\eta), \tilde{Q}_{b}\left(R_{b}\right)=0
\end{array}\right.
$$

with $R_{b}=\frac{2}{|b|} \sqrt{1-\eta}, B_{R_{b}}=\left\{y \in \mathbb{R}^{d},|y| \leq R_{b}\right\}$. Let $\phi_{b}(y)$ be a regular radially symmetric cut-off function satisfying

$$
\phi_{b}(y)=\left\{\begin{array}{lll}
1 & \text { if } \quad|x| \leq R_{b}^{-}=\sqrt{1-\eta} R_{b}, & 0 \leq \phi_{b} \leq 1, \\
0 & \text { if } \quad|x| \geq R_{b},
\end{array}\right.
$$

and $\left\|\nabla \phi_{b}\right\|_{L^{\infty}}+\left\|\Delta \phi_{b}\right\|_{L^{\infty}} \rightarrow 0$ as $|b| \rightarrow 0$. Moreover, let

$$
Q_{b}(r)=\tilde{Q}_{b}(r) \phi_{b}(r),
$$

then

$$
\begin{array}{r}
\left\|e^{C r}\left(Q_{b}-Q\right)\right\|_{H^{10} \cap C^{2}} \rightarrow 0 \quad \text { as } \quad|b| \rightarrow 0, \\
\left\|e^{c r}\left(\frac{\partial Q_{b}}{\partial b}+i \frac{|y|^{2}}{4} Q\right)\right\|_{C^{2}} \rightarrow 0 \quad \text { as } \quad|b| \rightarrow 0, \\
\left|E\left(Q_{b}\right)\right| \leq e^{-\frac{C}{|b|}},
\end{array}
$$

and $Q_{b}$ has super-critical mass:

$$
0<\left.\frac{d}{d\left(b^{2}\right)}\left(\int\left|Q_{b}\right|^{2}\right)\right|_{b^{2}=0}=d_{0}<+\infty .
$$

Remark 1.7. The profiles $Q_{b}$ are not exact self-similar solutions and we define the error term $\Psi_{b}$ by:

$$
\Delta Q_{b}-Q_{b}+i b \Lambda Q_{b}+Q_{b}\left|Q_{b}\right|^{\frac{4}{d}}=-\Psi_{b}
$$

Indeed,

$$
-\Psi_{b}=2 \nabla \tilde{Q}_{b} \cdot \nabla \phi_{b}+\tilde{Q}_{b}\left(\Delta \phi_{b}\right)+i b \tilde{Q}_{b}\left(\Lambda \phi_{b}\right)+\left(\phi_{b}^{1+\frac{4}{d}}-\phi_{b}\right)\left|\tilde{Q}_{b}\right|^{\frac{4}{d}} \tilde{Q}_{b} .
$$

Next, we introduce the outgoing radiation escaping the soliton core according to the following Lemma.

Lemma 1.8 (Linear outgoing radiation, [19](Lemma 15)). There exist universal constants $C>0$ and $\eta^{*}>0$ such that $\forall 0<\eta<\eta^{*}$, there exists $b^{*}>0$ such that for any $|b|<b^{*}$, the following holds true: with $\Psi_{b}$ given by (1.31), there exists a unique radiation solution $\zeta_{b}$ to

$$
\left\{\begin{array}{l}
\Delta \zeta_{b}-\zeta_{b}+i b \Lambda \zeta_{b}=\Psi_{b} \\
\int\left|\nabla \zeta_{b}\right|^{2}<+\infty
\end{array}\right.
$$

Moreover, let

$$
\Gamma_{b}:=\lim _{|y| \rightarrow+\infty}|y|^{d}\left|\zeta_{b}(y)\right|^{2},
$$

then,

$$
e^{-(1+C \eta) \frac{\pi}{|b|}} \leq \Gamma_{b} \leq e^{-(1-C \eta) \frac{\pi}{|b|}} .
$$

More precisely, it follows that

$$
\left\||y|^{\frac{d}{2}}\left(\left|\zeta_{b}\right|+|y|\left|\nabla \zeta_{b}\right|\right)\right\|_{L^{\infty}\left(|y| \geq R_{b}\right)} \leq \Gamma_{b}^{\frac{1}{2}-C \eta}, \quad \int\left|\nabla \zeta_{b}\right|^{2} \leq \Gamma_{b}^{1-C \eta} .
$$


For $|y|$ small, we have: For any $\sigma \in(0,5)$, there exists $\eta^{* *}(\sigma)$, such that for any $0<\eta<\eta^{* *}$, there exists $b^{* *}(\eta)$ such that for any $0<|b|<b^{* *}(\eta)$, it follows that

$$
\left\|\zeta_{b} e^{-\frac{\sigma \theta(b|y|)}{|b|}}\right\|_{\mathcal{C}^{2}\left(|y| \leq R_{b}\right)} \leq \Gamma_{b}^{\frac{1}{2}+\frac{\sigma}{10}} .
$$

Last, $\zeta_{b}$ is differentiable with respect to $b$ with estimate

$$
\left\|\partial_{b} \zeta_{b}\right\|_{\mathcal{C}^{1}} \leq \Gamma_{b}^{\frac{1}{2}-C \eta}
$$

Next, let us introduce some notations in the I-method, which consists in smoothing out the $H^{s}$-initial data with $0<s<1$ in order to access a good local theory available at the $H^{1}$-regularity. To do it, for $N \gg 1$, we define the Fourier multiplier $I_{N}$ by

$$
\widehat{I_{N} u}(\xi):=m_{N}(\xi) \hat{u}(\xi),
$$

where $m_{N}(\xi)$ is a smooth radial decreasing cut off function such that

$$
m_{N}(\xi)= \begin{cases}1, & |\xi| \leq N \\ \left(\frac{|\xi|}{N}\right)^{s-1}, & |\xi| \geq 2 N\end{cases}
$$

Thus, $I_{N}$ is the identity operator on frequencies $|\xi| \leq N$ and behaves like a fractional integral operator of order $1-s$ on higher frequencies. It is easy to show that the operator $I_{N}$ maps $H^{s}$ to $H^{1}$. Moreover, we have

$$
\|u\|_{H^{s}} \lesssim\left\|I_{N} u\right\|_{H^{1}} \lesssim N^{1-s}\|u\|_{H^{s}} .
$$

Let $\delta_{\lambda} f(x)=f(x / \lambda)$. Then, by a simple computation, we have

$$
\left(I_{N} \delta_{\lambda} f\right)(x)=\left(\delta_{\lambda} I_{N \lambda} f\right)(x) .
$$

By a simple argument as in [5] and perturbation theory, we can reduce Theorem 1.4 to the following proposition.

Proposition 1.9 (Explicit description of the blowup set). Let $s>s(d)$ and consider an initial data

$$
u_{0}=G(0)+H(0), G(0) \in H^{1}, H(0) \in H^{s}
$$

such that $G(0)$ admits a geometrical decomposition:

$$
G(0, x)=\frac{1}{\lambda(0)^{\frac{d}{2}}}\left(Q_{b(0)}+g(0)\right)\left(\frac{x-x(0)}{\lambda(0)}\right) e^{-i \gamma(0)},
$$

with the following controls:

(i) Control of the scaling parameter:

$$
0<b(0) \ll 1,0<\lambda(0) \leq e^{-e^{\frac{2 \pi}{3 b(0)}}},
$$

(ii) $L^{2}$-control of the excess of mass:

$$
\|g(0)\|_{L^{2}}+\|H(0)\|_{L^{2}} \ll 1,
$$

(iii) $H^{s}$-control of the rough excess of mass:

$$
\|H(0)\|_{H^{s}} \leq \lambda(0)^{10},
$$

(iv) $H^{1}$-smallness of $g(0)$ :

$$
\int|\nabla g(0)|^{2}+\int|g(0)|^{2} e^{-|y|} \leq \Gamma_{b(0)}^{\frac{3}{4}},
$$


(v) Control of the conservation laws for the $H^{1}$-part:

$$
\begin{aligned}
& |E(G(0))| \leq \frac{1}{\sqrt{\lambda(0)}}, \\
& |P(G(0))| \leq \frac{1}{\sqrt{\lambda(0)}} .
\end{aligned}
$$

Then, the corresponding $H^{s}$ solution $u(t)$ to (1.1) blows up in finite time $0<T<$ $+\infty$ in the log-log regime and the conclusions of Theorem 1.4 hold true.

Hence, our goal is to prove Proposition [1.9, Let $u_{0} \in H^{s}$ satisfy the hypotheses of Proposition 1.9. We can rewrite the decomposition (1.38) as

$$
u(0, x)=\frac{1}{\lambda(0)^{\frac{d}{2}}}\left(Q_{b(0)}+\varepsilon(0)\right)\left(\frac{x-x(0)}{\lambda(0)}\right) e^{-i \gamma(0)}
$$

with $\varepsilon(0)=g(0)+h(0)$ and

$$
H(0, x)=\frac{1}{\lambda(0)^{\frac{d}{2}}} h\left(0, \frac{x-x(0)}{\lambda(0)}\right) e^{-i \gamma(0)} .
$$

We have by (1.42)

$$
\|h(0)\|_{\dot{H}^{s}}=\lambda(0)^{s}\|H(0)\|_{\dot{H}^{s}} \leq \lambda(0)^{10+s} .
$$

This together with (1.41) and (1.43) yields that

$$
\|\varepsilon(0)\|_{H^{s}} \leq\|g(0)\|_{H^{s}}+\|h(0)\|_{H^{s}} \ll 1 .
$$

Next, we derive a frequency localized version of (1.43) for $\varepsilon(0)$. Set

$$
N(0)=\left(\frac{1}{\lambda(0)}\right)^{\frac{1}{\beta}}
$$

with $\beta$ given in Remark 1.10, then,

$$
1 \ll\left(\frac{1}{\lambda(0)}\right)^{\frac{1-\beta}{\beta}}=N(0) \lambda(0) .
$$

And so, using (1.34), (1.36), (1.40) and (1.48), we get

$$
\int\left|I_{N(0) \lambda(0)} \nabla h(0)\right|^{2} \lesssim(N(0) \lambda(0))^{2(1-s)}\|h(0)\|_{\dot{H}^{s}}^{2} \lesssim \lambda(0)^{10} \leq \Gamma_{b(0)}^{10} .
$$

This together with (1.42) and (1.43) implies

$$
\int\left|I_{N(0) \lambda(0)} \nabla \varepsilon(0)\right|^{2}+\int|\varepsilon(0)|^{2} e^{-|y|} \leq \Gamma_{b(0)}^{\frac{3}{4}} .
$$

Remark 1.10. The restriction on $\beta$ stems from two sides. One comes from Corollary 2.10 below:

$$
\frac{4 s}{\min \{4, d\} s-4(1-s)}<\frac{1}{\beta} .
$$

Another comes from Lemma 3.11 below

$$
\frac{1}{\beta}>\frac{4 s}{\min \{4, d\} s^{2}-(1-s)}
$$

to guarantee the convergence of the summation. Hence, we will take $\beta \in(0,1)$ such that

$$
\frac{1}{\beta}>\min \left\{\frac{4 s}{\min \{4, d\} s-4(1-s)} \frac{4 s}{\min \{4, d\} s^{2}-(1-s)}\right\} .
$$


Lemma 1.11 (Nonlinear modulation theory, [17, 18, 20]). There exists $\alpha_{2}>0$ such that for $\alpha_{0}<\alpha_{2}$, there exist some functions $(\lambda, \gamma, x, b)$ : $[0, T) \rightarrow(0,+\infty) \times$ $\mathbb{R} \times \mathbb{R}^{d} \times \mathbb{R}$ such that

$$
\varepsilon(t, y)=e^{i \gamma(t)} \lambda(t)^{\frac{d}{2}} u(t, \lambda(t) y+x(t))-Q_{b(t)}(y)
$$

satisfies the following orthogonality conditions:

$$
\begin{aligned}
\left(\varepsilon_{1}(t),|y|^{2} \Sigma\right)+\left(\varepsilon_{2}(t),|y|^{2} \Theta\right) & =0, \\
\left(\varepsilon_{1}(t), y \Sigma\right)+\left(\varepsilon_{2}(t), y \Theta\right) & =0, \\
-\left(\varepsilon_{1}(t), \Lambda \Theta\right)+\left(\varepsilon_{2}(t), \Lambda \Sigma\right) & =0, \\
-\left(\varepsilon_{1}(t), \Lambda^{2} \Theta\right)+\left(\varepsilon_{2}(t), \Lambda^{2} \Sigma\right) & =0,
\end{aligned}
$$

where $\varepsilon=\varepsilon_{1}+i \varepsilon_{2}$ and $Q_{b(t)}=\Sigma+i \Theta$ in terms of real and imaginary part.

Remark 1.12. The existence of such a decomposition (1.53) requires only the smallness of the local $L^{2}$-norm of $\varepsilon$ due to the regularity of $Q_{b}$ and its fast decay in space. We note that (1.52) ensures that the deformed parameters ensuring the orthogonality conditions at time $t=0$ are exponentially small in $b(0)$ compared to (1.46). We shall thus abuse notations at time $t=0$ and identify these two decompositions which satisfy the initialized control of Proposition 1.9.

Our main claim now is that the controls of Proposition 1.9 determine a trapped dynamical region. In other words, we claim the following bootstrapped estimates. Consider a time interval $\left[0, T^{+}\right]$such that the solution $u(t)$ admits a decomposition

$$
u(t, x)=\frac{1}{\lambda(t)^{\frac{d}{2}}}\left(Q_{b(t)}(\cdot)+\varepsilon(t, \cdot)\right)\left(\frac{x-x(t)}{\lambda(t)}\right) e^{-i \gamma(t)}, t \in\left[0, T^{+}\right]
$$

satisfying the orthogonality conditions (1.54)-(1.57). Now, let us assume the following uniform controls on $\left[0, T^{+}\right]$:

(i) Control of $b(t)$ and the $L^{2}$ mass:

$$
b(t)>0 \quad \text { and } \quad b(t)+\|\varepsilon(t)\|_{L^{2}} \leq 10\left(b(0)+\|\varepsilon(0)\|_{L^{2}}\right) ;
$$

(ii) Control and monotonicity of the scaling parameter:

$$
\lambda(t) \leq e^{-e^{\frac{\pi}{100 b(t)}}}
$$

and almost monotonicity:

$$
\forall 0<t_{1} \leq t_{2} \leq T^{+}, \lambda\left(t_{2}\right) \leq \frac{3}{2} \lambda\left(t_{1}\right) .
$$

Let $k_{0} \leq k^{+}$integers such that

$$
\frac{1}{2^{k_{0}}} \leq \lambda(0) \leq \frac{1}{2^{k_{0}-1}}, \frac{1}{2^{k^{+}}} \leq \lambda\left(T^{+}\right) \leq \frac{1}{2^{k^{+}-1}},
$$

and for $k_{0} \leq k \leq k^{+}$, let $t_{k}$ be a time such that

$$
\lambda\left(t_{k}\right)=\frac{1}{2^{k}},
$$

then, we assume the control of the doubling time interval:

$$
t_{k+1}-t_{k} \leq k \lambda\left(t_{k}\right)^{2} .
$$


(iii) Frequency localized control of the excess of mass: let

$$
N(t)=\left(\frac{1}{\lambda(t)}\right)^{\frac{1}{\beta}}
$$

then,

$$
\int\left|I_{N(t) \lambda(t)} \nabla \varepsilon(t)\right|^{2}+\int|\varepsilon(t)|^{2} e^{-|y|} \leq \Gamma_{b(t)}^{\frac{1}{4}} .
$$

We then claim the following Lemma which is the main step of the proof of Proposition 1.9 and states that all above estimates may be improved:

Lemma 1.13 (Bootstrap lemma). There holds the following uniform control on $\left[0, T^{+}\right]$:

$$
\begin{aligned}
b(t)>0 \quad \text { and } \quad b(t)+\|\varepsilon(t)\|_{L^{2}} & \leq 5\left(b(0)+\|\varepsilon(0)\|_{L^{2}}\right), \\
\lambda(t) & \leq e^{-e^{\frac{\pi}{10 b(t)}},} \\
\forall 0<t_{1} \leq t_{2} \leq T^{+}, \lambda\left(t_{2}\right) & \leq \frac{5}{4} \lambda\left(t_{1}\right), \\
t_{k+1}-t_{k} & \leq \sqrt{k} \lambda\left(t_{k}\right)^{2}, \\
\int\left|I_{N(t) \lambda(t)} \nabla \varepsilon(t)\right|^{2}+\int|\varepsilon(t)|^{2} e^{-|y|} & \leq \Gamma_{b(t)}^{\frac{2}{3}} .
\end{aligned}
$$

Remark 1.14. By (1.36), and the bootstrapped estimates (1.59), (1.65), (1.66), we obtain

$$
\|\varepsilon(t)\|_{H^{s}} \lesssim\left\|I_{N(t) \lambda(t)} \varepsilon(t)\right\|_{H^{1}} \ll 1 .
$$

This together with the geometrical decomposition (1.58), (1.27) yields that

$$
\|u(t)\|_{H^{s}}=\frac{\left\|Q_{b(t)}+\varepsilon(t)\right\|_{H^{s}}}{\lambda(t)^{s}} \sim \frac{1}{\lambda(t)^{s}} .
$$

\section{Notation and Almost conservation law}

2.1. Some notation. For nonnegative quantities $X$ and $Y$, we will write $X \lesssim Y$ to denote the estimate $X \leq C Y$ for some $C>0$. If $X \lesssim Y \lesssim X$, we will write $X \sim Y$. Dependence of implicit constants on the power $p$ or the dimension will be suppressed; dependence on additional parameters will be indicated by subscripts. For example, $X \lesssim_{u} Y$ indicates $X \leq C Y$ for some $C=C(u)$. We denote $a_{ \pm}$as $a \pm \epsilon$ with $0<\epsilon \ll 1$.

For a spacetime slab $I \times \mathbb{R}^{d}$, we write $L_{t}^{q} L_{x}^{r}\left(I \times \mathbb{R}^{d}\right)$ for the Banach space of functions $u: I \times \mathbb{R}^{d} \rightarrow \mathbb{C}$ equipped with the norm

$$
\|u\|_{L_{t}^{q} L_{x}^{r}\left(I \times \mathbb{R}^{d}\right)}:=\left(\int_{I}\|u(t)\|_{L_{x}^{r}\left(\mathbb{R}^{d}\right)}\right)^{1 / q},
$$

with the usual adjustments when $q$ or $r$ is infinity. When $q=r$, we abbreviate $L_{t}^{q} L_{x}^{q}=L_{t, x}^{q}$. We will also often abbreviate $\|f\|_{L_{x}^{r}\left(\mathbb{R}^{d}\right)}$ to $\|f\|_{L_{x}^{r}}$. For $1 \leq r \leq \infty$, we use $r^{\prime}$ to denote the dual exponent to $r$, i.e. the solution to $\frac{1}{r}+\frac{1}{r^{\prime}}=1$.

The Fourier transform on $\mathbb{R}^{d}$ is defined by

$$
\widehat{f}(\xi):=(2 \pi)^{-\frac{d}{2}} \int_{\mathbb{R}^{d}} e^{-i x \cdot \xi} f(x) d x,
$$


giving rise to the fractional differentiation operators $|\nabla|^{s}$ and $\langle\nabla\rangle^{s}$, defined by

$$
\widehat{|\nabla|^{s} f}(\xi):=|\xi|^{s} \hat{f}(\xi), \widehat{\langle\nabla\rangle^{s} f}(\xi):=\langle\xi\rangle^{s} \hat{f}(\xi),
$$

where $\langle\xi\rangle:=1+|\xi|$. This helps us to define the homogeneous and inhomogeneous Sobolev norms

$$
\|f\|_{\dot{H}_{x}^{s}\left(\mathbb{R}^{d}\right)}:=\left\||\xi|^{s} \hat{f}\right\|_{L_{x}^{2}\left(\mathbb{R}^{d}\right)},\|f\|_{H_{x}^{s}\left(\mathbb{R}^{d}\right)}:=\left\|\langle\xi\rangle^{s} \hat{f}\right\|_{L_{x}^{2}\left(\mathbb{R}^{d}\right)} .
$$

We will also need the Littlewood-Paley projection operators. Specifically, let $\varphi(\xi)$ be a smooth bump function adapted to the ball $|\xi| \leq 2$ which equals 1 on the ball $|\xi| \leq 1$. For each dyadic number $N \in 2^{\mathbb{Z}}$, we define the Littlewood-Paley operators

$$
\begin{aligned}
\widehat{P_{\leq N} f}(\xi) & :=\varphi\left(\frac{\xi}{N}\right) \widehat{f}(\xi), \\
\widehat{P_{>N} f}(\xi) & :=\left(1-\varphi\left(\frac{\xi}{N}\right)\right) \widehat{f}(\xi), \\
\widehat{P_{N} f}(\xi) & :=\left(\varphi\left(\frac{\xi}{N}\right)-\varphi\left(\frac{2 \xi}{N}\right)\right) \widehat{f}(\xi) .
\end{aligned}
$$

Similarly we can define $P_{<N}, P_{\geq N}$, and $P_{M<\cdot \leq N}=P_{\leq N}-P_{\leq M}$, whenever $M$ and $N$ are dyadic numbers. We will frequently write $f_{\leq N}$ for $P_{\leq N} f$ and similarly for the other operators.

The Littlewood-Paley operators commute with derivative operators, the free propagator, and the conjugation operation. They are self-adjoint and bounded on every $L_{x}^{p}$ and $\dot{H}_{x}^{s}$ space for $1 \leq p \leq \infty$ and $s \geq 0$, moreover, they also obey the following Bernstein estimates

$$
\begin{aligned}
& \left\|P_{\geq N} f\right\|_{L^{p}} \lesssim N^{-s}\left\||\nabla|^{s} P_{\geq N} f\right\|_{L^{p}}, \\
& \left\||\nabla|^{s} P_{\leq N} f\right\|_{L^{p}} \lesssim N^{s}\left\|P_{\leq N} f\right\|_{L^{p}}, \\
& \left\||\nabla|^{ \pm s} P_{N} f\right\|_{L^{p}} \sim N^{ \pm s}\left\|P_{N} f\right\|_{L^{p}}, \\
& \left\|P_{\leq N} f\right\|_{L^{q}} \lesssim N^{\frac{d}{p}-\frac{d}{q}}\left\|P_{\leq N} f\right\|_{L^{p}}, \\
& \left\|P_{N} f\right\|_{L^{q}} \lesssim N^{\frac{d}{p}-\frac{d}{q}}\left\|P_{N} f\right\|_{L^{p}},
\end{aligned}
$$

where $s \geq 0$ and $1 \leq p \leq q \leq \infty$.

We will also use the following basic inequalities.

Lemma 2.1 ([17]). For any $z \in \mathbb{C}$ with $z=z_{1}+i z_{2}$, there holds

$$
\begin{aligned}
& \left|\left(1+z_{1}\right)\right| 1+\left.z\right|^{\frac{4}{d}}-1-\left(\frac{4}{d}+1\right) z_{1}+i z_{2}\left(|1+z|^{\frac{4}{d}}-1\right) \mid \\
\leq & \left\{\begin{array}{l}
C\left(|z|^{1+\frac{4}{3}}+|z|^{2}\right) \quad \text { if } \quad d=3 \\
C|z|^{2} \quad \text { if } \quad d \geq 4,
\end{array}\right.
\end{aligned}
$$

and

$$
\begin{aligned}
& \left|\left(1+z_{1}\right)\right| 1+\left.z\right|^{\frac{4}{d}}-1-\left(\frac{4}{d}+1\right) z_{1}-\frac{2}{d}\left(\frac{4}{d}+1\right) z_{1}^{2}-\frac{2}{d} z_{2}^{2} \mid \\
\leq & \left\{\begin{array}{l}
C|z|^{3} \quad \text { if } \quad d=3 \\
C|z|^{2+\frac{2}{d}} \quad \text { if } \quad d \geq 4,
\end{array}\right.
\end{aligned}
$$


and

$$
\begin{aligned}
& || 1+\left.z\right|^{2+\frac{4}{d}}-1-\left(\frac{4}{d}+2\right) z_{1}-\left(\frac{2}{d}+1\right)\left(\frac{4}{d}+1\right) z_{1}^{2}-\left(\frac{2}{d}+1\right) z_{2}^{2} \mid \\
\leq & \left\{\begin{array}{l}
C\left(|z|^{2+\frac{4}{3}}+|z|^{3}\right) \quad \text { if } \quad d=3 \\
C|z|^{3} \quad \text { if } \quad d \geq 4 .
\end{array}\right.
\end{aligned}
$$

2.2. Nonlinear estimate. For $N>1$, we define the Fourier multiplier $I_{N}$ given by

$$
\widehat{I_{N} u}(\xi):=m_{N}(\xi) \hat{u}(\xi),
$$

where $m_{N}(\xi)$ is a smooth radial decreasing cut off function by (1.35). Let us collect basic properties of $I_{N}$.

Lemma 2.2 ([27]). Let $1<p<\infty$ and $0 \leq \sigma \leq s<1$. Then,

$$
\begin{aligned}
\left\|I_{N} f\right\|_{L^{p}} & \lesssim\|f\|_{L^{p}}, \\
\left\||\nabla|^{\sigma} P_{>N} f\right\|_{L^{p}} & \lesssim N^{\sigma-1}\left\|\nabla I_{N} f\right\|_{L^{p}}, \\
\|f\|_{H^{s}} \lesssim\left\|I_{N} f\right\|_{H^{1}} & \lesssim N^{1-s}\|f\|_{H^{s}} .
\end{aligned}
$$

We will need the following fractional calculus estimates from [3].

Lemma 2.3 (Fractional product rule [3]). Let $s \geq 0$, and $1<r, r_{j}, q_{j}<\infty$ satisfy $\frac{1}{r}=\frac{1}{r_{i}}+\frac{1}{q_{i}}$ for $i=1,2$. Then

$$
\left\||\nabla|^{s}(f g)\right\|_{L_{x}^{r}\left(\mathbb{R}^{d}\right)} \lesssim\|f\|_{L_{x}^{r_{1}}\left(\mathbb{R}^{d}\right)}\left\||\nabla|^{s} g\right\|_{L_{x}^{q_{1}}\left(\mathbb{R}^{d}\right)}+\left\||\nabla|^{s} f\right\|_{L_{x}^{r_{2}}\left(\mathbb{R}^{d}\right)}\|g\|_{L_{x}^{q_{2}}\left(\mathbb{R}^{d}\right)} .
$$

From [5], we have

$$
\left\|I_{N} \nabla(f g)\right\|_{L_{x}^{r}\left(\mathbb{R}^{d}\right)} \lesssim\|f\|_{L_{x}^{r_{1}}\left(\mathbb{R}^{d}\right)}\left\|I_{N} \nabla g\right\|_{L_{x}^{q_{1}\left(\mathbb{R}^{d}\right)}}+\left\|I_{N} \nabla f\right\|_{L_{x}^{r_{2}\left(\mathbb{R}^{d}\right)}}\|g\|_{L_{x}^{q_{2}\left(\mathbb{R}^{d}\right)}} .
$$

Lemma 2.4 (Fractional chain rule [3]). Let $G \in C^{1}(\mathbb{C}), s \in(0,1]$, and $1<$ $r, r_{1}, r_{2}<+\infty$ satisfy $\frac{1}{r}=\frac{1}{r_{1}}+\frac{1}{r_{2}}$. Then

$$
\left\||\nabla|^{s} G(u)\right\|_{r} \lesssim\left\|G^{\prime}(u)\right\|_{r_{1}}\left\||\nabla|^{s} u\right\|_{r_{2}} .
$$

As noted in the introduction, one needs to estimate the commutator $|I u|^{p} I u-$ $I\left(|u|^{p} u\right)$ in the increment of modified energy $E(I u)(t)$. When $p$ is an even integer, one can use multilinear analysis to expand this commutator into a product of Fourier transforms of $u$ and $I u$, and carefully measure frequency interactions to derive an estimate (see for example 44). However, this is not possible when $p$ in not an even integer. Instead, Visan and Zhang in 27] established the following rougher (weaker, but more robust) estimate:

Lemma 2.5 (commutator estimate, [27). Let $1<r, r_{1}, r_{2}<\infty$ be such that $\frac{1}{r}=\frac{1}{r_{1}}+\frac{1}{r_{2}}$ and let $0<\nu<s$. Then,

$$
\left\|I_{N}(f g)-\left(I_{N} f\right) g\right\|_{L^{r}} \lesssim N^{-(1-s+\nu)}\left\|I_{N} f\right\|_{L^{r_{1}}}\left\|\langle\nabla\rangle^{1-s+\nu} g\right\|_{L^{r_{2}}} .
$$

Furthermore, let I be a time interval and let $\frac{1}{1+\min \left\{1, \frac{4}{d}\right\}}<s<1$, then we have

$$
\begin{array}{r}
\left\|\nabla I_{N}\left(|u|^{\frac{4}{d}} u\right)-\left(I_{N} \nabla u\right)|u|^{\frac{4}{d}}\right\|_{L_{t}^{2} L_{x}^{\frac{2 d}{d+2}}\left(I \times \mathbb{R}^{d}\right)} \lesssim N^{-\min \left\{1, \frac{4}{d}\right\} s_{+}}\left\|\langle\nabla\rangle I_{N} u\right\|_{S^{0}(I)}^{1+\frac{4}{d}}, \\
\left\|\langle\nabla\rangle I_{N}\left(|u|^{\frac{4}{d}} u\right)\right\|_{N^{0}(I)} \lesssim\left(|I|^{\frac{2 s}{d}}+N^{-\min \left\{1, \frac{4}{d}\right\} s_{+}}\right)\left\|\langle\nabla\rangle I_{N} u\right\|_{S^{0}(I)}^{1+\frac{4}{d}}, \\
\left\|\langle\nabla\rangle^{\min \left\{1, \frac{4}{d}\right\} s_{-}}\left(|u|^{\frac{4}{d}}\right)\right\|_{L_{t}^{\infty} L_{x}^{\frac{d}{2}}} \lesssim\left\|\langle\nabla\rangle I_{N} u\right\|_{L_{t}^{\infty} L_{x}^{2}},
\end{array}
$$


where $S^{0}(I)$ and $N^{0}(I)$ is defined in Definition 2.7 below.

Remark 2.6. It is easy to check that for $(q, r) \in \Lambda_{0}$,

$$
\left\|\langle\nabla\rangle^{s} u\right\|_{L_{t}^{q} L_{x}^{r}} \lesssim\left\|\langle\nabla\rangle I_{N} u\right\|_{L_{t}^{q} L_{x}^{r}},
$$

where $\Lambda_{0}$ is defined in Definition 2.7 below.

2.3. Strichartz estimates and local well-posedness. In this subsection, we consider the Cauchy problem

$$
\left\{\begin{array}{l}
i u_{t}+\Delta u-f(u)=0, \\
u(0)=u_{0} .
\end{array}\right.
$$

The integral equation for the Cauchy problem (2.15) can be written as

$$
u(t, x)=e^{i\left(t-t_{0}\right) \Delta} u\left(t_{0}\right)-i \int_{t_{0}}^{t} e^{i(t-s) \Delta} f(u(s)) d s .
$$

Now we recall the dispersive estimate for the free Schrödinger operator $U(t)=$ $e^{i t \Delta}$. From the explicit formula

$$
e^{i t \Delta} f(x)=\frac{1}{(4 i \pi t)^{\frac{d}{2}}} \int_{\mathbb{R}^{d}} e^{i \frac{|x-y|^{2}}{4 t}} f(y) d y,
$$

it is easy to get the standard dispersive inequality

$$
\left\|e^{i t \Delta} f\right\|_{L_{x}^{\infty}\left(\mathbb{R}^{d}\right)} \lesssim|t|^{-\frac{d}{2}}\|f\|_{L_{x}^{1}\left(\mathbb{R}^{d}\right)}
$$

for all $t \neq 0$. On the other hand, since the free operator conserves the $L_{x}^{2}\left(\mathbb{R}^{d}\right)$-norm, we obtain by interpolation

$$
\left\|e^{i t \Delta} f\right\|_{L_{x}^{q}\left(\mathbb{R}^{d}\right)} \leq C|t|^{-d\left(\frac{1}{2}-\frac{1}{q}\right)}\|f\|_{L_{x}^{q^{\prime}\left(\mathbb{R}^{d}\right)}}
$$

for all $t \neq 0$ and $2 \leq q \leq+\infty, \frac{1}{q}+\frac{1}{q^{\prime}}=1$.

The Strichartz estimates involve the following definitions:

Definition 2.7. A pair of Lebesgue space exponents $(q, r)$ are called Schrödinger admissible for $\mathbb{R}^{d+1}$, or denote by $(q, r) \in \Lambda_{0}$ when $q, r \geq 2,(q, r, d) \neq(2, \infty, 2)$, and

$$
\frac{2}{q}=d\left(\frac{1}{2}-\frac{1}{r}\right)
$$

For a fixed spacetime slab $I \times \mathbb{R}^{d}$, we define the Strichartz norm

$$
\|u\|_{S^{0}(I)}:=\sup _{(q, r) \in \Lambda_{0}}\|u\|_{L_{t}^{q} L_{x}^{r}\left(I \times \mathbb{R}^{d}\right)}, d \geq 3
$$

We denote $S^{0}(I)$ to be the closure of all test functions under this norm and write $N^{0}(I)$ for the dual of $S^{0}(I)$.

According to the above dispersive estimate, the abstract duality and interpolation argument(see [11]), we have the following Strichartz estimates.

Lemma 2.8 (Strichartz estimate, [10, 11]). Let $s \geq 0$, and let I be a compact time interval, and let $u: I \times \mathbb{R}^{d} \rightarrow \mathbb{C}$ be a solution of the Schrödinger equation

$$
i u_{t}+\Delta u+h=0 .
$$

Then, for all $t_{0} \in I$

$$
\left\||\nabla|^{s} u\right\|_{S^{0}(I)} \leq C\left\||\nabla|^{s} u\left(t_{0}\right)\right\|_{L_{x}^{2}\left(\mathbb{R}^{d}\right)}+\left\||\nabla|^{s} h\right\|_{N^{0}(I)} .
$$


By the fixed point argument, we have the following local well-posedness(LWP).

Lemma $2.9\left(H^{s}\right.$-LWP $)$. Let $s \in(0,1], u_{0} \in H^{s}\left(\mathbb{R}^{d}\right)$ and

$$
T_{\mathrm{LWP}}=c\left\|u_{0}\right\|_{H^{s}}^{-\frac{2}{s}}
$$

with $c$ small depending the constant in Strichartz estimate and Sobolev embedding. Then, there exists a unique solution $u(t)$ to (1.1) on [0, $\left.T_{\mathrm{LWP}}\right]$ and satisfying

$$
\|u\|_{S^{0}\left(\left[0, T_{\mathrm{LWP}}\right]\right)} \leq 2 C\left\|u_{0}\right\|_{L_{x}^{2}},\left.\left\|\langle\nabla\rangle^{s} u\right\|\right|_{S^{0}\left(\left[0, T_{\mathrm{LWP}}\right]\right)} \leq 2 C\left\|u_{0}\right\|_{H^{s}} .
$$

Proof. We apply the Banach fixed point argument to prove this lemma. First, we define the map

$$
\Phi(u)=e^{i t \Delta} u_{0}-i \int_{0}^{t} e^{i(t-s) \Delta}\left(|u|^{\frac{4}{d}} u\right)(s) d s
$$

on the complete metric space $B$

$B:=\left\{u \in C\left(I ; H^{s}\right):\|u\|_{S^{0}\left(\left[0, T_{\mathrm{LWP}}\right]\right)} \leq 2 C\left\|u_{0}\right\|_{L_{x}^{2}},\left\|\langle\nabla\rangle^{s} u\right\|\left\|_{S^{0}\left(\left[0, T_{\mathrm{LWP}}\right]\right)} \leq 2 C\right\| u_{0} \|_{H^{s}}\right\}$

with the metric $d(u, v)=\|u-v\|_{L_{t, x} \frac{2(d+2)}{d}\left(\left[0, T_{\mathrm{LWP}}\right] \times \mathbb{R}^{d}\right)}$, where $C$ is the constant in Strichartz estimates.

It suffices to prove that the operator $\Phi(u)$ is a contraction map on $B$ for $\left[0, T_{\mathrm{LWP}}\right]$. In fact, if $u \in B$, then by Strichartz estimate, Hölder's inequality and Sobolev embedding, we have

$$
\begin{aligned}
& \|\Phi(u)\|_{S^{0}\left(\left[0, T_{\mathrm{LWP}}\right]\right)} \leq C\left\|u_{0}\right\|_{L_{x}^{2}}+C\left\||u|^{\frac{4}{d}} u\right\|_{L_{t, x}^{\frac{2(d+2)}{d+4}}\left(\left[0, T_{\mathrm{LWP}}\right] \times \mathbb{R}^{d}\right)} \\
& \leq C\left\|u_{0}\right\|_{L_{x}^{2}}+C\|u\|_{L_{t, x}^{\frac{2(d+2)}{d}}\left(\left[0, T_{\mathrm{LWP}}\right] \times \mathbb{R}^{d}\right)}\|u\|_{L_{t, x}^{\frac{4}{d}}}^{\frac{2(d+2)}{d}}\left(\left[0, T_{\mathrm{LWP}}\right] \times \mathbb{R}^{d}\right) \\
& \leq C\left\|u_{0}\right\|_{L_{x}^{2}}+C\|u\|_{L_{t, x}^{\frac{2(d+2)}{d}}\left(\left[0, T_{\mathrm{LWP}}\right] \times \mathbb{R}^{d}\right)}\left(\left.T_{\mathrm{LWP}}^{\frac{s}{2}}\left\|\langle\nabla\rangle^{s} u\right\|\right|_{S^{0}\left(\left[0, T_{\mathrm{LWP}}\right]\right)}\right)^{\frac{4}{d}} \\
& \leq C\left\|u_{0}\right\|_{L_{x}^{2}}+2^{\frac{4}{d}} C^{2+\frac{4}{d}} C^{\frac{4}{d}}\left\|u_{0}\right\|_{L_{x}^{2}} \\
& \leq 2 C\left\|u_{0}\right\|_{L_{x}^{2}}
\end{aligned}
$$

provided that $2^{\frac{4}{d}} C^{2+\frac{4}{d}} c^{\frac{4}{d}}<1$ with $T_{\mathrm{LWP}}=c\left\|u_{0}\right\|_{H^{s}}^{-\frac{2}{s}}$. Similarly, we obtain

$$
\begin{aligned}
\left.\left\|\langle\nabla\rangle^{s} \Phi(u)\right\|\right|_{S^{0}\left(\left[0, T_{\mathrm{LWP}}\right]\right)} & \leq C\left\|u_{0}\right\|_{H^{s}}+C\left\|\langle\nabla\rangle^{s}\left(|u|^{\frac{4}{d}} u\right)\right\|_{L_{t, x}^{\frac{2(d+2)}{d+4}}\left(\left[0, T_{\mathrm{LWP}}\right] \times \mathbb{R}^{d}\right)} \\
& \leq C\left\|u_{0}\right\|_{H^{s}}+C\left\|\langle\nabla\rangle^{s} u\right\|_{L_{t, x}^{\frac{2(d+2)}{d}}}\left({ }_{\left(\left[0, T_{\mathrm{LWP}}\right] \times \mathbb{R}^{d}\right)}\left(\left.T_{\mathrm{LWP}}^{\frac{s}{2}}\left\|\langle\nabla\rangle^{s} u\right\|\right|_{S^{0}\left(\left[0, T_{\mathrm{LWP}}\right]\right)}\right)^{\frac{4}{d}}\right. \\
& \leq C\left\|u_{0}\right\|_{H^{s}}+2^{\frac{4}{d}} C^{2+\frac{4}{d}} c^{\frac{4}{d}}\left\|u_{0}\right\|_{H^{s}} \leq 2 C\left\|u_{0}\right\|_{L_{x}^{2} .}
\end{aligned}
$$

Hence, $\Phi(u) \in B$. 
On the other hand, for $u, v \in B$, by Strichartz estimate, we obtain

$$
\begin{aligned}
d(\Phi(u), \Phi(v)) & =\|\Phi(u)-\Phi(v)\|_{L_{t, x}^{\frac{2(d+2)}{d}}\left(\left[0, T_{\mathrm{LWP}}\right] \times \mathbb{R}^{d}\right)} \\
& \leq C\left\||u|^{\frac{4}{d}} u-|v|^{\frac{4}{d}} v\right\|_{L_{t, x}^{\frac{2(d+2)}{d+4}}\left(\left[0, T_{\mathrm{LWP}}\right] \times \mathbb{R}^{d}\right)}\|\|_{L_{t, x}^{\frac{2(d+2)}{d}}}\left(\left[0, T_{\left.\mathrm{LWP}] \times \mathbb{R}^{d}\right)}\|(u, v)\|_{L_{t, x}^{\frac{4}{d}}}^{\frac{2(d+2)}{d\left(\left[0, T_{\mathrm{LWP}}\right] \times \mathbb{R}^{d}\right)}}\right.\right. \\
& \leq C\|u-v\|_{L_{t, x}^{\frac{2(d+2)}{d}}\left(\left[0, T_{\mathrm{LWP}}\right] \times \mathbb{R}^{d}\right)}\left(\left.2 T_{\mathrm{LWP}}^{\frac{s}{2}}\left\|\langle\nabla\rangle^{s} u\right\|\right|_{\left.S^{0}\left(\left[0, T_{\mathrm{LWP}}\right]\right)\right)^{\frac{4}{d}}}\right. \\
& \leq C \| u-2^{\frac{8}{d}} C^{2+\frac{4}{d}} c^{\frac{4}{d}} d(u, v) \\
& \leq \frac{1}{2} d(u, v),
\end{aligned}
$$

provided that $2^{\frac{8}{d}} C^{2+\frac{4}{d}} c^{\frac{4}{d}}<\frac{1}{2}$.

Therefore, applying the fixed point theorem gives a unique solution $u$ of (1.1) on $\left[0, T_{\mathrm{LWP}}\right]$ which satisfies the bound (2.21).

Therefore, applying the fixed point theorem gives a unique solution $u$ of (1.1) on $\left[0, T_{\mathrm{LWP}}\right]$ which satisfies the bound (2.21).

Corollary 2.10 ('Modified' $H^{s}$-LWP). For $T^{*} \geq T_{\mathrm{LWP}}$, we denote

$$
\tilde{T}_{\mathrm{LWP}}:=c_{0}\left\|\langle\nabla\rangle I_{N\left(T^{*}\right)} u_{0}\right\|_{L^{2}}^{-\frac{2}{s}}
$$

with $c_{0}$ small. By (2.6), we obtain

$$
\tilde{T}_{\text {LWP }} \leq c_{0}\left\|u_{0}\right\|_{H^{s}}^{-\frac{2}{s}} .
$$

This together with Lemma 2.9 implies that (1.1) is well-posedness on [0, $\left.\tilde{T}_{\mathrm{LWP}}\right]$, and

$$
\|u\|_{S^{0}\left(\left[0, \tilde{T}_{\mathrm{LWP}}\right]\right)} \leq 2 C\left\|u_{0}\right\|_{L_{x}^{2}},\left.\left\|\langle\nabla\rangle^{s} u\right\|\right|_{S^{0}\left(\left[0, \tilde{T}_{\mathrm{LWP}}\right]\right)} \leq 2 C\left\|u_{0}\right\|_{H^{s}} .
$$

Moreover, if $\frac{1}{1+\min \left\{1, \frac{4}{d}\right\}}<s<1, \frac{4 s}{\min \{4, d\} s-4(1-s)}<\frac{1}{\beta}$, then there holds

$$
\left\|\langle\nabla\rangle I_{N} u\right\|_{S^{0}\left(\left[0, \tilde{T}_{\mathrm{LWP}}\right]\right)} \lesssim\left\|I_{N} u\right\|_{H^{1}}
$$

Proof. First, we have by Strichartz estimate and (2.12)

$$
\begin{aligned}
\left\|\langle\nabla\rangle I_{N} u\right\|_{S^{0}\left(\left[0, \tilde{T}_{\mathrm{LWP}}\right]\right) \leq} & C\left\|I_{N} u_{0}\right\|_{H^{1}}+C\left\|\langle\nabla\rangle I_{N}\left(|u|^{\frac{4}{d}} u\right)\right\|_{N^{0}\left(\left[0, \tilde{T}_{\mathrm{LWP}}\right]\right)} \\
\leq & C\left\|I_{N} u_{0}\right\|_{H^{1}}+C \tilde{T}_{\mathrm{LWP}}^{\frac{2 s}{d}}\left\|\langle\nabla\rangle I_{N} u\right\|_{S^{0}\left(\left[0, \tilde{T}_{\mathrm{LWP}}\right]\right)}^{1+\frac{4}{d}} \\
& +C N\left(T^{*}\right)^{-\min \left\{1, \frac{4}{d}\right\} s+\varepsilon}\left\|\langle\nabla\rangle I_{N} u\right\|_{S^{0}\left(\left[0, \frac{\left.\tilde{T}_{\mathrm{LWP}}\right]}{d}\right]\right.}^{1+\frac{4}{2}}
\end{aligned}
$$

for any $\varepsilon>0$ sufficiently small. Using (1.65), monotonicity (1.61), Remark 1.14 (1.73) and (2.6), we get

$$
\begin{aligned}
N\left(T^{*}\right)^{-1} & =\lambda\left(T^{*}\right)^{\frac{1}{\beta}} \lesssim \lambda(0)^{\frac{1}{\beta}} \lesssim\left\|u_{0}\right\|_{H^{s}}^{-\frac{1}{s \beta}} \\
& \lesssim\left(N\left(T^{*}\right)^{s-1}\left\|I_{N\left(T^{*}\right)} u_{0}\right\|_{H^{1}}\right)^{-\frac{1}{s \beta}}
\end{aligned}
$$

and so

$$
N\left(T^{*}\right)^{-1} \lesssim\left\|I_{N\left(T^{*}\right)} u_{0}\right\|_{H^{1}}^{-\frac{1}{s \beta+1-s}} .
$$


Hence, from $\frac{4 s}{\min \{4, d\} s-4(1-s)}<\frac{1}{\beta}$, we know $\frac{4(s \beta+1-s)}{d}-\min \left\{1, \frac{4}{d}\right\} s<0$ and

$$
\begin{aligned}
N\left(T^{*}\right)^{-\min \left\{1, \frac{4}{d}\right\} s+\varepsilon} & =N\left(T^{*}\right)^{-\min \left\{1, \frac{4}{d}\right\} s+\varepsilon+\frac{4(s \beta+1-s)}{d}} N\left(T^{*}\right)^{-\frac{4(s \beta+1-s)}{d}} \\
& \lesssim\left\|I_{N\left(T^{*}\right)} u_{0}\right\|_{H^{1}}^{-\frac{4}{d}} \sim \tilde{T}_{\mathrm{LWP}}^{\frac{2 s}{d}}
\end{aligned}
$$

Plugging this into (2.25) implies

$$
\left\|\langle\nabla\rangle I_{N} u\right\|_{S^{0}\left(\left[0, \tilde{T}_{\mathrm{LWP}]}\right)\right.} \leq C\left\|I_{N} u_{0}\right\|_{H^{1}}+C \tilde{T}_{\mathrm{LWP}}^{\frac{2 s}{d}}\left\|\langle\nabla\rangle I_{N} u\right\|_{S^{0}\left(\left[0, \tilde{T}_{\mathrm{LWP}}\right]\right)}^{1+\frac{4}{d}} .
$$

Therefore, (2.24) follows from standard continuous argument.

Remark 2.11. Here the restriction on $s$ is different from [27].

As a direct application of $H^{s}$-LWP, we can control the number of LWP intervals covering the interval $\left[t_{k}, t_{k+1}\right]$ as follows.

Lemma 2.12. Let $\frac{1}{1+\min \left\{1, \frac{4}{d}\right\}}<s<1, \frac{4 s}{\min \{4, d\} s-4(1-s)}<\frac{1}{\beta}$. Let $\left\{t_{k}\right\}_{k_{0} \leq k \leq k^{+}}$be defined as in (1.63), and $T^{*} \geq t_{k+1}$. We cover the interval $\left[t_{k}, t_{k+1}\right]$ by LWP time interval $\left\{\tau_{k}^{j}\right\}_{1 \leq j \leq J_{k}}$ given by Corollary [2.10. Then, we have

$$
J_{k} \lesssim k N\left(T^{*}\right)^{\frac{2(1-s)}{s}} .
$$

Proof. First, it follows from (2.20) that

$$
\tau_{k}^{j+1}-\tau_{k}^{j} \sim \frac{1}{\left\|\langle\nabla\rangle I_{N\left(T^{*}\right)} u\left(\tau_{k}^{j}\right)\right\|_{L^{2}}^{\frac{2}{s}}} \gtrsim\left(\frac{1}{N\left(T^{*}\right)^{1-s}\left\|u\left(\tau_{k}^{j}\right)\right\|_{H^{s}}}\right)^{\frac{2}{s}}
$$

This together with Remark 1.14 (1.73) and the almost monotonicity (1.61) implies

$$
\tau_{k}^{j+1}-\tau_{k}^{j} \gtrsim \frac{1}{N\left(T^{*}\right)^{\frac{2(1-s)}{s}}} \lambda\left(\tau_{k}^{j}\right)^{2} \sim \frac{1}{N\left(T^{*}\right)^{\frac{2(1-s)}{s}}} \lambda\left(t_{k}\right)^{2} .
$$

And so (2.26) follows from the control of the blowup speed (1.64).

Lemma 2.13. Let $\left[\tau_{k}^{j}, \tau_{k}^{j+1}\right]$ be a LWP time interval as given by Corollary $[2.10$. Then, there holds:

$$
\begin{aligned}
&\left\|I_{N(t)}\left(|u|^{\frac{4}{d}} u\right)-I_{N(t)} u\left|I_{N(t)} u\right|^{\frac{4}{d}}\right\|_{L_{t}^{2} L_{x}^{\frac{2 d}{d+2}}\left(\left[\tau_{k}^{j}, \tau_{k}^{j+1}\right] \times \mathbb{R}^{d}\right)} \\
& \lesssim \lambda\left(t_{k}\right)^{\frac{1}{\beta} \min \left\{1, \frac{4}{d}\right\} s_{-}}\left\|\langle\nabla\rangle I_{N} u\right\|_{L_{t}^{\infty} L_{x}^{2}\left(\left[\tau_{k}^{j}, \tau_{k}^{j+1}\right] \times \mathbb{R}^{d}\right)}^{\frac{4}{d}} \cdot
\end{aligned}
$$

Proof. we have by triangle inequality

$$
\begin{aligned}
&\left\|\left|I_{N} u\right|^{\frac{4}{d}} I_{N} u-I_{N}\left(|u|^{\frac{4}{d}} u\right)\right\|_{L_{t}^{2} L_{x}^{\frac{2 d}{d+2}}\left(\left[\tau_{k}^{j}, \tau_{k}^{j+1}\right] \times \mathbb{R}^{d}\right)} \\
& \lesssim\left\|I_{N} u\left(\left|I_{N} u\right|^{\frac{4}{d}}-|u|^{\frac{4}{d}}\right)\right\|_{L_{t}^{2} L_{x}^{\frac{2 d}{d+2}}\left(\left[\tau_{k}^{j}, \tau_{k}^{j+1}\right] \times \mathbb{R}^{d}\right)}+\left\|\left(I_{N} u\right)|u|^{\frac{4}{d}}-I_{N}\left(|u|^{\frac{4}{d}} u\right)\right\|_{L_{t}^{2} L_{x}^{\frac{2 d}{d+2}}\left(\left[\tau_{k}^{j}, \tau_{k}^{j+1}\right] \times \mathbb{R}^{d}\right)} .
\end{aligned}
$$


Using (2.10) with $\nu=\min \left\{1, \frac{4}{d}\right\} s-(1-s)_{-}$and (2.13), we estimate

$$
\begin{aligned}
& \left\|\left(I_{N} u\right)|u|^{\frac{4}{d}}-I_{N}\left(|u|^{\frac{4}{d}} u\right)\right\|_{L_{t}^{2} L_{x}^{\frac{2 d}{d+2}}\left(\left[\tau_{k}^{j}, \tau_{k}^{j+1}\right] \times \mathbb{R}^{d}\right)}\left\|\langle\nabla\rangle^{\min \left\{1, \frac{4}{d}\right\} s_{-}}\left(|u|^{\frac{4}{d}}\right)\right\|_{L_{t}^{\infty} L_{x}^{\frac{d}{2}}\left(\left[\tau_{k}^{j}, \tau_{k}^{j+1}\right] \times \mathbb{R}^{d}\right)} \\
\lesssim & \left(t_{k}\right)^{-\min \left\{1, \frac{4}{d}\right\} s_{+}}\left\|I_{N} u\right\|_{L_{t}^{2} L_{x}^{\frac{2 d}{d-2}}\left(\left[\tau_{k}^{j}, \tau_{k}^{j+1}\right] \times \mathbb{R}^{d}\right)}\left\|\left\langle t_{k}\right)^{-\min \left\{1, \frac{4}{d}\right\} s_{+}}\right\| u\left\|_{L_{t}^{\infty} L_{x}^{2}}\right\|\langle\nabla\rangle I_{N} u \|_{L_{t}^{\infty} L_{x}^{2}\left(\left[\tau_{k}^{j}, \tau_{k}^{j+1}\right] \times \mathbb{R}^{d}\right)}^{\frac{4}{d}} \\
\lesssim & \lambda\left(t_{k}\right)^{\frac{1}{\beta} \min \left\{1, \frac{4}{d}\right\} s_{-}}\left\|\langle\nabla\rangle I_{N} u\right\|_{L_{t}^{\infty} L_{x}^{2}\left(\left[\tau_{k}^{j}, \tau_{k}^{j+1}\right] \times \mathbb{R}^{d}\right)} .
\end{aligned}
$$

Similarly,

$$
\begin{aligned}
& \left\|I_{N} u\left(\left|I_{N} u\right|^{\frac{4}{d}}-|u|^{\frac{4}{d}}\right)\right\|_{L_{t}^{2} L_{x}^{\frac{2 d}{d+2}}\left(\left[\tau_{k}^{j}, \tau_{k}^{j+1}\right] \times \mathbb{R}^{d}\right)} \\
& \lesssim\left\|I_{N} u\right\|_{L_{t}^{2} L_{x}^{\frac{2 d}{d-2}}\left(\left[\tau_{k}^{j}, \tau_{k}^{j+1}\right] \times \mathbb{R}^{d}\right)}\left\|\left|I_{N} u-u\right|^{\min \left\{1, \frac{4}{d}\right\}}\left(\left|I_{N} u\right|+|u|\right)^{\frac{4}{d}-\min \left\{1, \frac{4}{d}\right\}}\right\|_{L_{t}^{\infty} L_{x}^{\frac{d}{2}}\left(\left[\tau_{k}^{j}, \tau_{k}^{j+1}\right] \times \mathbb{R}^{d}\right)} \\
& \lesssim\|u\|_{L_{t}^{\infty} L_{x}^{2}}^{1+\frac{4}{d}-\min \left\{1, \frac{4}{d}\right\}}\left\|I_{N} u-u\right\|_{L_{t}^{\infty} L_{x}^{2}\left(\left[\tau_{k}^{j}, \tau_{k}^{j+1}\right] \times \mathbb{R}^{d}\right)}^{\min \left\{1, \frac{4}{4}\right.} \\
& \lesssim N\left(t_{k}\right)^{-\min \left\{1, \frac{4}{d}\right\} s}\left\|\langle\nabla\rangle I_{N} u\right\|_{L_{t}^{\infty} L_{x}^{2}\left(\left[\tau_{k}^{j}, \tau_{k}^{j+1}\right] \times \mathbb{R}^{d}\right)}^{\min \left\{1, \frac{4}{4}\right.}\|u\|_{L_{t}^{\infty} L_{x}^{2}}^{1+\frac{4}{d}-\min \left\{1, \frac{4}{d}\right\}} \\
& \lesssim \lambda\left(t_{k}\right)^{\frac{1}{\beta} \min \left\{1, \frac{4}{d}\right\} s}\left\|\langle\nabla\rangle I_{N} u\right\|_{L_{t}^{\infty} L_{x}^{2}\left(\left[\tau_{k}^{j}, \tau_{k}^{j+1}\right] \times \mathbb{R}^{d}\right)}^{\frac{4}{d}} .
\end{aligned}
$$

2.4. Almost conservation law. First, we consider the increments in the localwellposedness time interval.

Lemma 2.14 (Control of increments in LWP time interval). Let $\frac{1}{1+\min \left\{1, \frac{4}{d}\right\}}<$ $s<1$, $\frac{4 s}{\min \{4, d\} s-4(1-s)}<\frac{1}{\beta}$. Let $\left\{\tau_{k}^{j}\right\}_{1 \leq j \leq J_{k}}$ be the LWP time interval given by Corollary [2.10. Then, for any $T^{*} \geq \tau_{k}^{j+1}$, the modified energy has a slow increment over the LWP time interval:

$$
\begin{aligned}
& \left|E\left(I_{N\left(T^{*}\right)} u\left(\tau_{k}^{j+1}\right)\right)-E\left(I_{N\left(T^{*}\right)} u\left(\tau_{k}^{j}\right)\right)\right| \\
\leq & C N\left(T^{*}\right)^{-\min \left\{1, \frac{4}{d}\right\} s_{+}}\left(\left\|I_{N\left(T^{*}\right)} u\left(\tau_{k}^{j}\right)\right\|_{H^{1}}^{2+\frac{4}{d}}+\left\|I_{N\left(T^{*}\right)} u\left(\tau_{k}^{j}\right)\right\|_{H^{1}}^{2+\frac{8}{d}}\right) .
\end{aligned}
$$

Here, the constant $C$ depends only on s. The modified momentum has a slow increment over the LWP time interval:

$$
\left|P\left(I_{N\left(T^{*}\right)} u\left(\tau_{k}^{j+1}\right)\right)-P\left(I_{N\left(T^{*}\right)} u\left(\tau_{k}^{j}\right)\right)\right| \leq C N\left(T^{*}\right)^{-\min \left\{1, \frac{4}{d}\right\} s_{+}}\left\|I_{N\left(T^{*}\right)} u\left(\tau_{k}^{j}\right)\right\|_{H^{1}}^{2+\frac{4}{d}-\frac{1}{s}} .
$$

Moreover,

$$
\left\|I_{N\left(T^{*}\right)} u\left(\tau_{k}^{j}\right)\right\|_{H^{1}} \leq\left(\frac{N\left(T^{*}\right)}{N\left(\tau_{k}^{j}\right)}\right)^{1-s} \frac{1}{\lambda\left(\tau_{k}^{j}\right)} .
$$

Proof. First, (2.30) follows from Lemma 4.2 in [27] and Corollary 2.10, And (2.32) follows from (3.20) in [5. Thus, we only need to show (2.31). We write $N=N\left(T^{*}\right)$ in this proof. Note that

$$
\operatorname{Re} \int \nabla \Delta I_{N} u \overline{I_{N} u}=0 \text { and } \operatorname{Re} \int \nabla\left(\left|I_{N} u\right|^{\frac{4}{d}} I_{N} u\right) \overline{I_{N} u}=0,
$$


we derive that

$$
\begin{aligned}
\frac{d}{d t} P\left(I_{N} u\right) & =\operatorname{Im} \int \nabla \partial_{t} I_{N} u \overline{I_{N} u}+\operatorname{Im} \int \nabla I_{N} u \overline{\partial_{t} I_{N} u} \\
& =\operatorname{Re} \int\left(\nabla \Delta I_{N} u+\nabla I_{N}\left(|u|^{\frac{4}{d}} u\right)\right) \overline{I_{N} u}-\operatorname{Re} \int \nabla I_{N} u\left(\Delta \overline{I_{N} u}+\overline{I_{N}\left(|u|^{\frac{4}{d}} u\right)}\right) \\
& =2 \operatorname{Re} \int \nabla I_{N}\left(|u|^{\frac{4}{d}} u\right) \overline{I_{N} u} \\
& =2 \operatorname{Re} \int \nabla\left(I_{N}\left(|u|^{\frac{4}{d}} u\right)-\left|I_{N} u\right|^{\frac{4}{d}} I_{N} u\right) \overline{I_{N} u} .
\end{aligned}
$$

Hence,

$$
\begin{aligned}
& \left|P\left(I_{N\left(T^{*}\right)} u\left(\tau_{k}^{j+1}\right)\right)-P\left(I_{N\left(T^{*}\right)} u\left(\tau_{k}^{j}\right)\right)\right| \\
\leq & \left|\int_{\tau_{k}^{j}}^{\tau_{k}^{j+1}} \frac{d}{d t} P\left(I_{N} u\right) d t\right| \\
\lesssim & \int_{\tau_{k}^{j}}^{\tau_{k}^{j+1}} \int_{\mathbb{R}^{d}}\left|\nabla\left(I_{N}\left(|u|^{\frac{4}{d}} u\right)-\left|I_{N} u\right|^{\frac{4}{d}} I_{N} u\right) \overline{I_{N} u}\right| d x d t \\
\lesssim & \left\|\nabla\left[I_{N}\left(|u|^{\frac{4}{d}} u\right)-\left|I_{N} u\right|^{\frac{4}{d}} I_{N} u\right]\right\|_{L_{t}^{2} L_{x}^{\frac{2 d}{d+2}}\left(\left[\tau_{k}^{j}, \tau_{k}^{j+1}\right] \times \mathbb{R}^{d}\right)}\left\|I_{N} u\right\|_{L_{t}^{2} L_{x}^{\frac{2 d}{d-2}}\left(\left[\tau_{k}^{j}, \tau_{k}^{j+1}\right] \times \mathbb{R}^{d}\right)} .
\end{aligned}
$$

It follows from (4.5) in [27] that

$$
\left\|\nabla\left[I_{N}\left(|u|^{\frac{4}{d}} u\right)-\left|I_{N} u\right|^{\frac{4}{d}} I_{N} u\right]\right\|_{L_{t}^{2} L_{x}^{\frac{2 d}{d+2}}\left(\left[\tau_{k}^{j}, \tau_{k}^{j+1}\right] \times \mathbb{R}^{d}\right)} \lesssim N^{-\min \left\{1, \frac{4}{d}\right\} s_{+}}\left\|\langle\nabla\rangle I_{N} u\right\|_{S^{0}\left(\left[\tau_{k}^{j}, \tau_{k}^{j+1}\right]\right)}^{1+\frac{4}{d}},
$$

this together with Hölder's inequality implies that

$$
\begin{aligned}
& \left|P\left(I_{N\left(T^{*}\right)} u\left(\tau_{k}^{j+1}\right)\right)-P\left(I_{N\left(T^{*}\right)} u\left(\tau_{k}^{j}\right)\right)\right| \\
\lesssim & N^{-\min \left\{1, \frac{4}{d}\right\} s_{+}}\left\|\langle\nabla\rangle I_{N} u\right\|_{S^{0}\left(\left[\tau_{k}^{j}, \tau_{k}^{j+1}\right]\right)}^{2+\frac{4}{d}}\left|\tau_{k}^{j}-\tau_{k}^{j+1}\right|^{\frac{1}{2}} \\
\lesssim & N^{-\min \left\{1, \frac{4}{d}\right\} s_{+}}\left\|\langle\nabla\rangle I_{N} u\left(\tau_{k}^{j}\right)\right\|_{L^{2}}^{2+\frac{4}{d}-\frac{1}{s}} .
\end{aligned}
$$

Next, we consider the initial data.

Lemma 2.15. Let

$$
\Xi(t):=\frac{\lambda(t)^{2}}{2} \int\left(|\nabla G(0)|^{2}-\left|\nabla I_{N(t)} G(0)\right|^{2}\right) d x .
$$

Then, we have for $t \in\left[0, T^{+}\right]$,

$$
\begin{gathered}
\left|E\left(I_{N(t)} u(0)\right)+\frac{\Xi(t)}{\lambda(t)^{2}}\right| \lesssim N(t)^{2(1-s)}+\frac{1}{\lambda(t)^{2-\frac{1-\beta}{\beta} \frac{2}{d+2}}}, \\
\left|P\left(I_{N(t)} u(0)\right)\right| \lesssim N(t)^{1-s}+\frac{1}{\lambda(t)^{1-\frac{1-\beta}{2 \beta}}} .
\end{gathered}
$$

Proof. Note that $u_{0}=G(0)+H(0)$, we have

$$
\begin{aligned}
\left|E\left(I_{N(t)} u(0)\right)+\frac{\Xi(t)}{\lambda(t)^{2}}\right| \lesssim \mid & \left|E\left(I_{N(t)} G(0)\right)+\frac{\Xi(t)}{\lambda(t)^{2}}\right| \\
& +\mid E\left(I_{N(t)}(G(0)+H(0))-E\left(I_{N(t)} G(0)\right) \mid .\right.
\end{aligned}
$$


The estimate of (2.36): A simple computation shows

$E\left(I_{N(t)} u(0)\right)+\frac{\Xi(t)}{\lambda(t)^{2}}=E(G(0))+\frac{d}{2(d+2)} \int\left(|G(0)|^{\frac{2(d+2)}{d}}-\left|I_{N(t)} G(0)\right|^{\frac{2(d+2)}{d}}\right) d x$.

Hence, by $G(0) \in H^{1}$ and $\|G(0)\|_{\dot{H}^{s}} \sim \frac{1}{\lambda(0)^{s}}$, (1.44), (1.61) and (1.65), we get

$$
\begin{aligned}
\left|E\left(I_{N(t)} G(0)\right)+\frac{\Xi(t)}{\lambda(t)^{2}}\right| & \leq|E(G(0))|+\left.\frac{d}{2(d+2)} \int|| G(0)\right|^{\frac{2(d+2)}{d}}-\left|I_{N(t)} G(0)\right|^{\frac{2(d+2)}{d}} \mid d x \\
& \lesssim \frac{1}{\sqrt{\lambda(0)}}+\left\|\left(I_{N(t)}-I d\right) G(0)\right\|_{L_{x}^{\frac{2(d+2)}{d}}\|G(0)\|_{L_{x}^{\frac{2(d+2)}{d}}}^{\frac{d+4}{d}}} \\
& \lesssim \frac{1}{\sqrt{\lambda(0)}}+\frac{\left\|\left(I_{N(t)}-I d\right) G(0)\right\|_{\dot{H} \frac{d}{d+2}}}{\lambda(0)^{\frac{d+4}{d+2}}} \\
& \lesssim \frac{1}{\sqrt{\lambda(0)}}+\frac{1}{\lambda(0)^{2}}\left(\frac{1}{\lambda(0) N(t)}\right)^{\frac{2}{d+2}} \\
& \lesssim \frac{1}{\lambda(0)^{2}}\left(\lambda(t)^{\frac{3}{2}}+\lambda(t)^{\frac{1-\beta}{\beta} \frac{2}{d+2}}\right) \\
& \lesssim \frac{1}{\lambda(t)^{2-\frac{1-\beta}{\beta} \frac{2}{d+2}}}
\end{aligned}
$$

The estimate of (2.37): Observe that

$$
\begin{aligned}
|E(u+v)-E(u)| \lesssim & \|\nabla u\|_{L_{x}^{2}}\|\nabla v\|_{L_{x}^{2}}+\|\nabla v\|_{L_{x}^{2}}^{2}+\int\left(|u|^{\frac{d+4}{d}}+|v|^{\frac{d+4}{d}}\right)|v| d x \\
& \lesssim\|\nabla v\|_{L_{x}^{2}}^{2}\left(1+\|v\|_{L_{x}^{2}}^{\frac{4}{d}}\right)+\|\nabla u\|_{L_{x}^{2}}\|\nabla v\|_{L_{x}^{2}} \\
& +\|\nabla u\|_{L^{2}}^{\frac{d+4}{d+2}}\|\nabla v\|_{L^{2}}^{\frac{d}{d+2}}\|u\|_{L^{2}}^{\frac{d+4}{d+2}}\|v\|_{L^{2}}^{\frac{d}{d+2}} .
\end{aligned}
$$

Using (1.36), (1.39), (1.42) and (1.43), we obtain

$$
\begin{gathered}
\left\|\nabla I_{N(t)} G(0)\right\|_{L^{2}} \leq\|\nabla G(0)\|_{L^{2}} \lesssim \frac{1}{\lambda(0)}, \\
\left\|\nabla I_{N(t)} H(0)\right\|_{L^{2}} \lesssim N(t)^{1-s}\|H(0)\|_{H^{s}} \leq \lambda(0)^{10} N(t)^{1-s} .
\end{gathered}
$$

This together with the uniform $L^{2}$ control (1.41) implies

$$
\mid E\left(I_{N(t)}(G(0)+H(0))-E\left(I_{N(t)} G(0)\right) \mid \lesssim N(t)^{2(1-s)} .\right.
$$

And so we obtain (2.34).

Next, we prove (2.35). This part is independent of nonlinear term, so this term is as in [5]. In fact, by (1.45), we have

$$
\begin{aligned}
\left|P\left(I_{N(t)} u(0)\right)\right| \leq & \mid P\left(I_{N(t)}(G(0)+H(0))-P\left(I_{N(t)} G(0)\right) \mid\right. \\
& +\left|P\left(I_{N(t)} G(0)\right)-P(G(0))\right|+|P(G(0))| \\
\lesssim & \mid P\left(I_{N(t)}(G(0)+H(0))-P\left(I_{N(t)} G(0)\right) \mid\right. \\
& +\left|P\left(I_{N(t)} G(0)\right)-P(G(0))\right|+\frac{1}{\sqrt{\lambda(0)}} .
\end{aligned}
$$

A simple computation shows that for $u, v \in \dot{H}^{\frac{1}{2}}$,

$$
|P(u+v)-P(u)| \lesssim\|v\|_{\dot{H}^{\frac{1}{2}}}\left(\|u\|_{\dot{H}^{\frac{1}{2}}}+\|v\|_{\dot{H}^{\frac{1}{2}}}\right) .
$$


Combining this with (2.40) and (2.41), we derive that

$$
\begin{aligned}
& \mid P\left(I_{N(t)}(G(0)+H(0))-P\left(I_{N(t)} G(0)\right) \mid\right. \\
\lesssim & \left\|I_{N(t)} H(0)\right\|_{\dot{H}^{\frac{1}{2}}}\left(\frac{1}{\lambda(0)}+\left\|I_{N(t)} H(0)\right\|_{\dot{H}^{\frac{1}{2}}}\right) \lesssim N(t)^{1-s} .
\end{aligned}
$$

On the other hand,

$$
\begin{aligned}
\left|P\left(I_{N(t)} G(0)\right)-P(G(0))\right| & \lesssim\left\|\left(I d-I_{N(t)}\right) G(0)\right\|_{\dot{H}^{\frac{1}{2}}}\|G(0)\|_{\dot{H}^{\frac{1}{2}}} \\
& \lesssim \frac{1}{\sqrt{\lambda(0)}}\left(\frac{1}{N(t) \lambda(0)}\right)^{\frac{1}{2}} \\
& \lesssim \frac{1}{\sqrt{\lambda(t)}}\left(\frac{1}{N(t) \lambda(t)}\right)^{\frac{1}{2}} \\
& \lesssim \frac{1}{\lambda(t)^{1-\frac{1-\beta}{2 \beta}}} .
\end{aligned}
$$

Proposition 2.16 (Almost conservation laws). Let $\frac{1}{1+\min \left\{1, \frac{4}{d}\right\}}<s<1$, $\frac{4 s}{\min \{4, d\} s-4(1-s)}<$ $\frac{1}{\beta}$. There holds the following control of the modified energy and momentum on $\left[0, T^{+}\right]$:

$$
\begin{aligned}
\left|E\left(I_{N(t)} u(t)\right)+\frac{\Xi(t)}{\lambda(t)^{2}}\right| & \leq \frac{1}{\lambda(t)^{2\left(1-\alpha_{1}\right)}}, \\
\left|P\left(I_{N(t)} u(t)\right)\right| & \leq \frac{1}{\lambda(t)^{1-\alpha_{1}}},
\end{aligned}
$$

for some $\alpha_{1}=\frac{1-\beta}{4 \beta}$. In other words,

$$
\begin{aligned}
\left|\lambda(t)^{2} E\left(I_{N(t)} u(t)\right)+\Xi(t)\right| & \leq \lambda(t)^{2 \alpha_{1}} \leq \Gamma_{b(t)}^{10}, \\
\lambda(t)\left|P\left(I_{N(t)} u(t)\right)\right| & \leq \lambda(t)^{\alpha_{1}} \leq \Gamma_{b(t)}^{10} .
\end{aligned}
$$

Proof. Without loss of generality, we may assume $t=T^{+}$. By (2.30), (2.26), (2.34), (2.32), we have

$$
\begin{aligned}
& \quad\left|E\left(I_{N\left(T^{+}\right)} u\left(T^{+}\right)\right)+\frac{\Xi\left(T^{+}\right)}{\lambda\left(T^{+}\right)^{2}}\right| \\
& \leq\left|E\left(I_{N\left(T^{+}\right)} u(0)\right)+\frac{\Xi\left(T^{+}\right)}{\lambda\left(T^{+}\right)^{2}}\right|+\sum_{k=k_{0}} \sum_{j=1}^{k_{k}}\left|E\left(I_{N\left(T^{+}\right)} u\left(\tau_{k}^{j+1}\right)\right)-E\left(I_{N\left(T^{+}\right)} u\left(\tau_{k}^{j}\right)\right)\right| \\
& \lesssim N\left(T^{+}\right)^{2(1-s)}+\frac{1}{\lambda\left(T^{+}\right)^{2-\frac{1-\beta}{\beta} \frac{2}{d+2}}} \\
& \quad+\sum_{k=k_{0}}^{k^{+}} k N\left(T^{+}\right)^{\frac{2(1-s)}{s}} N\left(T^{+}\right)^{-\min \left\{1, \frac{4}{d}\right\} s_{+}}\left(\left\|I_{N\left(T^{+}\right)} u\left(\tau_{k}^{j}\right)\right\|_{H^{1}}^{2+\frac{4}{d}}+\left\|I_{N\left(T^{+}\right)} u\left(\tau_{k}^{j}\right)\right\|_{H^{1}}^{2+\frac{8}{d}}\right) \\
& \lesssim\left(\frac{1}{\lambda\left(T^{+}\right)}\right)^{\frac{2(1-s)}{\beta}}+\frac{1}{\lambda\left(T^{+}\right)^{2-\frac{1-\beta}{\beta}} \frac{2}{d+2}} \\
& \quad+\sum_{k=k_{0}}^{k^{+}} k N\left(T^{+}\right)^{\frac{2(1-s)}{s}} N\left(T^{+}\right)^{-\min \left\{1, \frac{4}{d}\right\} s_{+}}\left[\left(\frac{N\left(T^{+}\right)}{N\left(t_{k}\right)}\right)^{1-s} \frac{1}{\lambda\left(t_{k}\right)}\right]^{2+\frac{8}{d}} .
\end{aligned}
$$


We now sum up the geometric series from (1.63) to get

$$
\begin{aligned}
& \sum_{k=k_{0}}^{k^{+}} k N\left(T^{+}\right)^{\frac{2(1-s)}{s}} N\left(T^{+}\right)^{-\min \left\{1, \frac{4}{d}\right\} s_{+}}\left[\left(\frac{N\left(T^{+}\right)}{N\left(t_{k}\right)}\right)^{1-s} \frac{1}{\lambda\left(t_{k}\right)}\right]^{2+\frac{8}{d}} \\
\lesssim & N\left(T^{+}\right)^{(1-s)\left(2+\frac{8}{d}+\frac{2}{s}\right)-\min \left\{1, \frac{4}{d}\right\} s_{+}} \sum_{k=k_{0}}^{k^{+}} k N\left(t_{k}\right)^{\left(2+\frac{8}{d}\right)(\beta-(1-s))} \\
\lesssim & k^{+} N\left(T^{+}\right)^{\left(2+\frac{8}{d}\right) \beta+\frac{2(1-s)}{s}-\min \left\{1, \frac{4}{d}\right\} s_{+}} \\
\lesssim & \log \left(\lambda\left(T^{+}\right)\right) \mid\left(\frac{1}{\lambda\left(T^{+}\right)}\right)^{\frac{1}{\beta}\left(\left(2+\frac{8}{d}\right) \beta+\frac{2(1-s)}{s}-\min \left\{1, \frac{4}{d}\right\} s_{+}\right)} \\
\lesssim & \left(\frac{1}{\lambda\left(T^{+}\right)}\right)^{\frac{1}{\beta}\left(\left(2+\frac{8}{d}\right) \beta+\frac{2(1-s)}{s}-\min \left\{1, \frac{4}{d}\right\}_{+}\right)} .
\end{aligned}
$$

Similarly, using (2.31), (2.26), (2.35) and (2.32), we estimate

$$
\begin{aligned}
& \left|P\left(I_{N\left(T^{+}\right)} u\left(T^{+}\right)\right)\right| \\
\leq & \left|P\left(I_{N\left(T^{+}\right)} u(0)\right)\right|+\sum_{k=k_{0}}^{k^{+}} \sum_{j=1}^{J_{k}}\left|P\left(I_{N\left(T^{+}\right)} u\left(\tau_{k}^{j+1}\right)\right)-P\left(I_{N\left(T^{+}\right)} u\left(\tau_{k}^{j}\right)\right)\right| \\
\lesssim & \left(T^{+}\right)^{1-s}+\frac{1}{\lambda\left(T^{+}\right)^{1-\frac{1-\beta}{2 \beta}}}+\sum_{k=k_{0}}^{k^{+}} k N\left(T^{+}\right)^{\frac{2(1-s)}{s}} N\left(T^{+}\right)^{-\min \left\{1, \frac{4}{d}\right\} s_{+}}\left\|I_{N\left(T^{+}\right)} u\left(t_{k}\right)\right\|_{H^{1}}^{1+\frac{4}{d}} \\
\lesssim & \left(\frac{1}{\lambda\left(T^{+}\right)}\right)^{\frac{1-s}{\beta}}+\frac{1}{\lambda\left(T^{+}\right)^{1-\frac{1-\beta}{2 \beta}}}+\sum_{k=k_{0}}^{k^{+}} k N\left(T^{+}\right)^{\frac{2(1-s)}{s}}-\min \left\{1, \frac{4}{d}\right\} s_{+}\left[\left(\frac{N\left(T^{+}\right)}{N\left(t_{k}\right)}\right)^{1-s} \frac{1}{\lambda\left(t_{k}\right)}\right]^{2+\frac{4}{d}-\frac{1}{s}} \\
\lesssim & \left(\frac{1}{\lambda\left(T^{+}\right)}\right)^{\frac{1-s}{\beta}}+\frac{1}{\lambda\left(T^{+}\right)^{1-\frac{1-\beta}{2 \beta}}}+\left(\frac{1}{\lambda\left(T^{+}\right)}\right)^{\frac{1}{\beta}\left(\left(2+\frac{8}{d}-\frac{1}{s}\right) \beta+\frac{2(1-s)}{s}-\min \left\{1, \frac{4}{d}\right\} s_{+}\right)} .
\end{aligned}
$$

\section{Proof of Proposition 1.9}

In this section, we will show Proposition 1.9 and then we conclude the proof of our main Theorem 1.4

3.1. Control of the geometrical parameters. Recall the geometrical decoposition

$$
u(t, x)=\frac{1}{\lambda(t)^{\frac{d}{2}}}\left(Q_{b(t)}(\cdot)+\varepsilon(t, \cdot)\right)\left(\frac{x-x(t)}{\lambda(t)}\right) e^{-i \gamma(t)}, t \in\left[0, T^{+}\right] .
$$

Let us introduce the rescaled time

$$
\frac{d s}{d t}=\frac{1}{\lambda(s)^{2}} \quad \text { with } \quad s(0)=s_{0}=e^{\frac{5 \pi}{9 b(0)}}
$$

and $y=\frac{x-x(t)}{\lambda(t)}$. Then, $\varepsilon(s, y)$ satisfies on $\left[0, T^{+}\right]$the equation:

$$
\begin{aligned}
& i \frac{\partial Q_{b}}{\partial b} b_{s}+i \partial_{s} \varepsilon+\Delta Q_{b}+\Delta \varepsilon+\left|Q_{b}+\varepsilon\right|^{\frac{4}{d}}\left(Q_{b}+\varepsilon\right) \\
= & -\left(\gamma_{s}+i \frac{d}{2} \frac{\lambda_{s}}{\lambda}\right)\left(Q_{b}+\varepsilon\right)+i\left(\frac{x_{s}}{\lambda}+\frac{\lambda_{s}}{\lambda} y\right) \cdot \nabla\left(Q_{b}+\varepsilon\right) .
\end{aligned}
$$


To simplify notations, we note

$$
\varepsilon=\varepsilon_{1}+i \varepsilon_{2}, Q_{b}=\Sigma+i \Theta
$$

in terms of real and imaginary parts. We have by using Remark 1.7

$$
\begin{aligned}
b_{s} \frac{\partial \Sigma}{\partial b}+\partial_{s} \varepsilon_{1}-M_{-}(\varepsilon)+b \Lambda \varepsilon_{1}= & \left(\frac{\lambda_{s}}{\lambda}+b\right) \Lambda \Sigma+\tilde{\gamma}_{s} \Theta+\frac{x_{s}}{\lambda} \cdot \nabla \Sigma \\
& +\left(\frac{\lambda_{s}}{\lambda}+b\right) \Lambda \varepsilon_{1}+\tilde{\gamma}_{s} \varepsilon_{2}+\frac{x_{s}}{\lambda} \cdot \nabla \varepsilon_{1} \\
& +\operatorname{Im}\left(\Psi_{b}\right)-R_{2}(\varepsilon) \\
b_{s} \frac{\partial \Theta}{\partial b}+\partial_{s} \varepsilon_{2}+M_{+}(\varepsilon)+b \Lambda \varepsilon_{2}= & \left(\frac{\lambda_{s}}{\lambda}+b\right) \Lambda \Theta-\tilde{\gamma}_{s} \Sigma+\frac{x_{s}}{\lambda} \cdot \nabla \Theta \\
& +\left(\frac{\lambda_{s}}{\lambda}+b\right) \Lambda \varepsilon_{2}-\tilde{\gamma}_{s} \varepsilon_{1}+\frac{x_{s}}{\lambda} \cdot \nabla \varepsilon_{2} \\
& -\operatorname{Re}\left(\Psi_{b}\right)+R_{1}(\varepsilon),
\end{aligned}
$$

with $\tilde{\gamma}(s)=-s-\gamma(s)$. The linear operator close to $Q_{b}$ is now a deformation of the linear operator $L$ close to $Q$ and is $M=\left(M_{+}, M_{-}\right)$with

$$
\begin{aligned}
& M_{+}(\varepsilon)=-\Delta \varepsilon_{1}+\varepsilon_{1}-\left(\frac{4 \Sigma^{2}}{d\left|Q_{b}\right|^{2}}+1\right)\left|Q_{b}\right|^{\frac{4}{d}} \varepsilon_{1}-\left(\frac{4 \Sigma \Theta}{d\left|Q_{b}\right|^{2}}\left|Q_{b}\right|^{\frac{4}{d}}\right) \varepsilon_{2} \\
& M_{-}(\varepsilon)=-\Delta \varepsilon_{2}+\varepsilon_{2}-\left(\frac{4 \Theta^{2}}{d\left|Q_{b}\right|^{2}}+1\right)\left|Q_{b}\right|^{\frac{4}{d}} \varepsilon_{2}-\left(\frac{4 \Sigma \Theta}{d\left|Q_{b}\right|^{2}}\left|Q_{b}\right|^{\frac{4}{d}}\right) \varepsilon_{1} .
\end{aligned}
$$

The formally quadratic in $\varepsilon$ interaction terms are:

$$
\begin{aligned}
& R_{1}(\varepsilon)=\left(\varepsilon_{1}+\Sigma\right)\left|\varepsilon+Q_{b}\right|^{\frac{4}{d}}-\Sigma\left|Q_{b}\right|^{\frac{4}{d}}-\left(\frac{4 \Sigma^{2}}{d\left|Q_{b}\right|^{2}}+1\right)\left|Q_{b}\right|^{\frac{4}{d}} \varepsilon_{1}-\left(\frac{4 \Sigma \Theta}{d\left|Q_{b}\right|^{2}}\left|Q_{b}\right|^{\frac{4}{d}}\right) \varepsilon_{2}, \\
& R_{2}(\varepsilon)=\left(\varepsilon_{2}+\Theta\right)\left|\varepsilon+Q_{b}\right|^{\frac{4}{d}}-\Theta\left|Q_{b}\right|^{\frac{4}{d}}-\left(\frac{4 \Theta^{2}}{d\left|Q_{b}\right|^{2}}+1\right)\left|Q_{b}\right|^{\frac{4}{d}} \varepsilon_{2}-\left(\frac{4 \Sigma \Theta}{d\left|Q_{b}\right|^{2}}\left|Q_{b}\right|^{\frac{4}{d}}\right) \varepsilon_{1} .
\end{aligned}
$$

The formally cubic terms in $\epsilon$ are:

$$
\begin{aligned}
\widetilde{R}_{1}(\epsilon) & =R_{1}(\epsilon)-\epsilon_{1}^{2} \frac{\left|Q_{b}\right|^{\frac{4}{d}}}{\left|Q_{b}\right|^{4}}\left[\frac{2}{d}\left(1+\frac{4}{d}\right) \Sigma^{3}+\frac{6}{d} \Sigma \Theta^{2}\right] \\
& -\epsilon_{2}^{2} \frac{\left|Q_{b}\right|^{\frac{4}{d}}}{\left|Q_{b}\right|^{4}}\left[\frac{2}{d} \Sigma^{3}+\frac{2}{d}\left(\frac{4}{d}-1\right) \Sigma \Theta^{2}\right] \\
& -\frac{4}{d} \frac{\left|Q_{b}\right|^{\frac{4}{d}}}{\left|Q_{b}\right|^{4}} \epsilon_{1} \epsilon_{2}\left[\left(\frac{4}{d}-1\right) \Sigma^{2} \Theta+\Theta^{3}\right] \\
\widetilde{R}_{2}(\epsilon) & =R_{2}(\epsilon)-\epsilon_{2}^{2} \frac{\left|Q_{b}\right|^{\frac{4}{d}}}{\left|Q_{b}\right|^{4}}\left[\frac{2}{d}\left(1+\frac{4}{d}\right) \Theta^{3}+\frac{6}{d} \Theta \Sigma^{2}\right] \\
& -\epsilon_{1}^{2} \frac{\left|Q_{b}\right|^{\frac{4}{d}}}{\left|Q_{b}\right|^{4}}\left[\frac{2}{d} \Theta^{3}+\frac{2}{d}\left(\frac{4}{d}-1\right) \Theta \Sigma^{2}\right] \\
& -\frac{4}{d} \frac{\left|Q_{b}\right|^{\frac{4}{d}}}{\left|Q_{b}\right|^{4}} \epsilon_{1} \epsilon_{2}\left[\left(\frac{4}{d}-1\right) \Theta^{2} \Sigma+\Sigma^{3}\right]
\end{aligned}
$$

Remark 3.1. From Weinstein 29, the linearized operator $L$ close to the ground state in dimension $d$ can be explicitly written $L=\left(L_{+}, L_{-}\right)$with

$$
L_{+}=-\Delta+1-\left(\frac{4}{d}+1\right) Q^{\frac{4}{d}}, \quad L_{-}=-\Delta+1-Q^{\frac{4}{d}},
$$


and the following algebraic relations hold:

$$
\begin{gathered}
L_{+}(\Lambda Q)=-2 Q, L_{+}(\nabla Q)=0, \\
L_{-}(Q)=0, L_{-}(y Q)=-2 \nabla Q, L_{-}\left(|y|^{2} Q\right)=-4 \Lambda Q .
\end{gathered}
$$

Recall

$$
\left\|e^{C r}\left(Q_{b}-Q\right)\right\|_{H^{10} \cap C^{2}} \rightarrow 0 \quad \text { as } \quad|b| \rightarrow 0,
$$

we can replace $Q_{b(s)}$ by $Q$ in the following with some loss such as $\Gamma_{b(s)}^{10}$.

Lemma 3.2 (Estimates induced by conservation laws). For all $s \in\left[s_{0}, s^{+}\right]$with $s^{+}=s\left(T^{+}\right)$, there holds:

$$
\begin{aligned}
& \left.\quad\left|\left[2\left(\varepsilon_{1}, \Sigma+b \Lambda \Theta-\operatorname{Re}\left(\Psi_{b}\right)\right)+2\left(\varepsilon_{2}, \Theta-b \Lambda \Sigma-\operatorname{Im}\left(\Psi_{b}\right)\right)\right]-2 \Xi(s)-\int\right| I_{N(s) \lambda(s)} \nabla \varepsilon(s)\right|^{2} \\
& \quad+\left[\int\left(\frac{4}{d}+1\right)|Q|^{\frac{4}{d}}\left(I_{N \lambda} \varepsilon_{1}\right)^{2}+\int|Q|^{\frac{4}{d}}\left(I_{N \lambda} \varepsilon_{2}\right)^{2}\right] \mid \\
& \leq \delta_{0}\left(\int\left|I_{N(s) \lambda(s)} \nabla \varepsilon(s)\right|^{2}+\int|\varepsilon(s)|^{2} e^{-|y|}\right)+\Gamma_{b(s)}^{1-C \eta}
\end{aligned}
$$

and

$$
\left|\left(\varepsilon_{2}, \nabla Q\right)\right| \leq \delta_{0}\left(\int\left|I_{N(s) \lambda(s)} \nabla \varepsilon(s)\right|^{2}\right)^{\frac{1}{2}}+\Gamma_{b(s)}^{10} .
$$

Proof. Note that

$$
I_{N} u(t, x)=\frac{1}{\lambda^{\frac{d}{2}}}\left[I_{N \lambda}\left(Q_{b}+\varepsilon\right)\right]\left(\frac{x-x(t)}{\lambda(t)}\right)
$$

we have

$$
\begin{aligned}
2 \lambda^{2} E\left(I_{N} u\right)= & 2 E\left(I_{N \lambda}\left(Q_{b}+\varepsilon\right)\right) \\
= & 2 E\left(I_{N \lambda}\left(Q_{b}+\varepsilon\right)\right)-2 E\left(Q_{b}+I_{N \lambda} \varepsilon\right) \\
& +2 E\left(Q_{b}+I_{N \lambda} \varepsilon\right) .
\end{aligned}
$$

On the other hand, observe that

$$
\Delta Q_{b}-Q_{b}+i b \Lambda Q_{b}+\left|Q_{b}\right|^{\frac{4}{d}} Q_{b}=-\Psi_{b}
$$

we get

$$
\begin{aligned}
& 2 E\left(Q_{b}+I_{N \lambda} \varepsilon\right)=\int\left|\nabla\left(Q_{b}+I_{N \lambda} \varepsilon\right)\right|^{2}-\frac{d}{d+2} \int\left|Q_{b}+I_{N \lambda} \varepsilon\right|^{\frac{2(d+2)}{d}} \\
= & \int\left(\left|\nabla Q_{b}\right|^{2}-2 \operatorname{Re}\left(\Delta Q_{b} \cdot \overline{I_{N \lambda} \varepsilon}\right)+\left|\nabla I_{N \lambda} \varepsilon\right|^{2}\right)-\frac{d}{d+2} \int\left|Q_{b}+I_{N \lambda} \varepsilon\right|^{\frac{2(d+2)}{d}} \\
= & 2 E\left(Q_{b}\right)+\int\left|\nabla I_{N \lambda} \varepsilon\right|^{2}-2 \operatorname{Re} \int\left(\Delta Q_{b}+\left|Q_{b}\right|^{\frac{4}{d}} Q_{b}\right) \overline{I_{N \lambda} \varepsilon} \\
& -\frac{d}{d+2} \int\left(\left|Q_{b}+I_{N \lambda} \varepsilon\right|^{\frac{2(d+2)}{d}}-\left|Q_{b}\right|^{\frac{2(d+2)}{d}}-\frac{2(d+2)}{d}\left|Q_{b}\right|^{\frac{4}{d}} \operatorname{Re}\left(Q_{b} \overline{I_{N \lambda} \varepsilon}\right)\right)
\end{aligned}
$$


and

$$
\begin{aligned}
& 2 \operatorname{Re} \int\left(\Delta Q_{b}+\left|Q_{b}\right|^{\frac{4}{d}} Q_{b}\right) \overline{I_{N \lambda} \varepsilon}=2 \operatorname{Re} \int\left(Q_{b}-i b \Lambda Q_{b}-\Psi_{b}\right) \overline{I_{N \lambda} \varepsilon} \\
= & 2 \operatorname{Re} \int\left(Q_{b}-i b \Lambda Q_{b}-\Psi_{b}\right) \bar{\varepsilon}+2 \operatorname{Re} \int\left(I_{N \lambda}-\operatorname{Id}\right)\left(Q_{b}-i b \Lambda Q_{b}-\Psi_{b}\right) \bar{\varepsilon} \\
= & 2\left(\varepsilon_{1}, \Sigma+b \Lambda \Theta-\operatorname{Re}\left(\Psi_{b}\right)\right)+2\left(\varepsilon_{2}, \Theta-b \Lambda \Sigma-\operatorname{Im}\left(\Psi_{b}\right)\right) \\
& +2 \operatorname{Re} \int\left(I_{N \lambda}-\mathrm{Id}\right)\left(Q_{b}-i b \Lambda Q_{b}-\Psi_{b}\right) \bar{\varepsilon} .
\end{aligned}
$$

Hence,

$$
\begin{aligned}
& 2\left(\varepsilon_{1}, \Sigma+b \Lambda \Theta-\operatorname{Re}\left(\Psi_{b}\right)\right)+2\left(\varepsilon_{2}, \Theta-b \Lambda \Sigma-\operatorname{Im}\left(\Psi_{b}\right)\right)-2 \Xi(s)-\int\left|I_{N(s) \lambda(s)} \nabla \varepsilon(s)\right|^{2} \\
& +\int\left(\frac{4 \Sigma^{2}}{d\left|Q_{b}\right|^{2}}+1\right)\left|Q_{b}\right|^{\frac{4}{d}}\left(I_{N \lambda} \varepsilon_{1}\right)^{2}+\int\left(\frac{4 \Theta^{2}}{d\left|Q_{b}\right|^{2}}+1\right)\left|Q_{b}\right|^{\frac{4}{d}}\left(I_{N \lambda} \varepsilon_{2}\right)^{2} \\
= & 2 E\left(Q_{b}\right)-2\left(\lambda^{2} E\left(I_{N} u\right)+\Xi(s)\right)+2 E\left(I_{N \lambda}\left(Q_{b}+\varepsilon\right)\right)-2 E\left(Q_{b}+I_{N \lambda} \varepsilon\right) \\
& -2 \operatorname{Re} \int\left(I_{N \lambda}-\mathrm{Id}\right)\left(Q_{b}-i b \Lambda Q_{b}-\Psi_{b}\right) \bar{\varepsilon} \\
& -8 \int \frac{\Sigma \Theta}{d\left|Q_{b}\right|^{2}}\left|Q_{b}\right|^{\frac{4}{d}} I_{N \lambda} \varepsilon_{1} I_{N \lambda} \varepsilon_{2}-\frac{d}{d+2} \int J\left(I_{N \lambda} \varepsilon\right),
\end{aligned}
$$

where the cubic term $J(\varepsilon)$ ( is given in Appendix B in [20])

$$
\begin{aligned}
J(\varepsilon)= & \left|\varepsilon+Q_{b}\right|^{2+\frac{4}{d}}-\left|Q_{b}\right|^{2+\frac{4}{d}}-\left(2+\frac{4}{d}\right)\left|Q_{b}\right|^{\frac{4}{d}}\left(\Sigma \varepsilon_{1}+\Theta \varepsilon_{2}\right) \\
& -\varepsilon_{1}^{2}\left|Q_{b}\right|^{\frac{4}{d}-2}\left[\left(1+\frac{2}{d}\right)\left(1+\frac{4}{d}\right) \Sigma^{2}+\left(1+\frac{2}{d}\right) \Theta^{2}\right] \\
& -\varepsilon_{2}^{2}\left|Q_{b}\right|^{\frac{4}{d}-2}\left[\left(1+\frac{2}{d}\right)\left(1+\frac{4}{d}\right) \Theta^{2}+\left(1+\frac{2}{d}\right) \Sigma^{2}\right] \\
& -\varepsilon_{1} \varepsilon_{2}\left|Q_{b}\right|^{\frac{4}{d}-2} \frac{8}{d}\left(1+\frac{2}{d}\right) \Sigma \Theta .
\end{aligned}
$$

Indeed, $J(\epsilon)$ is the formal cubic term in $\epsilon$ in the expansion

$$
\left|\epsilon+Q_{b}\right|^{2+\frac{4}{d}}=\left|Q_{b}\right|^{2+\frac{4}{d}}\left[1+\frac{2\left(\Sigma \epsilon_{1}+\Theta \epsilon_{2}\right)}{\left|Q_{b}\right|^{2}}+\frac{|\epsilon|^{2}}{\left|Q_{b}\right|^{2}}\right]^{\frac{d+2}{d}} .
$$

In fact, $J(\epsilon)$ is not cubic in $\epsilon$ when $d \geq 4$, but from elementary inequality, $J(\epsilon)=$ $O\left(|\epsilon|^{2+\frac{4}{d}}\right)$. This can give us the desired smallness in the sequel.

Then, (3.5) follows by (1.29), Proposition 2.16] and

$$
\left\|\left(I_{N(t) \lambda(t)}-\mathrm{Id}\right) Q\right\|_{H^{p}} \lesssim \lambda(t)^{C_{p}} \lesssim \Gamma_{b(t)}^{10} .
$$

Next, we turn to prove (3.6). Observe that

$$
\begin{aligned}
\lambda P\left(I_{N} u\right) & =P\left(I_{N \lambda}\left(Q_{b}+\varepsilon\right)\right) \\
& =\left(P\left(I_{N \lambda}\left(Q_{b}+\varepsilon\right)\right)-P\left(Q_{b}+I_{N \lambda} \varepsilon\right)\right)+P\left(Q_{b}+I_{N \lambda} \varepsilon\right)
\end{aligned}
$$


and

$$
\begin{aligned}
P\left(Q_{b}+I_{N \lambda} \varepsilon\right)= & \operatorname{Im} \int \nabla\left(Q_{b}+I_{N \lambda} \varepsilon\right)\left(\overline{Q_{b}+I_{N \lambda} \varepsilon}\right) \\
= & -2 \int I_{N \lambda} \varepsilon_{2} \nabla \Sigma-2 \int \Theta \nabla \Sigma+2 \int I_{N \lambda} \varepsilon_{1} \nabla \Theta+2 \int I_{N \lambda} \varepsilon_{1} \nabla I_{N \lambda} \varepsilon_{2} \\
= & -2\left(\varepsilon_{2}, \nabla Q\right)+2\left(\varepsilon_{2}, \nabla(Q-\Sigma)\right)+2\left(\varepsilon_{2},\left(\mathrm{Id}-I_{N \lambda}\right) \nabla \Sigma\right) \\
& -2 \int \Theta \nabla \Sigma+2 \int I_{N \lambda} \varepsilon_{1} \nabla \Theta+2 \int I_{N \lambda} \varepsilon_{1} \nabla I_{N \lambda} \varepsilon_{2},
\end{aligned}
$$

then, we obtain

$$
\begin{aligned}
-2\left(\varepsilon_{2}, \nabla Q\right)= & \lambda P\left(I_{N} u\right)-\left(P\left(I_{N \lambda}\left(Q_{b}+\varepsilon\right)\right)-P\left(Q_{b}+I_{N \lambda} \varepsilon\right)\right) \\
& -2\left(\varepsilon_{2}, \nabla(Q-\Sigma)\right)-2\left(\varepsilon_{2},\left(\mathrm{Id}-I_{N \lambda}\right) \nabla \Sigma\right) \\
& +2 \int \Theta \nabla \Sigma-2 \int I_{N \lambda} \varepsilon_{1} \nabla \Theta-2 \int I_{N \lambda} \varepsilon_{1} \nabla I_{N \lambda} \varepsilon_{2} .
\end{aligned}
$$

Note that $(\Theta, \nabla \Sigma)=0$ since $Q_{b}$ is radial. Thus, we get (3.6) by (1.27) and Proposition 2.16. Therefore, we conclude the proof of Lemma 3.2

Recall

$$
\left.\left(Q_{b}\right)\right|_{b=0}=Q,\left.\quad\left(\frac{\partial Q_{b}}{\partial b}\right)\right|_{b=0}=-i \frac{|y|^{2}}{4} Q .
$$

It is easy to see that

$$
\nabla \Lambda f-\Lambda \nabla f=\nabla f,(f, \Lambda g)=-(\Lambda f, g) .
$$

Lemma 3.3. There holds

(i)

$$
\begin{aligned}
& b_{s}\left[\left(\frac{\partial \Theta}{\partial b}, \Lambda \Sigma\right)-\left(\frac{\partial \Sigma}{\partial b}, \Lambda \Theta\right)+\left(\varepsilon_{1}, \frac{\partial \Lambda \Theta}{\partial b}\right)-\left(\varepsilon_{2}, \frac{\partial \Lambda \Sigma}{\partial b}\right)\right] \\
= & -\left(M_{+}(\varepsilon), \Lambda \Sigma\right)-\left(M_{-}(\varepsilon), \Lambda \Theta\right)-\tilde{\gamma}_{s}\left[\left(\varepsilon_{1}, \Lambda \Sigma\right)+\left(\varepsilon_{2}, \Lambda \Theta\right)\right] \\
& -\frac{x_{s}}{\lambda} \cdot\left[(\nabla \Sigma, \Lambda \Theta)-(\nabla \Theta, \Lambda \Sigma)+\left(\varepsilon_{2}, \nabla \Lambda \Sigma\right)-\left(\varepsilon_{1}, \nabla \Lambda \Theta\right)\right] \\
& +\left(R_{1}(\varepsilon), \Lambda \Sigma\right)+\left(R_{2}(\varepsilon), \Lambda \Theta\right)-\left(\operatorname{Re}\left(\Psi_{b}\right), \Lambda \Sigma\right)-\left(\operatorname{Im}\left(\Psi_{b}\right), \Lambda \Theta\right),
\end{aligned}
$$

(ii)

$$
\begin{aligned}
& \left(\frac{\lambda_{s}}{\lambda}+b\right)\left[\left(\Lambda \Sigma,|y|^{2} \Sigma\right)+\left(\Lambda \Theta,|y|^{2} \Theta\right)\right] \\
= & \left(M_{+}(\varepsilon),|y|^{2} \Theta\right)-\left(M_{-}(\varepsilon),|y|^{2} \Sigma\right)-\frac{\lambda_{s}}{\lambda}\left[\left(\varepsilon_{1}, \Lambda\left(|y|^{2} \Sigma\right)\right)+\left(\varepsilon_{2}, \Lambda\left(|y|^{2} \Theta\right)\right)\right] \\
& +b_{s}\left[\left(\frac{\partial \Sigma}{\partial b},|y|^{2} \Sigma\right)+\left(\frac{\partial \Theta}{\partial b},|y|^{2} \Theta\right)-\left(\varepsilon_{1}, \frac{\partial\left(|y|^{2} \Sigma\right)}{\partial b}\right)-\left(\varepsilon_{2}, \frac{\partial\left(|y|^{2} \Theta\right)}{\partial b}\right)\right] \\
& +\tilde{\gamma}_{s}\left[\left(\varepsilon_{1},|y|^{2} \Theta\right)-\left(\varepsilon_{2},|y|^{2} \Sigma\right)\right]+\frac{x_{s}}{\lambda} \cdot\left[\left(\varepsilon_{1},|y|^{2} \nabla \Sigma\right)+\left(\varepsilon_{2},|y|^{2} \nabla \Theta\right)\right] \\
& -\left(R_{1}(\varepsilon),|y|^{2} \Theta\right)+\left(R_{2}(\varepsilon),|y|^{2} \Sigma\right)-\left(\operatorname{Im}\left(\Psi_{b}\right),|y|^{2} \Sigma\right)+\left(\operatorname{Re}\left(\Psi_{b}\right),|y|^{2} \Theta\right),
\end{aligned}
$$


(iii)

$$
\begin{aligned}
& \frac{x_{s}}{\lambda} \cdot\left[(\nabla \Sigma, y \Sigma)+(\nabla \Theta, y \Theta)-\left(\varepsilon_{1}, \nabla(y \Sigma)\right)-\left(\varepsilon_{2}, \nabla(y \Theta)\right)\right] \\
= & \left(M_{+}(\varepsilon), y \Theta\right)-\left(M_{-}(\varepsilon), y \Sigma\right)+\tilde{\gamma}_{s}\left[\left(\varepsilon_{1}, y \Theta\right)-\left(\varepsilon_{2}, y \Sigma\right)\right] \\
& -b_{s}\left[\left(\varepsilon_{1}, y \frac{\partial \Sigma}{\partial b}\right)+\left(\varepsilon_{2}, y \frac{\partial \Theta}{\partial b}\right)\right]+\frac{\lambda_{s}}{\lambda}\left[\left(\varepsilon_{1}, \Lambda(y \Sigma)\right)+\left(\varepsilon_{2}, \Lambda(y \Theta)\right)\right] \\
& -\left(R_{1}(\varepsilon), y \Theta\right)+\left(R_{2}(\varepsilon), y \Sigma\right)-\left(\operatorname{Im}\left(\Psi_{b}\right), y \Sigma\right)+\left(\operatorname{Re}\left(\Psi_{b}\right), y \Theta\right),
\end{aligned}
$$

$(i v)$

$$
\begin{aligned}
& \tilde{\gamma}_{s}\left[\left(\Theta, \Lambda^{2} \Theta\right)+\left(\Sigma, \Lambda^{2} \Sigma\right)+\left(\varepsilon_{1}, \Lambda^{2} \Sigma\right)+\left(\varepsilon_{2}, \Lambda^{2} \Theta\right)\right] \\
= & \left(M_{+}(\varepsilon), \Lambda^{2} \Sigma\right)+\left(M_{-}(\varepsilon), \Lambda^{2} \Theta\right)+\tilde{\gamma}_{s}\left[\left(\varepsilon_{1}, \Lambda^{2} \Sigma\right)+\left(\varepsilon_{2}, \Lambda^{2} \Theta\right)\right] \\
& -b_{s}\left[\left(\frac{\partial \Sigma}{\partial b}, \Lambda^{2} \Theta\right)-\left(\frac{\partial \Theta}{\partial b}, \Lambda^{2} \Sigma\right)-\left(\varepsilon_{1}, \frac{\partial \Lambda^{2} \Theta}{\partial b}\right)+\left(\varepsilon_{2}, \frac{\partial \Lambda^{2} \Sigma}{\partial b}\right)\right] \\
& +\frac{x_{s}}{\lambda} \cdot\left[\left(\nabla \Sigma, \Lambda^{2} \Theta\right)-\left(\nabla \Theta, \Lambda^{2} \Sigma\right)-\left(\varepsilon_{1}, \nabla \Lambda^{2} \Theta\right)+\left(\varepsilon_{2}, \nabla \Lambda^{2} \Sigma\right)\right] \\
& -\frac{\lambda_{s}}{\lambda}\left[\left(\varepsilon_{1}, \Lambda^{3} \Theta\right)-\left(\varepsilon_{2}, \Lambda^{3} \Sigma\right)\right]+2\left(\frac{\lambda_{s}}{\lambda}+b\right)\left(\Lambda \Sigma, \Lambda^{2} \Theta\right) \\
& -\left(R_{2}(\varepsilon), \Lambda^{2} \Theta\right)-\left(R_{1}(\varepsilon), \Lambda^{2} \Sigma\right)+\left(\operatorname{Im}\left(\Psi_{b}\right), \Lambda^{2} \Theta\right)+\left(\operatorname{Re}\left(\Psi_{b}\right), \Lambda^{2} \Sigma\right) .
\end{aligned}
$$

Proof. Take the inner product of (3.3) with $-\Lambda \Theta$ and of (3.4) with $\Lambda \Sigma$, sum the obtained equalities, use the orthogonality condition (1.56) and integrate by parts to get (3.12).

(3.13) follows by summing the inner product of (3.3) with $|y|^{2} \Sigma$ and of (3.4) with $|y|^{2} \Theta$ and the orthogonality condition (1.54).

Similarly, (3.14) follows by summing the inner product of (3.3) with $y \Sigma$ and of (3.4) with $y \Theta$ and the orthogonality condition (1.55).

Finally, (3.15) follows by summing the inner product of (3.3) with $\Lambda^{2} \Theta$ and of (3.4) with $-\Lambda^{2} \Sigma$ and the orthogonality condition (1.57).

Note that

$$
\begin{aligned}
\Lambda(f g) & =g \Lambda f+f \Lambda g-\frac{d}{2} f g \\
& =g \Lambda f+f y \cdot \nabla g \\
\Delta(\Lambda f) & =2 \Delta f+\Lambda(\Delta f),
\end{aligned}
$$

and using the orthogonality condition (1.57), we obtain the following lemma.

Lemma 3.4. There holds

$$
\begin{aligned}
& -\left(M_{+}(\varepsilon), \Lambda \Sigma\right)-\left(M_{-}(\varepsilon), \Lambda \Theta\right) \\
= & 2\left(\varepsilon_{1}, \Sigma+b \Lambda \Theta-\operatorname{Re}\left(\Psi_{b}\right)\right)+2\left(\varepsilon_{2}, \Theta-b \Lambda \Theta-\operatorname{Im}\left(\Psi_{b}\right)\right) \\
& -\left(\varepsilon_{1}, \operatorname{Re}\left(\Lambda \Psi_{b}\right)\right)-\left(\varepsilon_{2}, \operatorname{Im}\left(\Lambda \Psi_{b}\right)\right) .
\end{aligned}
$$


Thus, this together with (3.12) and (2.2) yields that

$$
\begin{aligned}
& b_{s}\left[\left(\partial_{b} \Theta, \Lambda \Sigma\right)-\left(\partial_{b} \Sigma, \Lambda \Theta\right)+\left(\epsilon_{1}, \partial_{b} \Lambda \Theta\right)-\left(\epsilon_{2}, \partial_{b} \Lambda \Sigma\right)\right] \\
= & 2 \Xi(s)+H\left(I_{N \lambda} \varepsilon, I_{N \lambda} \varepsilon\right)-\left(\varepsilon_{1}, \operatorname{Re}\left(\Lambda \Psi_{b}\right)\right)-\left(\varepsilon_{2}, \operatorname{Im}\left(\Lambda \Psi_{b}\right)\right) \\
& -\tilde{\gamma}_{s}\left[\left(\varepsilon_{1}, \Lambda \Sigma\right)+\left(\varepsilon_{2}, \Lambda \Theta\right)\right]-\frac{x_{s}}{\lambda} \cdot\left[\left(\varepsilon_{2}, \nabla \Lambda \Sigma\right)-\left(\varepsilon_{1}, \nabla \Lambda \Theta\right)\right] \\
& +O\left(\delta_{0}\left(\int\left|I_{N(s) \lambda(s)} \nabla \varepsilon(s)\right|^{2}+\int|\varepsilon(s)|^{2} e^{-|y|}\right)\right)+O\left(\Gamma_{b(s)}^{1-C \eta}\right)+F(s),
\end{aligned}
$$

with

$$
\begin{aligned}
& F(s)=\left(R_{2}(\epsilon)-R_{2}\left(I_{N \lambda} \epsilon\right), \Lambda \Theta\right)+\left(R_{1}(\epsilon)-R_{1}\left(I_{N \lambda} \epsilon\right), \Lambda \Sigma\right) \\
& +\widetilde{H}_{b}\left(I_{N \lambda} \epsilon, I_{N \lambda} \epsilon\right)+\left(\widetilde{R}_{1}\left(I_{N \lambda} \epsilon\right), \Lambda \Sigma\right)+\left(\widetilde{R}_{2}\left(I_{N \lambda} \epsilon\right), \Lambda \Theta\right), \\
\widetilde{H}_{b}(\epsilon, \epsilon):= & \int\left|V_{1}(y)\right|\left(\epsilon_{1}\right)^{2}+\int V_{2}(y)\left(\epsilon_{2}\right)^{2}+\int V_{12}(y) \epsilon_{1} \epsilon_{2}, \\
V_{1}(y)= & \frac{2}{d}\left(1+\frac{4}{d}\right)\left[\frac{\left|Q_{b}\right|^{\frac{4}{d}}}{\left|Q_{b}\right|^{4}} \Sigma^{3} y \cdot \nabla \Sigma-Q^{\frac{4}{d}-1} y \nabla Q\right] \\
+ & \frac{\left|Q_{b}\right|^{\frac{4}{d}}}{\left|Q_{b}\right|^{4}} \Theta^{2}\left[\frac{6}{d} \Sigma \Lambda \Sigma-\left(\frac{4}{d}+2\right) \Sigma^{2}-\Theta^{2}\right] \\
+ & \frac{2}{d} \frac{\left|Q_{b}\right|^{\frac{4}{d}}}{\left|Q_{b}\right|^{4}} \Theta \Lambda \Theta\left[\Theta^{2}+\left(\frac{4}{d}-1\right) \Sigma^{2}\right], \\
V_{2}(y)= & \frac{2}{d}\left[\frac{\left|Q_{b}\right|^{\frac{4}{d}}}{\left|Q_{b}\right|^{4}} \Sigma^{3} y \cdot \nabla \Sigma-Q^{\frac{4}{d}-1} y \cdot \nabla Q\right] \\
+ & \frac{\left|Q_{b}\right|^{\frac{4}{d}}}{\left|Q_{b}\right|^{4}} \Theta^{2}\left[\frac{2}{d}\left(\frac{4}{d}-1\right) \Sigma \Lambda \Sigma-\left(\frac{4}{d}+2\right) \Sigma^{2}-\left(\frac{4}{d}+1\right) \Theta^{2}\right] \\
+ & \frac{2 \Theta \Lambda \Theta}{d} \frac{\left|Q_{b}\right|^{\frac{4}{d}}}{\left|Q_{b}\right|^{4}}\left[3 \Sigma^{2}+\left(\frac{4}{d}+1\right) \Theta^{2}\right], \\
V_{12}(y)= & \frac{4}{d} \frac{\left|Q_{b}\right|^{\frac{4}{d}}}{\left|Q_{b}\right|^{4}}\left[\Theta\left(\Theta^{2} \Lambda \Sigma+\left(\frac{4}{d}-1\right)\left(\Sigma^{2} \Lambda \Sigma+\Sigma \Theta \Lambda \Theta\right)-2 \Sigma\left|Q_{b}\right|^{2}\right)+\Sigma^{3} \Lambda \Theta\right] .
\end{aligned}
$$

Moreover, the remainder $F$ can be bounded by

$$
|F| \leq \delta\left(\alpha^{*}\right)\left(\int\left|\nabla I_{N \lambda} \epsilon\right|^{2}+\int|\epsilon|^{2} e^{-|y|}\right)+\Gamma_{b}^{1-C \eta}
$$

Remark 3.5. By the estimation of $F(s)$, we can absorb this term into $\Gamma_{b(s)}^{1-C \eta}$. This is different from the dimension two case in [5].

Proof. The algebraic identity can be obtained directly from the proof of Lemma 3.2. It is only a matter to estimate the remainder term $F(s)$. Denote by

$$
\begin{gathered}
F_{1}(s)=\left(R_{2}(\epsilon)-R_{2}\left(I_{N \lambda} \epsilon\right), \Lambda \Theta\right)+\left(R_{1}(\epsilon)-R_{1}\left(I_{N \lambda} \epsilon\right), \Lambda \Sigma\right), \\
F_{2}(s)=F(s)-F_{1}(s) .
\end{gathered}
$$

Denote by

$$
\left\|I_{N \lambda} \epsilon\right\|_{H_{e x p}^{1}}^{2}:=\int\left|I_{N \lambda} \epsilon\right|^{2}+\int\left|I_{N \lambda} \epsilon\right|^{2} e^{-|y|} .
$$


Estimate of $F_{2}$ : From [18,

$$
\left|\widetilde{H}_{b}\left(I_{N \lambda} \epsilon, I_{N \lambda} \epsilon\right)\right|+\left|\left(\widetilde{R}_{1}\left(I_{N \lambda} \epsilon\right), \Lambda \Sigma\right)\right|+\left|\left(\widetilde{R}_{2}\left(I_{N \lambda} \epsilon\right), \Lambda \Theta\right)\right| \leq \delta_{0}\left\|I_{N \lambda} \epsilon\right\|_{H_{e x p}^{1}}^{2} .
$$

Estimate of $F_{1}$ : Note that $F_{1}$ can be written in the following form

$$
\begin{aligned}
F_{1}(s) & =\int|\epsilon|^{2}\left(\operatorname{Id}-I_{N \lambda}\right) \phi_{1}+\int\left[\left|I_{N \lambda} \epsilon\right|^{2}-I_{N \lambda}\left(|\epsilon|^{2}\right)\right] \phi_{2} \\
& +O\left(\int\left(|\epsilon|^{3} \chi_{d=3}+|\epsilon|^{2+\frac{2}{d}} \chi_{d \geq 4}\right) \phi_{3}\right)
\end{aligned}
$$

where $\phi_{1}, \phi_{2}, \phi_{3}$ are Schwartz functions built on $Q$ which decay exponentially as $r \rightarrow \infty$. We estimate

$$
\left.\left|\int\right| \epsilon\right|^{2}\left(\mathrm{Id}-I_{N \lambda}\right) \phi_{1} \mid \leq\|\epsilon\|_{L^{2}}\left\|\left(\operatorname{Id}-I_{N \lambda} \phi\right)\right\|_{L^{\infty}} \lesssim \frac{1}{N \lambda} \lesssim \Gamma_{b}^{10},
$$

and

$$
\begin{aligned}
& \left|\int\left[\left|I_{N \lambda} \epsilon\right|^{2}-I_{N \lambda}\left(|\epsilon|^{2}\right)\right] \phi_{2}\right| \\
\lesssim & \left\|\left(I_{N \lambda} \epsilon-\epsilon\right)\left|\phi_{2}\right|^{1 / 2}\right\|_{L^{2}}\left\|\left(I_{N \lambda} \epsilon+\epsilon\right)\left|\phi_{2}\right|^{1 / 2}\right\|_{L^{2}}+\left\|\epsilon|\phi|^{1 / 2}\right\|_{L^{2}}^{2}\left\|\left(\operatorname{Id}-I_{N \lambda}\right) \phi_{2}\right\|_{L^{\infty}} \\
\lesssim & \frac{1}{N \lambda} \lesssim \Gamma_{b}^{10} .
\end{aligned}
$$

To estimate the third term in $F_{1}$, we only deal with the case $d \geq 4$ here. Denote by $J_{N \lambda}=\mathrm{Id}-I_{N \lambda}$, and from the assumption $s>\frac{1}{1+\frac{4}{d}}=\frac{d}{d+4}$, we know that $\frac{2 d}{d-2 s}>2+\frac{2}{d}$, we use Sobolev embedding to estimate

$$
\begin{aligned}
& \left.\left.\left|\int\right| \epsilon\right|^{2+\frac{2}{d}} \phi_{3}\left|\leq C \int\right| I_{N \lambda} \epsilon\right|^{2+\frac{2}{d}}\left|\phi_{3}\right|+C \int\left|J_{N \lambda} \epsilon\right|^{2+\frac{2}{d}}\left|\phi_{3}\right|, \\
& \left\|I_{N \lambda} \epsilon\right\|_{L^{2+\frac{2}{d}\left(\left|\phi_{3}\right| d y\right)}} \leq\left\|I_{N \lambda} \epsilon\right\|_{L^{2}\left(\left|\phi_{3}\right| d y\right)}^{\theta_{1}}\left\|I_{N \lambda} \epsilon\right\|_{L^{\frac{2 d}{d-2}}\left(\left|\phi_{3}\right| d y\right)}^{1-\theta_{1}} \\
& \leq C\left\|I_{N \lambda} \epsilon\right\|_{L^{2}\left(\left|\phi_{3}\right| d y\right)}^{\theta_{1}}\left\|\nabla I_{N \lambda} \epsilon\right\|_{L^{2}}^{1-\theta_{1}} \leq C\left\|I_{N \lambda} \epsilon\right\|_{H_{e x p}^{1}},
\end{aligned}
$$

and

$$
\begin{aligned}
&\left\|J_{N \lambda} \epsilon\right\|_{L^{2+\frac{2}{d}\left(\left|\phi_{3}\right| d y\right)}} \leq\left\|J_{N \lambda} \epsilon\right\|_{L^{2}\left(\left|\phi_{3}\right| d y\right)}^{\theta_{2}}\left\|J_{N \lambda} \epsilon\right\|_{L^{\frac{2 d}{d-2 s}}\left(\left|\phi_{3}\right| d y\right)}^{1-\theta_{2}} \\
& \leq\left\|J_{N \lambda} \epsilon\right\|_{L^{2}}^{\theta_{2}}\left\|J_{N \lambda} \epsilon\right\|_{\dot{H}^{s}}^{1-\theta_{2}} \\
& \leq \frac{1}{(N \lambda)^{s \theta_{2}}}\left\|J_{N \lambda} \epsilon\right\|_{\dot{H}^{s}} \\
& \leq \frac{1}{(N \lambda)^{s \theta_{2}}}\left\|P_{\geq N} \epsilon\right\|_{\dot{H}^{s}} \\
& \leq \frac{1}{(N \lambda)^{s \theta_{2}}}\left\|\nabla I_{N \lambda} \epsilon\right\|_{L^{2}} \leq C\left\|I_{N \lambda} \epsilon\right\|_{H_{e x p}^{1}} .
\end{aligned}
$$

This completes the proof of Lemma3.4.

View the four equalities (3.13), (3.14), (3.15), (3.17) as a linear system, which is invertible, we obtain 
Lemma 3.6 (Control of the geometrical parameters). There holds:

$$
\begin{aligned}
\left|\frac{\lambda_{s}}{\lambda}+b\right|+\left|b_{s}\right| \leq & C\left(\Xi(s)+\int\left|\nabla I_{N(s) \lambda(s)} \varepsilon(s)\right|^{2}+\int|\varepsilon(s)|^{2} e^{-|y|}\right) \\
& +\Gamma_{b(s)}^{1-C \eta} \\
\left|\tilde{\gamma}_{s}-\frac{\left(\varepsilon_{1}, L_{+} \Lambda^{2} Q\right)}{\|\Lambda Q\|_{L^{2}}^{2}}\right|+\left|\frac{x_{s}}{\lambda}\right| \leq & \delta_{0}\left(\int|\varepsilon(s)|^{2} e^{-|y|}\right)^{\frac{1}{2}}+\Gamma_{b(s)}^{1-C \eta} \\
& +C\left(\Xi(s)+\int\left|\nabla I_{N(s) \lambda(s)} \varepsilon(s)\right|^{2}\right) .
\end{aligned}
$$

3.2. Virial dispersion. In this subsection, we will derive two global virial dispersive estimates at the heart of the control of the log-log regime in [18, 20]. We begin with the global virial estimate first established in [17, 18.

Lemma 3.7 (Global virial estimate, [5]). We have

$$
b_{s} \geq c_{0}\left(\Xi(s)+\int\left|\nabla I_{N(s) \lambda(s)} \varepsilon(s)\right|^{2}+\int|\varepsilon(s)|^{2} e^{-|y|}\right)-\Gamma_{b(s)}^{1-C \eta} .
$$

Next, we consider another dispersive control of a slightly different kind exhibited in [19, 20. The main idea is that the profile $Q_{b}+\zeta_{b}$ should be a better approximation of the solution. Let us introduce a cut off parameter

$$
A(t)=e^{\frac{2 a}{b(t)}} \quad \text { so that } \quad \Gamma_{b}^{-\frac{a}{2}} \leq A \leq \Gamma_{b}^{-\frac{3 a}{2}}
$$

for some small parameter $0<a \ll 1$ and

$$
\tilde{\zeta}=\chi\left(\frac{r}{A}\right) \zeta_{b}
$$

where $\chi(r)$ is a smooth cut-off function with

$$
\chi(r)=\left\{\begin{array}{lll}
1 & \text { if } & 0 \leq r \leq \frac{3}{2} \\
0 & \text { if } & r \geq 2 .
\end{array}\right.
$$

We remark that $\tilde{\zeta}$ is a small Schwartz function due to the $A$ localization. We next consider the new variable

$$
\tilde{\varepsilon}=\varepsilon-\tilde{\zeta}
$$

Then, by the same argument as Lemma 6 in [20] and the above, we obtain

Lemma 3.8 (Virial dispersion in the radiative regime). There holds for some universal constants $c_{1}>0$ and $s \in\left[s_{0}, s^{+}\right]$:

$$
\begin{aligned}
\left\{f_{1}(s)\right\}_{s} \geq & c_{1}\left(\Xi(s)+\int\left|\nabla I_{N(s) \lambda(s)} \tilde{\varepsilon}(s)\right|^{2}+\int|\widetilde{\varepsilon}(s)|^{2} e^{-|y|}+\Gamma_{b}\right) \\
& -\frac{1}{\delta_{1}} \int_{A}^{2 A}|\varepsilon|^{2},
\end{aligned}
$$

with

$$
f_{1}(s)=\frac{b}{4}\left\|y Q_{b}\right\|_{L^{2}}^{2}+\frac{1}{2} \operatorname{Im}\left(\int(y \cdot \nabla \tilde{\zeta}) \varepsilon \tilde{\zeta}\right)+\operatorname{Re}\left(\varepsilon_{2}, \Lambda \tilde{\zeta}\right)-\operatorname{Im}\left(\varepsilon_{1}, \Lambda \tilde{\zeta}\right)
$$


Proof. Step 1: Algebraic dispersive relation.

We have(See 20] for the calculation of principle terms)

$$
\begin{aligned}
\frac{d f_{1}}{d s} & =H\left(I_{N \lambda} \epsilon-\widetilde{\zeta}_{b}, I_{N \lambda} \epsilon-\widetilde{\zeta}_{b}\right)+\left(\epsilon_{1}-\operatorname{Re} \widetilde{\zeta}_{b}, \operatorname{Re} \Lambda F\right)+\left(\epsilon_{2}-\operatorname{Im} \widetilde{\zeta}_{b}, \operatorname{Im} \Lambda F\right) \\
& -2\left(\lambda^{2} E\left(I_{N} u\right)+\Xi(s)\right)+b_{s}\left[\left(\epsilon_{2}-\operatorname{Im} \widetilde{\zeta}, \partial_{b} \Lambda(\Sigma+\operatorname{Re} \widetilde{\zeta})\right)-\left(\epsilon_{1}-\operatorname{Re} \widetilde{\zeta}, \partial_{b} \Lambda(\Theta+\operatorname{Im} \widetilde{\zeta})\right)\right] \\
& -\frac{A_{s}}{A^{2}}\left[\left(\epsilon_{2}-\operatorname{Im} \widetilde{\zeta}, \Lambda\left(y \cdot(\nabla \chi)\left(\frac{y}{A}\right) \operatorname{Re} \widetilde{\zeta}\right)\right)-\left(\epsilon_{1}-\operatorname{Re} \widetilde{\zeta}, \Lambda\left(y \cdot(\nabla \chi)\left(\frac{y}{A}\right) \operatorname{Im} \widetilde{\zeta}\right)\right)\right] \\
& -\left(\frac{\lambda_{s}}{\lambda}+b\right)\left[\left(\epsilon_{2}-\operatorname{Im} \widetilde{\zeta}, \Lambda^{2}(\Sigma+\operatorname{Re} \widetilde{\zeta})\right)-\left(\epsilon_{1}-\operatorname{Re} \widetilde{\zeta}, \Lambda^{2}(\Theta+\operatorname{Im} \widetilde{\zeta})\right)\right] \\
& -\widetilde{\gamma}_{s}\left[\left(\epsilon_{1}-\operatorname{Re} \widetilde{\zeta}, \Lambda(\Sigma+\operatorname{Re} \widetilde{\zeta})\right)+\left(\epsilon_{2}-\operatorname{Im} \widetilde{\zeta}, \Lambda(\Theta+\operatorname{Im} \widetilde{\zeta})\right)\right] \\
& -\frac{x_{s}}{\lambda} \cdot\left[\left(\epsilon_{2}-\operatorname{Im} \widetilde{\zeta}, \nabla \Lambda(\Sigma+\operatorname{Re} \widetilde{\zeta})\right)-\left(\epsilon_{1}-\operatorname{Re} \widetilde{\zeta}, \nabla \Lambda(\Theta+\operatorname{Im} \widetilde{\zeta})\right)\right] \\
& +\left(R_{1}(\epsilon), \Lambda \operatorname{Re} \widetilde{\zeta}\right)+\left(R_{2}(\epsilon), \Lambda \operatorname{Im} \widetilde{\zeta}\right) \\
& +\left(\epsilon_{1}-\operatorname{Re} \widetilde{\zeta}_{b}, \Lambda\left(\left(1+\frac{4}{d}\right) Q^{\frac{4}{d}} \operatorname{Im} \widetilde{\zeta_{b}}\right)\right)+\left(\epsilon_{2}-\operatorname{Im} \widetilde{\zeta}_{b}, \Lambda\left(Q^{\frac{4}{d}} \operatorname{Im} \widetilde{\zeta}_{b}\right)\right) \\
& +\left(\epsilon_{1}, \widetilde{L}\right)+\left(\epsilon_{2}, \widetilde{K}\right)+\Upsilon_{N \lambda}+2 \Xi(s)+\mathcal{R e m a i n d e r}
\end{aligned}
$$

with

$$
\begin{aligned}
& \widetilde{L}=\left[\left(\frac{4 \Sigma^{2}}{d\left|Q_{b}\right|^{2}}+1\right)\left|Q_{b}\right|^{\frac{4}{d}}-\left(1+\frac{4}{d}\right) Q^{\frac{4}{d}}\right] \Lambda \operatorname{Re} \widetilde{\zeta}_{b}+\frac{4 \Sigma \Theta}{d\left|Q_{b}\right|^{2}}\left|Q_{b}\right|^{\frac{4}{d}} \Lambda \operatorname{Im} \widetilde{\zeta}_{b}, \\
& \widetilde{K}=\left[\left(\frac{4 \Theta^{2}}{d\left|Q_{b}\right|^{2}}+1\right)\left|Q_{b}\right|^{\frac{4}{d}}-Q^{\frac{4}{d}}\right] \Lambda \operatorname{Im} \widetilde{\zeta}_{b}+\frac{4 \Sigma \Theta}{d\left|Q_{b}\right|^{2}}\left|Q_{b}\right|^{\frac{4}{d}} \Lambda \operatorname{Re} \widetilde{\zeta}_{b},
\end{aligned}
$$

coming from the error term:

$$
\begin{gathered}
\left(L_{+} \epsilon_{1}+b \Lambda \epsilon_{2}, \Lambda \operatorname{Re} \widetilde{\zeta}_{b}\right)+\left(L_{-} \epsilon_{2}-b \Lambda \epsilon_{1}, \Lambda \operatorname{Im} \widetilde{\zeta}_{b}\right) \\
-\left(M_{+}(\epsilon)+b \Lambda \epsilon_{2}, \Lambda \operatorname{Re} \widetilde{\zeta}_{b}\right)-\left(M_{-}(\epsilon)-b \Lambda \epsilon_{1}, \Lambda \operatorname{Im} \widetilde{\zeta}_{b}\right) .
\end{gathered}
$$

Another error terms

$$
\begin{aligned}
\Upsilon_{N \lambda} & =\left(\left(I_{N \lambda}-I d\right) \epsilon_{1}, \operatorname{Re} \Lambda \Psi_{b}\right)+\left(\left(I_{N \lambda}-I d\right) \epsilon_{2}, \operatorname{Im} \Lambda \Psi_{b}\right) \\
& +\left(L_{+}\left(I_{N \lambda}-I d\right) \epsilon_{1}+b \Lambda\left(I_{N \lambda}-I d\right) \epsilon_{2}, \Lambda \operatorname{Re} \widetilde{\zeta}_{b}\right) \\
& +\left(L_{-}\left(I_{N \lambda}-I d\right) \epsilon_{2}-b \Lambda\left(I_{N \lambda}-I d\right) \epsilon_{1}, \Lambda \operatorname{Im} \widetilde{\zeta}_{b}\right) \\
& +\left(\left(I_{N \lambda}-I d\right) \epsilon_{1}, \Lambda\left(\operatorname{Re} F+\left(1+\frac{4}{d}\right) Q^{\frac{4}{d}} \operatorname{Re} \widetilde{\zeta}_{b}\right)\right) \\
& +\left(\left(I_{N \lambda}-I d\right) \epsilon_{2}, \Lambda\left(\operatorname{Im} F+Q^{\frac{4}{d}} \operatorname{Im} \widetilde{\zeta}_{b}\right)\right)
\end{aligned}
$$

and

$$
\begin{aligned}
\text { Reminder } & =\widetilde{H}_{b}\left(I_{N \lambda} \epsilon, I_{N \lambda} \epsilon\right)+\left(R_{1}(\epsilon)-R_{1}\left(I_{N \lambda} \epsilon\right), \Lambda \Sigma\right)+\left(R_{2}(\epsilon)-R_{2}\left(I_{N \lambda} \epsilon\right), \Lambda \Theta\right) \\
& +\left(\widetilde{R}_{1}\left(I_{N \lambda} \epsilon\right), \Lambda \Sigma\right)+\left(\widetilde{R}_{2}\left(I_{N \lambda} \epsilon\right), \Lambda \Theta\right)-\frac{d}{d+2} \int J\left(I_{N \lambda} \epsilon\right) \\
& +2 E\left(I_{N \lambda}\left(Q_{b}+\epsilon\right)\right)-2 E\left(Q_{b}+I_{N \lambda} \epsilon\right)-2 \operatorname{Re} \int\left(I_{N \lambda}-\operatorname{Id}\right)\left(Q_{b}-i b \Lambda Q_{b}-\Psi_{b}\right) \bar{\epsilon}
\end{aligned}
$$


Step 2: Control of interaction terms.

$\underline{\text { Claim 1: }}$

$$
\begin{aligned}
& \int\left|\left(\operatorname{Id}-I_{N \lambda}\right) \epsilon\right|^{2} e^{-|y|}=O\left(\Gamma_{b}^{1+z_{0}}\right), \\
& \int\left|\nabla I_{N \lambda} \widetilde{\epsilon}\right|^{2}+\int|\widetilde{\epsilon}|^{2} e^{-|y|}=\int\left|\nabla\left(I_{N \lambda} \epsilon-\widetilde{\zeta}_{b}\right)\right|^{2}+\int\left|I_{N \lambda} \epsilon-\widetilde{\zeta}_{b}\right|^{2} e^{-|y|}+O\left(\Gamma_{b}^{1+z_{0}}\right), \\
& \int|\epsilon|^{2} e^{-|y|} \leq 2 \int|\widetilde{\epsilon}|^{2} e^{-|y|}+\Gamma_{b}^{1+z_{0}} \\
& \int\left|\nabla I_{N \lambda} \epsilon\right|^{2} \leq C \int\left|\nabla I_{N \lambda} \widetilde{\epsilon}\right|^{2}+\Gamma_{b}^{1-C \eta}
\end{aligned}
$$

Indeed, the first estimate comes from the boundness of $I_{N \lambda} \epsilon$ in $H^{1}$ ( thus the boundness of $\epsilon$ in $H^{s}$ ) as well as $(N \lambda)^{-s}=O\left(\Gamma_{b}^{1+z_{0}}\right)$ by our bootstrap assumption. Similarly, the second estimate comes from the smallness of $\widetilde{\zeta}_{b}$ :

$$
\left\|\widetilde{\zeta}_{b}\right\|_{H^{1}} \leq \Gamma_{b}^{1-C \eta}
$$

and the error estimate $\left\|\operatorname{Id}-I_{N \lambda}\right\|_{H^{1} \rightarrow L^{2}} \leq \frac{1}{N \lambda}$. The third inequality comes from the property of $\zeta$ :

$$
\begin{aligned}
&\left\|\zeta_{b}(y) e^{-\frac{\sigma \theta(b|y|)}{b}}\right\|_{L^{\infty}\left(|y| \leq R_{b}\right)} \leq \Gamma_{b}^{\frac{1}{2}+\frac{\sigma}{10}}, \forall \sigma \in(0,5), \quad\left\|\zeta_{b}|y|^{d / 2}\right\|_{L^{\infty}\left(|y|>R_{b}\right)} \leq \Gamma_{b}^{\frac{1}{2}-C \eta} . \\
& \int|\epsilon|^{2} e^{-|y|} \leq 2 \int|\widetilde{\epsilon}|^{2} e^{-|y|}+2 \int|\widetilde{\zeta}|^{2} e^{-|y|} \\
& \leq 2 \int|\widetilde{\epsilon}|^{2} e^{-|y|}+2 \int \chi_{A}(y)^{2}\left|\zeta_{b}\right|^{2} e^{-\frac{\theta(b|y|)}{|b|}} \\
& \leq 2 \int|\widetilde{\epsilon}|^{2} e^{-|y|}+2 \Gamma_{b}^{1+\frac{1-C \eta}{5}} R_{b}+2 \Gamma_{b}^{1-2 C \eta} \log (2 A) e^{-\frac{\theta(2 \sqrt{1-\eta})}{|b|}} \\
& \leq 2 \int|\widetilde{\epsilon}|^{2} e^{-|y|}+\Gamma_{b}^{1+z_{0}} .
\end{aligned}
$$

Claim 2:

(1) For $d \geq 3$ and any $B \geq 2$,

$$
\int_{|y| \leq B}\left|\widetilde{\epsilon}^{2}\right| \leq C B^{2}\left(\int\left|\nabla I_{N \lambda} \widetilde{\epsilon}\right|^{2}+\int|\widetilde{\epsilon}|^{2} e^{-|y|}\right)+\Gamma_{b}^{1+z_{0}}
$$

(2) Second order interaction : for $R(\epsilon)=R_{1}(\epsilon)$ or $R_{2}(\epsilon)$,

$$
\int|R(\epsilon)| e^{-(1-C \eta) \frac{\theta(b|y|)}{|b|}} \leq C\left(\int\left|\nabla I_{N \lambda} \widetilde{\epsilon}\right|^{2}+\int|\widetilde{\epsilon}|^{2} e^{-|y|}\right)+\Gamma_{b}^{1+z_{0}} .
$$

(3) Small second-order interaction:

$$
\int|R(\epsilon)|\left(\left|\widetilde{\zeta}_{b}\right|+\left|y \cdot \nabla \widetilde{\zeta}_{b}\right|\right) \leq \delta\left(\alpha^{*}\right)\left(\int\left|\nabla I_{N \lambda} \widetilde{\epsilon}\right|^{2}+\int|\widetilde{\epsilon}|^{2} e^{-|y|}\right)+\Gamma_{b}^{1+z_{0}}
$$

(4) Small second-order scalar products: For any polynomial $P(y)$ and integers $0 \leq k \leq 2,0 \leq l \leq 1$, there exists $C>0$ such that

$$
\int|\widetilde{\epsilon}| P(y) \mid\left(\left|\nabla_{y}^{k} \widetilde{\zeta}_{b}\right|+\left|\nabla_{y}^{l} \partial_{b} \widetilde{\zeta}_{b}\right|\right) \leq \Gamma_{b}^{C}\left(\int\left|\nabla I_{N \lambda} \widetilde{\epsilon}\right|^{2}+\int|\widetilde{\epsilon}|^{2} e^{-|y|}\right)^{\frac{1}{2}}+\Gamma_{b}^{\frac{1}{2}+z_{0}}
$$


(5) Cut-off $\chi_{A}$ induced estimates

$$
\int|\epsilon|(|F|+|y \cdot F|) \leq C \Gamma_{b}^{\frac{1}{2}}\left(\int_{A \leq|y| \leq 2 A}|\epsilon|^{2}\right)^{\frac{1}{2}} .
$$

Proof:

(1) follows from $\|\widetilde{\epsilon}\|_{L^{2}(|y| \leq B)} \leq\left\|I_{N \lambda} \widetilde{\epsilon}\right\|_{L^{2}(|y| \leq B)}+\left\|\left(\operatorname{Id}-I_{N \lambda}\right) \widetilde{\epsilon}\right\|_{L^{2}}$ and classical inequality(see [20])

$$
\int_{|y| \leq B}|v|^{2} \leq C B^{2}\left(\int|\nabla v|^{2}+\int|v|^{2} e^{-|y|}\right)
$$

combining with the first inequality in claim 1 .

For (2), from the classical inequality (see [20])

$$
\begin{gathered}
|R(\epsilon)| \leq C|\epsilon|^{2} e^{-\left(\frac{4}{d}-1\right)(1-C \eta) \frac{\theta(b|y|)}{|b|}}+|\epsilon|^{1+\frac{4}{d}}, \quad d \leq 3, \\
|R(\epsilon)| \leq C \min \left(|\epsilon|^{2} e^{-\left(\frac{4}{d}-1\right)(1-C \eta) \frac{\theta(b|y|)}{|b|}},|\epsilon|^{1+\frac{4}{d}}\right), \quad d \geq 4,
\end{gathered}
$$

Using the classical inequality

$$
\int|v|^{2} e^{-\kappa|y|} \leq C\left(\int|\nabla v|^{2}+\int|v|^{2} e^{-|y|}\right)
$$

and the third inequality in claim 1 , for $d \geq 4$, we have

$$
\int|\epsilon|^{2} e^{-\left(\frac{4}{d}-1\right)(1-C \eta) \frac{\theta(b|y|)}{|b|}} \leq \int|\widetilde{\epsilon}|^{2} e^{-\frac{4}{d}(1-C \eta) \frac{\theta(b|y|)}{|b|}}+\Gamma_{b}^{1+z_{0}}
$$

we conclude by replacing $\tilde{\epsilon}$ to $I_{N \lambda} \tilde{\epsilon}$ and an error term can be absorbed into $\Gamma_{b}^{1+z_{0}}$. For $d=3$, we easily have

$$
\int|\epsilon|^{\frac{7}{7}} e^{-(1-C \eta) \frac{\theta(b|y|)}{|b|}} \leq C \int|\widetilde{\epsilon}|^{\frac{7}{3}} e^{-(1-C \eta) \frac{\theta(b|y|)}{|b|}}+\Gamma_{b}^{1+z_{0}}
$$

since $\frac{7}{3}>2$.

For (4), we do the case $d \geq 4$ here. We estimate it by

$$
\begin{aligned}
& \int|\epsilon|^{1+\frac{4}{d}}\left(\left|\widetilde{\zeta}_{b}\right|+\left|y \cdot \nabla \widetilde{\zeta}_{b}\right|\right) \\
\leq & \Gamma_{b}^{\frac{1}{2}(1-C \eta)} \int_{|y| \leq 2 A}|\epsilon|^{1+\frac{4}{d}} \\
\leq & C \Gamma_{b}^{\frac{1}{2}(1-C \eta)} \int_{|y| \leq 2 A}|\widetilde{\epsilon}|^{1+\frac{4}{d}}+\Gamma_{b}^{1+z_{0}} \\
\leq & C \Gamma_{b}^{\frac{1}{2}(1-C \eta)} \int_{|y| \leq 2 A}\left(\left|I_{N \lambda} \widetilde{\epsilon}^{1+\frac{4}{d}}+\right|\left(\mathrm{Id}-I_{N \lambda}\right) \widetilde{\epsilon}^{1+\frac{4}{d}}\right)+\Gamma_{b}^{1+z_{0}} \\
\leq & C \Gamma_{b}^{\frac{1}{2}(1-C \eta)} A^{C}\left(\int_{|y| \leq 2 A}\left|I_{N \lambda} \widetilde{\epsilon}\right|^{\frac{2 d}{d-2}}\right)^{\frac{(d+4)(d-2)}{2 d^{2}}}+\Gamma_{b}^{\frac{1}{2}(1-C \eta)} A^{\frac{2 d}{1-\frac{4}{d}}} \|\left.\left(\mathrm{Id}-I_{N \lambda}\right) \widetilde{\epsilon}\right|_{L^{\frac{1+2}{2}}}+\Gamma_{b}^{1+z_{0}} \\
\leq & C \Gamma_{b}^{\frac{1}{2}(1-C \eta)} A^{C} \|\left.\nabla I_{N \lambda} \widetilde{\epsilon}\right|_{L^{2}} ^{\frac{d+4}{d}}+\Gamma_{b}^{1+z_{0}} \\
\leq & \Gamma_{b}^{C}\left\|\nabla I_{N \lambda} \widetilde{\epsilon}\right\|_{L^{2}}^{2}+\Gamma_{b}^{1+z_{0}},
\end{aligned}
$$

provided that $a>0, \eta>0$ are small enough $\left(A=e^{\frac{2 a \theta(2)}{b}}\right)$. 
(4) follows from the classical pointwise bound

$$
\left|P(y)\left(\left|\nabla_{y}^{k} \widetilde{\zeta}_{b}\right|+\left|\nabla_{y}^{l} \partial_{b} \widetilde{\zeta}_{b}\right|\right)\right| \leq A^{C} \Gamma_{b}^{\frac{1}{2}-C \eta}
$$

and (1).

(5) follows from the pointwise bound

$$
\|F\|_{L^{\infty}}+\|y \cdot \nabla F\|_{L^{\infty}} \leq \frac{C \Gamma_{b}^{\frac{1}{2}}}{A^{\frac{d}{2}}}
$$

and Cauchy-Schwartz inequality.

Step 3: Estimate of terms involving geometric parameters:

Denote by $\widetilde{\epsilon}_{1}=\epsilon_{1}-\operatorname{Re} \widetilde{\zeta}_{b}, \widetilde{\epsilon}_{2}=\epsilon_{2}-\operatorname{Re} \widetilde{\zeta}_{2}$, the terms to be estimated are:

$$
\begin{aligned}
\operatorname{Term}_{1} & =b_{s}\left[\left(\widetilde{\epsilon}_{2}, \partial_{b} \Lambda\left(\Sigma+\operatorname{Re} \widetilde{\zeta}_{b}\right)\right)-\left(\widetilde{\epsilon}_{1}, \partial_{b} \Lambda\left(\Theta+\operatorname{Im} \widetilde{\zeta}_{b}\right)\right)\right], \\
\operatorname{Term}_{2} & =\left(\frac{\lambda_{s}}{\lambda}+b\right)\left[\left(\widetilde{\epsilon}_{2}, \Lambda^{2}\left(\Sigma+\operatorname{Re} \widetilde{\zeta}_{b}\right)\right)-\left(\widetilde{\epsilon}_{1}, \Lambda^{2}\left(\Theta+\operatorname{Im} \widetilde{\zeta}_{b}\right)\right)\right], \\
\operatorname{Term}_{3} & =\frac{x_{s}}{\lambda} \cdot\left[\left(\widetilde{\epsilon}_{2}, \nabla \Lambda\left(\Sigma+\operatorname{Re} \widetilde{\zeta}_{b}\right)\right)-\left(\widetilde{\epsilon}_{1}, \nabla \Lambda\left(\Theta+\operatorname{Im} \widetilde{\zeta}_{b}\right)\right)\right], \\
\operatorname{Term}_{4} & =\widetilde{\gamma}_{s}\left[\left(\widetilde{\epsilon}_{1}, \Lambda\left(\Sigma+\operatorname{Re} \widetilde{\zeta}_{b}\right)\right)+\left(\widetilde{\epsilon}_{2}, \Lambda\left(\Theta+\operatorname{Im} \widetilde{\zeta}_{b}\right)\right)\right], \\
\operatorname{Term}_{5} & =\frac{A_{s}}{A}\left[\left(\widetilde{\epsilon}_{2}, \Lambda\left(y \cdot \nabla \chi\left(\frac{y}{A}\right) \operatorname{Re} \widetilde{\zeta}_{b}\right)\right)-\left(\widetilde{\epsilon}_{1}, \Lambda\left(y \cdot \nabla \chi\left(\frac{y}{A}\right) \operatorname{Im} \widetilde{\zeta}_{b}\right)\right)\right]
\end{aligned}
$$

Claim 3:

$\left|\operatorname{Term}_{1}\right|+\left|\operatorname{Term}_{2}\right|+\left|\operatorname{Term}_{3}\right| \leq \delta\left(\alpha^{*}\right)\left(\Xi(s)+\int\left|I_{N \lambda} \widetilde{\epsilon}\right|^{2}+\int|\widetilde{\epsilon}|^{2} e^{-|y|}\right)+\Gamma_{b}^{1+z_{0}}$.

$\left|\operatorname{Term}_{4}-\frac{\left(\widetilde{\epsilon}_{1}, L_{+} \Lambda^{2} Q\right)\left(\widetilde{\epsilon}_{1}, \Lambda Q\right)}{\|\Lambda Q\|_{L^{2}}^{2}}\right| \leq \delta\left(\alpha^{*}\right)\left(\Xi(s)+\left.\int\left|I_{N \lambda} \widetilde{\epsilon}^{2}+\int\right| \widetilde{\epsilon}\right|^{2} e^{-|y|}\right)+\Gamma_{b}^{1+z_{0}}$.

$$
\left|\operatorname{Term}_{5}\right| \leq \delta\left(\alpha^{*}\right)\left(\int\left|I_{N \lambda} \widetilde{\epsilon}\right|^{2}+\int|\widetilde{\epsilon}|^{2} e^{-|y|}\right)+\Gamma_{b}^{1+z_{0}}
$$

Proof:

For (1), the three terms on the left hand side can be estimated in a similar way by using Lemma 3.6 and Claim 1.

$$
\begin{aligned}
\mid \text { Term }_{1} \mid & \leq\left|b_{s}\right|\left(\int|\widetilde{\epsilon}|^{2} e^{-\kappa|y|}\right)^{\frac{1}{2}} \\
& \leq C\left(\Xi(s)+\int\left|\nabla I_{N \lambda} \epsilon\right|^{2}+\int|\epsilon|^{2} e^{-|y|}+\Gamma_{b}^{1-C \eta}\right)\left(\int|\widetilde{\epsilon}|^{2} e^{-|y|}\right)^{\frac{1}{2}} \\
& \leq C\left(\Xi(s)+\int\left|\nabla I_{N \lambda} \widetilde{\epsilon}\right|^{2}+\int|\widetilde{\epsilon}|^{2} e^{-|y|}+\Gamma_{b}^{1-C \eta}\right)\left(\int|\widetilde{\epsilon}|^{2} e^{-|y|}\right)^{\frac{1}{2}} \\
& \leq \delta\left(\alpha^{*}\right)\left(\Xi(s)+\int\left|\nabla I_{N \lambda} \widetilde{\epsilon}\right|^{2}+\int|\widetilde{\epsilon}|^{2} e^{-|y|}\right)+\Gamma_{b}^{1+z_{0}}
\end{aligned}
$$


where in the last step, we write the term

$$
\Gamma_{b}^{1-C \eta}\left(\int|\widetilde{\epsilon}|^{2} e^{-|y|}\right)^{\frac{1}{2}}=\Gamma_{b}^{1-C \eta-\eta^{\prime}}\left(\Gamma_{b}^{\eta^{\prime}}\left(\int \mid \widetilde{\epsilon}^{2} e^{-|y|}\right)^{\frac{1}{2}}\right)
$$

and use the elementary inequality $X Y \leq \frac{X^{2}+Y^{2}}{2}$.

For (2), the left hand side can be estimated as

$$
\begin{aligned}
& \left|\widetilde{\gamma}_{s}-\frac{\left(\widetilde{\epsilon_{1}}, L_{+} \Lambda^{2} Q\right)}{\|\Lambda Q\|_{L^{2}}^{2}}\right|\left|\left(\widetilde{\epsilon_{1}}, \Lambda\left(\Sigma+\operatorname{Re} \widetilde{\zeta}_{b}\right)\right)+\left(\widetilde{\epsilon_{2}}, \Lambda\left(\Theta+\operatorname{Im} \widetilde{\zeta}_{b}\right)\right)\right| \\
+ & \left|\frac{\left(\widetilde{\epsilon_{1}}, L_{+} \Lambda^{2} Q\right)}{\|\Lambda Q\|_{L^{2}}^{2}}\right|\left|\left(\widetilde{\epsilon}_{1}, \Lambda\left(\Sigma-Q+\operatorname{Re} \widetilde{\zeta}_{b}\right)\right)+\left(\widetilde{\epsilon_{2}}, \Lambda\left(\Theta+\operatorname{Im} \widetilde{\zeta}_{b}\right)\right)\right| .
\end{aligned}
$$

From Lemma 3.6,

$$
\begin{aligned}
\left|\widetilde{\gamma}_{s}-\frac{\left(\widetilde{\epsilon_{1}}, L_{+} \Lambda^{2} Q\right)}{\|\Lambda Q\|_{L^{2}}^{2}}\right| & \leq\left|\widetilde{\gamma}_{s}-\frac{\left(\epsilon_{1}, L_{+} \Lambda^{2} Q\right)}{\|\Lambda Q\|_{L^{2}}^{2}}\right|+\frac{\left|\left(\operatorname{Re} \widetilde{\zeta}_{b}, L_{+}^{2} \Lambda^{2} Q\right)\right|}{\|\Lambda Q\|_{L^{2}}^{2}} \\
& \leq \delta\left(\alpha^{*}\right)\left(\int\left|\nabla I_{N \lambda} \widetilde{\epsilon}\right|^{2}+\int|\widetilde{\epsilon}|^{2} e^{-|y|}\right)^{\frac{1}{2}}+\Gamma_{b}^{\frac{1}{2}+z_{0}^{\prime}}+C \Xi(s)
\end{aligned}
$$

since

$$
\int\left|\widetilde{\zeta}_{b}\right|\left|L^{+} \Lambda^{2} Q\right| \leq C \int\left|\widetilde{\zeta}_{b}\right| e^{-\kappa|y|} \leq \Gamma_{b}^{\frac{1}{2}+z_{0}^{\prime}}
$$

thanks to the property of $\zeta_{b}$, (see 20). Now (2) follows from (4) of Claim 2.

For (3), we note that $\left|\frac{A_{s}}{A}\right| \leq C\left|\frac{b_{s}}{b^{2}}\right|$ and

$$
\left\|\Lambda\left(y \cdot \nabla\left(\frac{y}{A}\right) \zeta_{b}\right)\right\|_{L^{\infty}} \leq A^{C} \Gamma_{b}^{1-C \eta},
$$

thus

$$
\begin{aligned}
\mid \text { Term }_{5} \mid & \leq C\left|\frac{b_{s}}{b^{2}}\right| A^{C} \Gamma_{b}^{1-C \eta}\left(\int_{|y| \leq 2 A}|\widetilde{\epsilon}|^{2}\right)^{\frac{1}{2}} \\
& \leq C\left|\frac{b_{s}}{b^{2}}\right| A^{C} \Gamma_{b}^{1-C \eta}\left[A\left(\int\left|\nabla I_{N \lambda} \widetilde{\epsilon}\right|^{2}+\int \mid \widetilde{\epsilon}^{2} e^{-|y|}\right)^{\frac{1}{2}}+\Gamma_{b}^{\frac{1}{2}+z_{0}}\right] \\
& \leq \delta\left(\alpha^{*}\right)\left(\int\left|I_{N \lambda} \widetilde{\epsilon}\right|^{2}+\int \mid \widetilde{\epsilon}^{2} e^{-|y|}\right)+\Gamma_{b}^{1+z_{0}}
\end{aligned}
$$

by writing that

$$
\|\widetilde{\epsilon}\|_{L^{2}(|y| \leq 2 A)} \leq\left\|I_{N \lambda} \widetilde{\epsilon}\right\|_{L^{2}(|y| \leq 2 A)}+\left\|\left(\operatorname{Id}-I_{N \lambda}\right) \widetilde{\epsilon}\right\|_{L^{2}}
$$

and using (1) of Claim 1.

Step 4: Estimate of degenerate scalar products.

\section{Claim 4:}

(1)

$$
\left(\widetilde{\epsilon_{1}}, Q\right)^{2} \leq \delta\left(\alpha^{*}\right)\left(\int\left|\nabla I_{N \lambda} \widetilde{\epsilon}\right|^{2}+\int|\widetilde{\epsilon}|^{2} e^{-|y|}+\Xi(s)\right)+\Gamma_{b}^{1+z_{0}}
$$

$$
\left(\widetilde{\epsilon}_{2}\right)^{2} \leq \delta\left(\alpha^{*}\right)\left(\int\left|\nabla I_{N \lambda} \widetilde{\epsilon}^{2}+\int\right| \widetilde{\epsilon}^{2} e^{-|y|}\right)+\Gamma_{b}^{1+z_{0}} .
$$


(3)

$$
\begin{aligned}
& \left(\widetilde{\epsilon_{1}},|y|^{2} Q\right)^{2}+\left(\widetilde{\epsilon_{1}}, y Q\right)^{2}+\left(\widetilde{\epsilon_{2}}, \Lambda Q\right)^{2}+\left(\widetilde{\epsilon_{2}}, \Lambda^{2} Q\right)^{2} \\
\leq & \delta\left(\alpha^{*}\right)\left(\int\left|\nabla I_{N \lambda} \widetilde{\epsilon}\right|^{2}+\int|\widetilde{\epsilon}|^{2} e^{-|y|}\right)+\Gamma_{b}^{1+z_{0}}
\end{aligned}
$$

Proof:

We first indicate that $\Xi(s) \leq \delta\left(\delta^{*}\right)$. Essentially,

$$
\Xi(s)=\frac{\lambda^{2}(s)}{2} \int\left|J_{N(s)} G(0, x)\right|^{2} d x
$$

with $J_{N}=\mathrm{Id}-I_{N}$. Recall that

$$
G(0, x)=\frac{1}{\lambda(0)^{\frac{d}{2}}}\left(Q_{b()}+g(0)\right)\left(\frac{x-x(0)}{\lambda(0)}\right) e^{-i \gamma(0)}
$$

we estimate

$$
\begin{aligned}
\frac{\lambda^{2}(s)}{2}\left\|J_{N(s)} G(0)\right\|_{L^{2}}^{2} & \leq \frac{\lambda(s)^{2}}{\lambda(0)^{2}}\|\nabla g(0)\|_{L^{2}}^{2}+\left\|J_{N(s) \lambda(0)}\left(\nabla Q_{b(0)}\right)\right\|_{L^{2}}^{2} \\
& \leq C \Gamma_{b(0)}^{\frac{3}{4}}+\frac{1}{N(s) \lambda(s)} \frac{\lambda(s)}{\lambda(0)} \\
& \leq \delta\left(\alpha^{*}\right)
\end{aligned}
$$

thanks to the bootstrap assumption.

Now (1) follows from the following estimate induced by almost conservation of energy:

$$
\left(\widetilde{\epsilon}_{1}, Q\right)^{2} \leq \delta\left(\alpha^{*}\right)\left(\int\left|\nabla I_{N \lambda} \widetilde{\epsilon}\right|^{2}+\int|\widetilde{\epsilon}|^{2} e^{-|y|}+\Xi(s)\right)+\Gamma_{b}^{1+z_{0}}+\left(\lambda^{2} E\left(I_{N} u\right)\right)^{2}
$$

Indeed, we have already seen in Step 3 that

$$
\int\left|\widetilde{\zeta}_{b}\right|^{2} e^{-\kappa|y|} \leq \Gamma_{b}^{\frac{1}{2}+z_{0}}
$$

Thus we could replace the left hand side by $\left(\epsilon_{1}, Q\right)^{2}$. From the almost conservation of energy Lemma 3.2, we have

$$
\begin{aligned}
2\left(\epsilon_{1}, Q\right)= & -2\left(\epsilon_{1}, \Sigma-Q+b \Lambda \Theta\right)-2(\epsilon, \Theta-b \Lambda \Sigma)+2\left(\epsilon_{1}, \operatorname{Re} \Psi\right)+2\left(\epsilon_{2}, \operatorname{Im} \Psi\right) \\
& +2 \Xi(s)+O\left(\int\left|\nabla I_{N \lambda} \epsilon\right|^{2}+\int|\epsilon|^{2} e^{-|y|}+\Gamma_{b}^{1-C \eta}\right)
\end{aligned}
$$

Then we conclude by the estimates

$$
\begin{gathered}
\left|\operatorname{Re}\left(\epsilon, Q_{b}-Q+i b \Lambda Q_{b}\right)\right| \leq \delta\left(\alpha^{*}\right)\left(\int\left|\nabla I_{N \lambda} \epsilon\right|^{2}+\int|\epsilon|^{2} e^{-|y|}\right)^{\frac{1}{2}}+\Gamma_{b}^{\frac{1}{2}+z_{0}} \\
\left|\int\right| \epsilon|| \Psi||^{2} \leq \Gamma_{b}^{1-C \eta}\left(\int_{R_{b}^{-} \leq|y| \leq R_{b}}|\epsilon|\right)^{2} \\
\leq \Gamma_{b}^{1-C \eta}\left(\int\left|\nabla I_{N \lambda} \epsilon\right|^{2}+\int|\epsilon|^{2} e^{-|y|}\right)^{\frac{1}{2}}+\Gamma_{b}^{\frac{1}{2}+z_{0}}
\end{gathered}
$$

and the Claim 1. 
(2) follows from the almost conservation of momentum

$$
\begin{aligned}
-2\left(\varepsilon_{2}, \nabla Q\right)= & \lambda P\left(I_{N} u\right)-\left(P\left(I_{N \lambda}\left(Q_{b}+\varepsilon\right)\right)-P\left(Q_{b}+I_{N \lambda} \varepsilon\right)\right) \\
& -2\left(\varepsilon_{2}, \nabla(Q-\Sigma)\right)-2\left(\varepsilon_{2},\left(\mathrm{Id}-I_{N \lambda}\right) \nabla \Sigma\right) \\
& -2 \int I_{N \lambda} \varepsilon_{1} \nabla \Theta-2 \int I_{N \lambda} \varepsilon_{1} \nabla I_{N \lambda} \varepsilon_{2} .
\end{aligned}
$$

Again, we could replace the left hand side by $\left(\epsilon_{2}, \nabla Q\right)^{2}$. The desired estimate follows from the same manipulation as for (1). Finally, (3) follows from orthogonality condition imposed by $\epsilon$ and the small deformation of $Q$ to $Q_{b}$.

Claim 5:

$$
\begin{aligned}
& \left|\left(\epsilon_{1}, \widetilde{L}\right)\right|+\left|\left(\epsilon_{2}, \widetilde{K}\right)\right|+\left|\left(\widetilde{\epsilon}_{1}, \Lambda\left(Q^{\frac{4}{d}} \operatorname{Re} \widetilde{\zeta}_{b}\right)\right)\right|+\left|\left(\widetilde{\epsilon}_{2}, \Lambda\left(Q^{\frac{4}{d}} \operatorname{Im} \widetilde{\zeta}_{b}\right)\right)\right| \\
\leq & \delta\left(\alpha^{*}\right)\left(\int\left|\nabla I_{N \lambda} \tilde{\epsilon}^{2}+\int\right| \widetilde{\epsilon}^{2} e^{-|y|}\right)+\Gamma_{b}^{1+z_{0}} .
\end{aligned}
$$

Proof:

Using the expression of $\widetilde{L}$, the property of $Q_{b}, \widetilde{\zeta}_{b}$, we estimate

$$
\begin{aligned}
\left|\left(\epsilon_{1}, \widetilde{L}\right)\right| & =\left|\int \epsilon_{1}\left[\left(\left(\frac{4 \Sigma^{2}}{d\left|Q_{b}\right|^{2}}+1\right)\left|Q_{b}\right|^{\frac{4}{d}}-\left(1+\frac{4}{d}\right) Q^{\frac{4}{d}}\right) \Lambda \operatorname{Re} \widetilde{\zeta}_{b}+\frac{4 \Sigma \Theta}{d\left|Q_{b}\right|^{2}}\left|Q_{b}\right|^{\frac{4}{d}} \Lambda \operatorname{Im} \widetilde{\zeta}_{b}\right]\right| \\
& \leq C\left(\int|\epsilon|^{2} e^{-\frac{2}{d}(1-C \eta) \frac{\theta(b|y|)}{|b|}}\right)^{\frac{1}{2}} \Gamma_{b}^{1+\frac{C}{d}-C \eta} \\
& \leq \delta\left(\alpha^{*}\right)\left(\int\left|\nabla I_{N \lambda} \widetilde{\epsilon}\right|^{2}+\int \mid \widetilde{\epsilon}^{2} e^{-|y|}\right)+\Gamma_{b}^{1+z_{0}}
\end{aligned}
$$

Similarly for $\left|\left(\epsilon_{2}, \widetilde{K}\right)\right|$. For the last two terms, we estimate, for example

$$
\begin{aligned}
\left|\left(\widetilde{\epsilon}_{1}, \Lambda\left(Q^{\frac{4}{d}} \operatorname{Re} \widetilde{\zeta}_{b}\right)\right)\right| & \leq \int \mid \widetilde{\epsilon}\left(\left|\widetilde{\zeta}_{b}\right|+\left|\nabla \widetilde{\zeta}_{b}\right|\right) e^{-\kappa|y|} \\
& \leq\left(\int \mid \widetilde{\epsilon}^{2} e^{-\kappa|y|}\right)^{\frac{1}{2}} \Gamma_{b}^{\frac{1}{2}+z_{0}^{\prime}} \\
& =\left(\Gamma_{b}^{z_{0}^{\prime}} \int|\widetilde{\epsilon}|^{2} e^{-\kappa|y|}\right)^{\frac{1}{2}} \Gamma_{b}^{\frac{1+z_{0}^{\prime}}{2}} \\
& \leq \delta\left(\alpha^{*}\right)\left(\int\left|\nabla I_{N \lambda} \widetilde{\epsilon}\right|^{2}+\int \mid \widetilde{\epsilon}^{2} e^{-|y|}\right)+\Gamma_{b}^{1+z_{0}}
\end{aligned}
$$

Step 5: Estimate of principal terms.

Claim 6:

$$
\begin{aligned}
& H\left(I_{N \lambda} \epsilon-\widetilde{\zeta}_{b}, I_{N \lambda} \epsilon-\widetilde{\zeta}_{b}\right)-\frac{\left(\widetilde{\epsilon_{1}}, L_{+} \Lambda^{2} Q\right)\left(\widetilde{\epsilon_{1}}, \Lambda Q\right)}{\|\Lambda Q\|_{L^{2}}^{2}}-\left[\left(\operatorname{Re} \widetilde{\zeta}_{b}, \Lambda \operatorname{Re} F\right)+\left(\operatorname{Im} \widetilde{\zeta}_{b}, \Lambda \operatorname{Im} F\right)\right] \\
\geq & c_{1}\left(\int\left|\nabla I_{N \lambda} \widetilde{\epsilon}\right|^{2}+\int \mid \widetilde{\epsilon}^{2} e^{-|y|}+\Gamma_{b}\right)-\delta\left(\alpha^{*}\right) \Xi(s)
\end{aligned}
$$

Proof:

Indeed, we may replace $I_{N \lambda}-\widetilde{\zeta}_{b}$ by $I_{N \lambda} \widetilde{\epsilon}$ by adding an error $\Gamma_{b}^{1+z_{0}}$ which does not 
change the type of the desired estimate. From the spectral property and Claim 4, we have

$$
\begin{aligned}
& H\left(I_{N \lambda} \widetilde{\epsilon}, I_{N \lambda} \widetilde{\epsilon}\right)-\frac{\left(\widetilde{\epsilon_{1}}, L_{+} \Lambda^{2} Q\right)\left(\widetilde{\epsilon_{1}}, \Lambda Q\right)}{\|\Lambda Q\|_{L^{2}}^{2}} \geq c_{0}\left(\int\left|I_{N \lambda} \widetilde{\epsilon}\right|^{2}+\int\left|I_{N \lambda} \widetilde{\epsilon}\right|^{2} e^{-|y|}\right) \\
- & \frac{1}{c_{0}}\left[\left(\widetilde{\epsilon}_{1}, Q\right)^{2}+\left(\widetilde{\epsilon}_{1},|y|^{2} Q\right)^{2}+\left(\widetilde{\epsilon}_{1}, y Q\right)^{2}+\left(\widetilde{\epsilon}_{2}, \Lambda Q\right)^{2}+\left(\widetilde{\epsilon}_{2}, \Lambda^{2} Q\right)^{2}+\left(\widetilde{\epsilon}_{2}, \nabla Q\right)^{2}\right] \\
\geq & c_{1}\left(\int\left|I_{N \lambda} \widetilde{\epsilon}\right|^{2}+\int|\widetilde{\epsilon}|^{2} e^{-|y|}\right)-\delta\left(\alpha^{*}\right) \Xi(s)-\Gamma_{b}^{1+z_{0}}
\end{aligned}
$$

Finally, we conclude by the estimate proved in [20]:

$$
-\left[\left(\operatorname{Re} \widetilde{\zeta}_{b}, \Lambda \operatorname{Re} F\right)+\left(\operatorname{Im} \widetilde{\zeta}_{b}, \Lambda \operatorname{Im} F\right)\right]>c \Gamma_{b}
$$

Step 6: Estimate of original reminder terms and conclusion.

The classical remainder term $\mathcal{R}$ emainder has been already estimated in the proof of Lemma 3.4, while the term $\Upsilon_{N \lambda}$ can be also bounded easily by $\Gamma_{b}^{1+z_{0}}$. Combining Step 1 to Step 5, we obtain the desired estimate.

Next, we need to control the $L^{2}$ type of term $\int_{A}^{2 A}|\varepsilon|^{2}$ in (3.24). This is achieved by computing the flux of $L^{2}$ norm escaping the radiative zone. To do it, we introduce a radial nonnegative cutoff function $\psi(r)$ such that

$$
\psi(r)=\left\{\begin{array}{ll}
0 & r \leq \frac{1}{2} \\
1 & r \geq 3,
\end{array} \quad \frac{1}{4} \leq \psi^{\prime}(r) \leq \frac{1}{2} \quad \text { for } 1 \leq r \leq 2, \psi^{\prime}(r) \geq 0 .\right.
$$

Let

$$
\psi_{A}(s, r)=\psi\left(\frac{r}{A(s)}\right),
$$

with $A(s)$ being given by (3.22), and so

$$
\left\{\begin{array}{l}
\psi_{A}(r)=0 \text { for } 0 \leq r \leq \frac{A}{2} \\
\frac{1}{4 A} \leq \psi_{A}^{\prime}(r) \leq \frac{1}{2 A} \text { for } A \leq r \leq 2 A \\
\psi_{A}(r)=1 \text { for } r \geq 3 r \\
\psi_{A}^{\prime}(r) \geq 0,0 \leq \psi_{A}(r) \leq 1
\end{array}\right.
$$

Moreover, we restrict the freedom on the choice of the parameters $(\eta, a)$ by assuming $a>C \eta$.

Lemma 3.9 ( $L^{2}$ dispersion at infinity in space). There holds for some universal constants $C, c_{3}>0$ and s large enough:

$$
\left\{\int \psi_{A}|\varepsilon|^{2}\right\}_{s} \geq c_{3} b \int_{A}^{2 A}|\varepsilon|^{2}-\frac{C}{b^{2}} \Xi(s)-\Gamma_{b}^{1+C a}-\Gamma_{b}^{\frac{a}{2}} \int\left|\nabla I_{N(s) \lambda(s)} \varepsilon\right|^{2} .
$$

Proof. Take the inner product of (3.3) with $\psi_{A} \varepsilon_{1}$ and of (3.4) with $\psi_{A} \varepsilon_{2}$ and integrate by parts. Note that the supports of $\left(Q_{b}, \Psi_{b}\right)$ and $\psi_{A}$ are disjoint. Then, we obtain

$$
\begin{aligned}
\frac{1}{2}\left\{\int \psi_{A}|\varepsilon|^{2}\right\}_{s}= & \frac{1}{2} \int \frac{\partial \psi_{A}}{\partial s}|\varepsilon|^{2}+\frac{b}{2} \int y \cdot \psi_{A}|\varepsilon|^{2}+\operatorname{Im}\left(\int \nabla \psi_{A} \cdot \nabla \varepsilon \bar{\varepsilon}\right) \\
& -\frac{1}{2}\left(\frac{\lambda_{s}}{\lambda}+b\right) \int y \cdot \nabla \psi_{A}|\varepsilon|^{2}-\frac{1}{2} \frac{x_{s}}{\lambda} \int \nabla \psi_{A}|\varepsilon|^{2}
\end{aligned}
$$


First observe from the choice of $\psi$ :

$$
10 \int \psi^{\prime}\left(\frac{y}{A}\right)|\varepsilon|^{2} \geq \frac{1}{A} \int y \cdot \nabla \psi\left(\frac{y}{A}\right)|\varepsilon|^{2} \geq \frac{1}{10} \int \psi^{\prime}\left(\frac{y}{A}\right)|\varepsilon|^{2} \geq \frac{1}{40} \int_{A}^{2 A}|\varepsilon|^{2} .
$$

The main term is

$$
\frac{b}{2} \int y \cdot \psi_{A}|\varepsilon|^{2} \geq \frac{1}{20} \int \psi^{\prime}\left(\frac{y}{A}\right)|\varepsilon|^{2} .
$$

Using (3.22) and (3.19), we get

$$
\begin{gathered}
\int \frac{\partial \psi_{A}}{\partial s}|\varepsilon|^{2}=-\frac{A_{s}}{A^{2}} \int y \cdot \nabla \psi\left(\frac{y}{A}\right)|\varepsilon|^{2}=2 a \frac{b_{s}}{A b^{2}} \int y \cdot \nabla \psi\left(\frac{y}{A}\right)|\varepsilon|^{2} \\
\geq \frac{a}{A b^{2}}\left[c_{0}\left(\Xi(s)+\int\left|\nabla I_{N(s) \lambda(s)} \varepsilon(s)\right|^{2}+\int|\varepsilon(s)|^{2} e^{-|y|}\right)-\Gamma_{b(s)}^{1-C \eta}\right] \int \psi^{\prime}\left(\frac{y}{A}\right)|\varepsilon|^{2} .
\end{gathered}
$$

Next, by Cauchy-Schwartz inequality, we have

$$
\begin{aligned}
& \left|\int \nabla \psi_{A} \cdot \nabla \varepsilon \bar{\varepsilon}\right| \\
\leq & \left|\int \nabla \psi_{A} \cdot \nabla\left(I_{N \lambda} \varepsilon\right) \bar{\varepsilon}\right|+\left|\int \nabla \psi_{A} \cdot \nabla\left(\left(\operatorname{Id}-I_{N \lambda}\right) \varepsilon\right) \bar{\varepsilon}\right| \\
\leq & \frac{b}{100} \int \psi^{\prime}\left(\frac{y}{A}\right)|\varepsilon|^{2}+\Gamma_{b}^{\frac{a}{2}} \int\left|\nabla I_{N \lambda} \varepsilon\right|^{2}+\left|\int \nabla \psi_{A} \cdot \nabla\left(\left(\operatorname{Id}-I_{N \lambda}\right) \varepsilon\right) \bar{\varepsilon}\right| .
\end{aligned}
$$

On the other hand, by duality, Bernstein's inequaltiy, and interpolation, we estimate

$$
\begin{aligned}
\left|\int \nabla \psi_{A} \cdot \nabla\left(\left(\mathrm{Id}-I_{N \lambda}\right) \varepsilon\right) \bar{\varepsilon}\right| & \leq\left\|\left(\mathrm{Id}-I_{N \lambda}\right) \varepsilon\right\|_{\dot{H}^{\frac{1}{2}}}\left\|\nabla \psi_{A} \bar{\varepsilon}\right\|_{\dot{H}^{\frac{1}{2}}} \\
& \lesssim \frac{(N \lambda)^{\frac{1}{2}-s}}{A}\|\varepsilon\|_{\dot{H}^{s}}\|\varepsilon\|_{\dot{H}^{\frac{1}{2}}} \\
& \lesssim \Gamma_{b}^{\frac{a}{2}} \lambda(t)^{\frac{1-\beta}{\beta}\left(s-\frac{1}{2}\right)}\|\varepsilon\|_{L^{2}}^{1-\frac{1}{2 s}}\left\|I_{N \lambda} \varepsilon\right\|_{\dot{H}^{1}}^{1+\frac{1}{2 s}} \\
& \leq \Gamma_{b}^{10} .
\end{aligned}
$$

Using (3.20), we derive

$$
\left.\left.\left|\frac{x_{s}}{\lambda} \int \nabla \psi_{A}\right| \varepsilon\right|^{2}\left|\leq \frac{C}{A} \int \psi^{\prime}\left(\frac{y}{A}\right)\right| \varepsilon\right|^{2} \leq \Gamma_{b}^{\frac{a}{2}} \int \psi^{\prime}\left(\frac{y}{A}\right)|\varepsilon|^{2} .
$$

By the same way, we have by (3.19)

$$
\begin{aligned}
& \left.\left|\left(\frac{\lambda_{s}}{\lambda}+b\right) \int y \cdot \nabla \psi_{A}\right| \varepsilon\right|^{2} \mid \\
\leq & C\left(\Xi(s)+\int\left|\nabla I_{N(s) \lambda(s)} \varepsilon(s)\right|^{2}+\int|\varepsilon(s)|^{2} e^{-|y|}+\Gamma_{b(s)}^{1-C \eta}\right) \int \psi^{\prime}\left(\frac{y}{A}\right)|\varepsilon|^{2} .
\end{aligned}
$$

Corollary 3.10 (Lyapounov functional). For some universal constant $C>0$ anf for s large, the following holds:

$$
\begin{aligned}
\{\mathcal{J}\}_{s} \leq & -C b\left(\Gamma_{b}+\Xi(s)+\int\left|\nabla I_{N(s) \lambda(s)} \tilde{\varepsilon}\right|^{2}+\int|\tilde{\varepsilon}(s)|^{2} e^{-|y|}+\int_{A}^{2 A}|\varepsilon(s)|^{2}\right) \\
& +C \frac{\Xi(s)}{b^{2}},
\end{aligned}
$$


with

$$
\begin{aligned}
\mathcal{J}(s)= & \left(\int\left|Q_{b}\right|^{2}-\int Q^{2}\right)+2\left(\varepsilon_{1}, \Sigma\right)+2\left(\varepsilon_{2}, \Theta\right)+\int\left(1-\psi_{A}\right)|\varepsilon|^{2} \\
& -c\left(b \tilde{f}_{1}(b)-\int_{0}^{b} \tilde{f}_{1}(v) d v+b\left[\operatorname{Re}\left(\varepsilon_{2}, \Lambda \tilde{\zeta}\right)-\operatorname{Im}\left(\varepsilon_{1}, \Lambda \tilde{\zeta}\right)\right]\right)
\end{aligned}
$$

where $c>0$ denotes some small enough universal constant and

$$
\tilde{f}_{1}(b)=\frac{b}{4}\left\|y Q_{b}\right\|_{L^{2}}^{2}+\frac{1}{2} \operatorname{Im}\left(\int(y \cdot \nabla \tilde{\zeta}) \overline{\tilde{\zeta}}\right) .
$$

Finally, by the same argument as Subsection 4.3 in [5], we conclude the proof of the bootstrap Lemma 1.13

3.3. End proof of Proposition 1.9. Now, we are in position to proving Proposition [1.9. The proof is the same as in [5]. The main difference is the step 4: Strong $L^{2}$ convergence of excess mass outside the blowup point and Step 5: nonconcentration of the $L^{2}$ norm at the blowup point. We are reduced to show the following lemma.

Lemma 3.11. Let $R>0$. Let $x(T)=\lim _{t \rightarrow T} x(t)$. Then, there exists $u^{*} \in L^{2}$ such that

$$
u(t) \rightarrow u^{*} \text { in } L^{2}\left(\mathbb{R}^{d} \backslash\{|x-x(T)| \leq R\}\right) \text { as } t \rightarrow T .
$$

Furthermore, there holds

$$
\int\left|u^{*}\right|^{2}=\lim _{t \rightarrow T} \int \chi_{R(t)}\left|I_{N(t)} u(t)\right|^{2}, R(t)=10 A(t) \lambda(t),
$$

with

$$
\chi_{R}(x)=\chi\left(\frac{x-x(t)}{R}\right), \chi(x)= \begin{cases}0 & |x| \leq 1 \\ 1 & |x| \geq 2 .\end{cases}
$$

Proof. First, we prove the claim. For $R>0$, let $w_{R}(t, x)=\chi_{R}(x)\left[I_{N(t)} u(t, x)\right]$ then, $w_{R}$ solves

$$
i \partial_{t} w_{R}+\Delta w_{R}=i \chi_{R} \tilde{I}_{N} u+2 \nabla \chi_{R} \cdot \nabla I_{N} u+\Delta \chi_{R} I_{N} u-\chi_{R} I_{N}\left(|u|^{\frac{4}{d}} u\right) .
$$

where

$$
\widehat{\tilde{I}_{N} u}(\xi)=-\frac{N_{t}}{N} \tilde{m}_{N}(\xi) \widehat{I_{N} u}(\xi), \tilde{m}_{N}(\xi)=\frac{\xi}{N} \cdot \frac{\nabla m_{N}(\xi)}{m_{N}(\xi)} .
$$

It follows from [5] that

$$
\left\|\int_{0}^{t} e^{i(t-\tau) \Delta}\left(i \chi_{R} \tilde{I}_{N} u+2 \nabla \chi_{R} \cdot \nabla I_{N} u+\Delta \chi_{R} I_{N} u\right)(\tau) d \tau\right\|_{L_{t}^{\infty} L_{x}^{2}\left([0, T] \times \mathbb{R}^{d}\right)}<+\infty .
$$

We only need to estimate the nonlinear term

$$
\left\|\int_{0}^{t} e^{i(t-\tau) \Delta} \chi_{R} I_{N}\left(|u|^{\frac{4}{d}} u\right) d \tau\right\|_{L_{t}^{\infty} L_{x}^{2}\left([0, T] \times \mathbb{R}^{d}\right)}<+\infty .
$$

And

$$
\begin{aligned}
& \left\|\int_{0}^{t} e^{i(t-\tau) \Delta} \chi_{R} I_{N}\left(|u|^{\frac{4}{d}} u\right) d \tau\right\|_{L_{t}^{\infty} L_{x}^{2}\left([0, T] \times \mathbb{R}^{d}\right)} \leq C\left\|\chi_{R} I_{N}\left(|u|^{\frac{4}{d}} u\right)\right\|_{L_{t}^{2} L_{x}^{\frac{2 d}{d+2}}\left([0, T] \times \mathbb{R}^{d}\right)} \\
\lesssim & \left\|\chi_{R}\left(\left|I_{N} u\right|^{\frac{4}{d}} I_{N} u\right)\right\|_{L_{t}^{2} L_{x}^{\frac{2 d}{d+2}}\left([0, T] \times \mathbb{R}^{d}\right)}+\left\|\left|I_{N} u\right|^{\frac{4}{d}} I_{N} u-I_{N}\left(|u|^{\frac{4}{d}} u\right)\right\|_{L_{t}^{2} L_{x}^{\frac{2 d}{d+2}}\left([0, T] \times \mathbb{R}^{d}\right)} .
\end{aligned}
$$


On one hand,

$$
\begin{aligned}
\left\|\chi_{R}\left(\left|I_{N} u\right|^{\frac{4}{d}} I_{N} u\right)\right\|_{L_{t}^{2} L_{x}^{\frac{2 d}{d+2}}\left([0, T] \times \mathbb{R}^{d}\right)}^{2} & =\int_{0}^{T}\left\|\chi_{R}^{\frac{d}{d+4}} I_{N} u\right\|_{\frac{L_{x}}{\frac{2(d+4)}{d+2}}}^{\frac{2(d+4)}{d}} d t \\
& \lesssim \int_{0}^{T}\left\|\chi_{\frac{R}{2}} I_{N} u\right\|_{L_{x}^{\frac{2(d+4)}{d+2}}}^{\frac{2(d+4)}{d+2}} d t \\
& \lesssim \int_{0}^{T}\left\|\nabla\left(\chi_{\frac{R}{2}} I_{N} u\right)\right\|_{L^{2}}^{2}<+\infty,
\end{aligned}
$$

where the last inequality follows from (4.49) in [5]. On the other hand, we have by Lemma 2.13 and (2.32)

$$
\begin{aligned}
& \left\|\left|I_{N} u\right|^{\frac{4}{d}} I_{N} u-I_{N}\left(|u|^{\frac{4}{d}} u\right)\right\|_{L_{t}^{2} L_{x}^{\frac{2 d}{d+2}}\left([0, T] \times \mathbb{R}^{d}\right)}^{2} \\
\lesssim & \sum_{k=k_{0}}^{+\infty} \sum_{j=1}^{J_{k}}\left\|\left|I_{N} u\right|^{\frac{4}{d}} I_{N} u-I_{N}\left(|u|^{\frac{4}{d}} u\right)\right\|_{L_{t}^{2} L_{x}^{\frac{2 d}{d+2}}\left(\left[\tau_{k}^{j}, \tau_{k}^{j+1}\right] \times \mathbb{R}^{d}\right)}^{2} \\
\lesssim & \sum_{k=k_{0}}^{+\infty} \sum_{j=1}^{J_{k}} \lambda\left(t_{k}\right)^{\frac{2}{\beta}} \min \left\{1, \frac{4}{d}\right\} s_{-} \frac{8}{d} \\
\lesssim & \sum_{k=k_{0}}^{+\infty} k \lambda\left(t_{k}\right)^{\frac{2}{\beta}\left(\min \left\{1, \frac{4}{d}\right\} s_{-} \frac{1-s}{s}\right)-\frac{8}{d}} \\
\lesssim & \sum_{k=k_{0}}^{+\infty} k 2^{-\frac{2 k}{\beta}\left(\min \left\{1, \frac{4}{d}\right\} s_{-} \frac{1-s}{s}\right)+\frac{8 k}{d}}<+\infty
\end{aligned}
$$

provided that $\frac{1}{\beta}>\frac{4 s}{\min \{4, d\} s^{2}-(1-s)}$. Then, by the standard argument as in [5], we obtain (3.39).

Next, we turn to show (3.40). Observer that $I_{N} u$ solves

$$
i \partial_{t}\left(I_{N} u\right)+\Delta\left(I_{N} u\right)=i \tilde{I}_{N} u-I_{N}\left(|u|^{\frac{4}{d}} u\right) .
$$

We then compute the flux of $L^{2}$-norm:

$$
\begin{aligned}
& \frac{1}{2} \frac{d}{d \tau} \int \chi\left(\frac{x-x(\tau)}{R(t)}\right)\left|I_{N} u(\tau)\right|^{2} d x \\
= & \frac{1}{R(t)} \operatorname{Im}\left(\int \nabla \chi\left(\frac{x-x(\tau)}{R(t)}\right) \cdot \nabla I_{N} u \overline{I_{N} u}\right)-\frac{x_{t}}{2 R(t)} \cdot \int \nabla \chi\left(\frac{x-x(\tau)}{R(t)}\right)\left|I_{N} u(\tau)\right|^{2} \\
& +\operatorname{Re}\left(\int \chi\left(\frac{x-x(\tau)}{R(t)}\right) \tilde{I}_{N} u \overline{I_{N} u}\right)+\operatorname{Im}\left(\chi\left(\frac{x-x(\tau)}{R(t)}\right)\left(I_{N} u\left|I_{N} u\right|^{\frac{4}{d}}-I_{N}\left(u|u|^{\frac{4}{d}}\right)\right) \overline{I_{N} u}\right)
\end{aligned}
$$

and integrate from $t \rightarrow T$. We obtain

$$
\begin{aligned}
& \left.\quad\left|\int \chi\left(\frac{x-x(T)}{R(t)}\right)\right| u^{*}\right|^{2}-\int \chi\left(\frac{x-x(t)}{R(t)}\right)\left|I_{N} u(t)\right|^{2} \mid \\
& \lesssim \frac{1}{A(t) \lambda(t)} \int_{t}^{T}\left\|\nabla I_{N(\tau)} u(\tau)\right\|_{L^{2}} d \tau+\frac{1}{A(t) \lambda(t)} \int_{t}^{T}\left|\frac{x_{s}}{\lambda}\right| \frac{d \tau}{\lambda(\tau)}+\int_{t}^{T}\left|\int \chi\left(\frac{x-x(\tau)}{R(\tau)}\right) \tilde{I}_{N} u \overline{I_{N} u}\right| \\
& \quad+\int_{t}^{T} \int\left|\left(I_{N} u\left|I_{N} u\right|^{\frac{4}{d}}-I_{N}\left(u|u|^{\frac{4}{d}}\right)\right) \overline{I_{N} u}\right|
\end{aligned}
$$


By the same argument as in [5], we obtain

$$
\begin{aligned}
& \frac{1}{A(t) \lambda(t)} \int_{t}^{T}\left\|\nabla I_{N(\tau)} u(\tau)\right\|_{L^{2}} d \tau+\frac{1}{A(t) \lambda(t)} \int_{t}^{T}\left|\frac{x_{s}}{\lambda}\right| \frac{d \tau}{\lambda(\tau)}+\int_{t}^{T}\left|\int \chi\left(\frac{x-x(\tau)}{R(\tau)}\right) \tilde{I}_{N} u \overline{I_{N} u}\right| \\
& \rightarrow 0 \quad \text { as } \quad t \rightarrow T .
\end{aligned}
$$

Hence, we only need to show

$$
\int_{t}^{T} \int\left|\left(I_{N} u\left|I_{N} u\right|^{\frac{4}{d}}-I_{N}\left(u|u|^{\frac{4}{d}}\right)\right) \overline{I_{N} u}\right| \rightarrow 0 \quad \text { as } \quad t \rightarrow T
$$

Indeed, by (2.29), we obtain

$$
\begin{aligned}
& \int_{t}^{T} \int\left|\left(I_{N} u\left|I_{N} u\right|^{\frac{4}{d}}-I_{N}\left(u|u|^{\frac{4}{d}}\right)\right) \overline{I_{N} u}\right| \\
\lesssim & \sum_{k=k_{t}}^{+\infty} \sum_{j=1}^{J_{k}}\left\|I_{N} u\right\|_{L_{t}^{2} L_{x}^{\frac{2 d}{d-2}}\left(\left[\tau_{k}^{j}, \tau_{k}^{j+1}\right] \times \mathbb{R}^{d}\right)}\left\|I_{N} u\left|I_{N} u\right|^{\frac{4}{d}}-I_{N}\left(|u|^{\frac{4}{d}} u\right)\right\|_{L_{t}^{2} L_{x}^{\frac{2 d}{d+2}}\left(\left[\tau_{k}^{j}, \tau_{k}^{j+1}\right] \times \mathbb{R}^{d}\right)} \\
\lesssim & \sum_{k=k_{t}}^{+\infty} k \lambda\left(t_{k}\right)^{\frac{1}{\beta}\left(\min \left\{1, \frac{4}{d}\right\} s-\frac{1-s}{s}\right)-\min \left\{1, \frac{4}{d}\right\}} \\
\lesssim & \sum_{k=k_{t}}^{+\infty} k 2^{-k\left(\frac{1}{\beta}\left(\min \left\{1, \frac{4}{d}\right\} s-\frac{1-s}{s}\right)-\min \left\{1, \frac{4}{d}\right\}\right)} \rightarrow 0 \quad \text { as } \quad k_{t} \rightarrow+\infty
\end{aligned}
$$

Acknowledgements. J. Zheng was supported by NSFC Grants 11771041, 11831004. Part of this work was completed while J. Zheng was supported by the the European Research Council, ERC-2014- CoG, project number 646650 Singwave at Université de Nice.

\section{REFERENCES}

[1] H. Berestycki, P. L. Lions, Nonlinear scalar field equations. I Existence of a ground state, Arch. Rational Mech. Anal. 82:4 (1983), 313-345.

[2] T. Cazenave and F. Weissler, The Cauchy problem for the critical nonlinear Schrödinger equation in $H^{s}$. Nonlinear Anal., 14(1990), 807-836.

[3] M. Christ and M. Weinstein, Dispersion of small amplitude solutions of the generalized Korteweg-de Vries equation. J. Funct. Anal., 100(1991), 87-109.

[4] J. Colliander, M. Grillakis and N. Tzirakis, Tensor products and correlation estimates with applications to nonlinear Schrödinger equations. Comm. Pure and Applied Math., 62 (2009), 920-968.

[5] J. Colliander and P. Raphael, Rough blowup solutions to the $L^{2}$ critical NLS, Math. Anna., 345(2009), 307-366.

[6] B. Dodson, Global well-posedness and scattering for the mass critical nonlinear Schrödinger equation with mass below the mass of the ground state. Adv. Math., 285(2015), 1589-1618.

[7] G. Fibich, F. Merle and P. Raphael, Proof of a Spectral Property related to the singularity formation for the $L^{2}$ critical nonlinear Schrödinger equation. Physica D, 220(2006), 1-13.

[8] B. Gidas, W.M. Ni, L. Nirenberg, Symmetry and related properties via the maximum principle, Comm. Math. Phys. 68 (1979), 209-243.

[9] J. Ginibre and G. Velo, On a class of nonlinear Schrödinger equations. I. The Cauchy problem, general case. J. Funct. Anal. 32(1979), 1-32.

[10] J. Ginibre and G. Velo, Time decay of finite energy solutions of the nonlinear Klein-Gordon and Schrödinger equations, Ann. Inst. H. Poincaré Phys. Théor. 43 (1985), 399-442.

[11] M. Keel and T. Tao, Endpoint Strichartz estimates. Amer. J. Math. 120:5 (1998), 955-980. 
[12] R. Killip, T. Tao, and M. Visan, The cubic nonlinear Schrödinger equation in two dimensions with radial data. J. Eur. Math. Soc., 11 (2009), 1203-1258.

[13] R. Killip, M. Visan, and X. Zhang, The mass-critical nonlinear Schrödinger equation with radial data in dimensions three and higher. Analysis and Partial Differential Equations, 1, no. 2 (2008) 229-266.

[14] M.K. Kwong, Uniqueness of positive solutions of $\Delta u-u+u^{p}=0$ in $\mathbb{R}^{n}$, Arch. Rational Mech. Anal. 105:3 (1989), 243-266.

[15] F. Merle, On uniqueness and continuation properties after blow-up time of self-similar solutions of nonlinear Schrödinger equation with critical exponent and critical mass , Comm. Pure Appl. Math. 45 (1992), no. 2, 203-254.

[16] F. Merle, Determination of blow-up solutions with minimal mass for nonlinear Schrödinger equations with critical power. Duke Math. J., 69(1993), 427-454.

[17] F. Merle and P. Raphael, The blow-up dynamic and upper bound on the blow-up rate for critical nonlinear Schrödinger equation. Ann. Math., 161(2005), 157-222.

[18] F. Merle and P. Raphael, Sharp upper bound on the blow-up rate for the critical nonlinear Schrödinger equation. Geom. Funct. Anal., 13(2003) 591-642.

[19] F. Merle and P. Raphael, On universality of blow-up profile for $L^{2}$ critical nonlinear Schrödinger equation. Invent. math. 156(2004), 565-672.

[20] F. Merle and P. Raphael, On a sharp lower bound on the blow-up rate for the $L^{2}$ critical nonlinear Schrödinger equation. J. AMS, 19(2005), 37-90.

[21] F. Merle and P. Raphael, Profiles and Quantization of the Blow Up Mass for Critical Nonlinear Schrödinger Equation. Commun. Math. Phys., 253(2005), 675-704.

[22] F. Merle and P. Raphael, On one blow-up point solutions to the critical nonlinear Schrödinger equation. Jour. of Hype. Diff. Equa., 2(2005), 919-962.

[23] F. Merle and P. Raphael, Blow up of the critical norm for some radial $L^{2}$ super critical nonlinear Schrödinger equations, Amer. J. Math. 130 (2008), 945-978.

[24] F. Merle, P. Raphael and J. Szeftel, The instability of Bourgain-Wang solutions for the $L^{2}$ critical NLS, Amer. Jour. Math., 135(2013), 967-1017.

[25] P. Raphael, Stability of the log-log bound for blow-up solutions to the critical nonlinear Schrödinger equation. Math. Ann., 331(2005), 577-609.

[26] E. C. Titchmarsh, Eigenfunction expansions associated with second-order differential equations, Oxford, Clarendon Press, 1946.

[27] M. Visan and X. Zhang, On the blowup for the $L^{2}$-critical focusing nonlinear Schrödinger equation in higher dimensions below the energy class. SIAM J. Math. Anal., 39(2007), 34-56.

[28] M. I. Weinstein, Nonlinear Schrödinger equations and sharp interpolation estimates, Comm. Math. Phys., 87(1983), 567-576.

[29] M.I. Weinstein, Modulational stability of ground states of nonlinear Schrödinger equations, SIAM J. Math. Anal., 16(1985), 567-576.

[30] K. Yang, S. Roudenko and Y. Zhao Blow-up dynamics and spectral property in the $L^{2}$-critical nonlinear Schrödinger equation in high dimensions, arXiv:1712.07647.

Université Côte D'Azur, CNRS, LJAD, France

E-mail address: csun@unice.fr

Institute of Applied Physics and Computational Mathematics, Beijing 100088

E-mail address: zhengjiqiang@gmail.com 